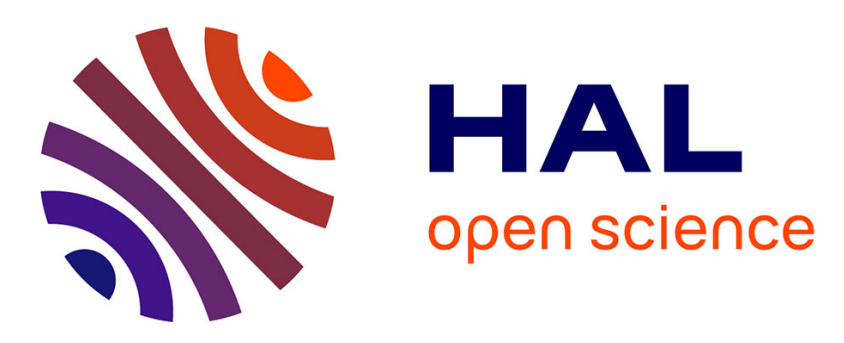

\title{
Stability constraints for oceanic numerical models: implications for the formulation of time and space discretizations
}

Florian Lemarié, Laurent Debreu, Gurvan Madec, Jérémie Demange, Jean-Marc Molines, Marc Honnorat

\section{To cite this version:}

Florian Lemarié, Laurent Debreu, Gurvan Madec, Jérémie Demange, Jean-Marc Molines, et al.. Stability constraints for oceanic numerical models: implications for the formulation of time and space discretizations. Ocean Modelling, 2015, 92, pp.124-148. 10.1016/j.ocemod.2015.06.006 . hal01065979v2

\section{HAL Id: hal-01065979 \\ https://hal.inria.fr/hal-01065979v2}

Submitted on 26 Jun 2015

HAL is a multi-disciplinary open access archive for the deposit and dissemination of scientific research documents, whether they are published or not. The documents may come from teaching and research institutions in France or abroad, or from public or private research centers.
L'archive ouverte pluridisciplinaire HAL, est destinée au dépôt et à la diffusion de documents scientifiques de niveau recherche, publiés ou non, émanant des établissements d'enseignement et de recherche français ou étrangers, des laboratoires publics ou privés. 


\title{
Stability Constraints for Oceanic Numerical Models: Implications for the Formulation of Time and Space Discretizations
}

\author{
F. Lemarié ${ }^{\mathrm{a}, \mathrm{b}, \mathrm{c}, *}$, L. Debreu $^{\mathrm{a}, \mathrm{b}, \mathrm{c}}$, G. Madec $^{\mathrm{d}, \mathrm{f}, \mathrm{b}, \mathrm{c}, \mathrm{a}}$, J. Demange $^{\mathrm{a}, \mathrm{b}, \mathrm{c}}$, \\ J.M. Molines ${ }^{\mathrm{e}}$, M. Honnorat ${ }^{\mathrm{a}, \mathrm{b}, \mathrm{c}}$ \\ ${ }^{\mathrm{a}}$ Inria \\ ${ }^{\mathrm{b}}$ Univ. Grenoble Alpes, LJK, F-38000 Grenoble, France \\ ${ }^{\mathrm{c}} \mathrm{CNRS}$, LJK, F-38000 Grenoble, France \\ ${ }^{\mathrm{d}}$ Sorbonne Universités (UPMC, Univ Paris 06)-CNRS-IRD-MNHN, LOCEAN Laboratory, \\ Paris, France \\ ${ }^{\mathrm{e}}$ CNRS-Université Grenoble Alpes, Laboratoire de Glaciologie et Géophysique de \\ l'Environnement, Grenoble, France \\ ${ }^{\mathrm{f}}$ National Oceanography Centre, Southampton, UK
}

\begin{abstract}
Except for vertical diffusion (and possibly the external mode and bottom drag), oceanic models usually rely on explicit time-stepping algorithms subject to Courant-FriedrichsLewy (CFL) stability criteria. Implicit methods could be unconditionally stable, but an algebraic system must be solved at each time step and other considerations such as accuracy and efficiency are less straightforward to achieve. Depending on the target application, the process limiting the maximum allowed time-step is generally different. In this paper, we introduce offline diagnostics to predict stability limits associated with internal gravity waves, advection, diffusion, and rotation. This suite of diagnostics is applied to a set of global, regional and coastal numerical simulations with several horizontal/vertical resolutions and different numerical models. We show that, for resolutions finer that $1 / 2^{\circ}$, models with an eulerian vertical coordinate are generally constrained by vertical advection in a few hot spots and that numerics must be extremely robust to changes in Courant number. Based on those results, we review the stability and accuracy of existing numerical kernels in vogue in primitive equations oceanic models with a focus on advective processes and the dynamics of internal waves. We emphasize the additional value of studying the numerical kernel of oceanic models in the light of coupled space-time approaches instead of studying the time schemes independently from spatial discretizations. From this study, we suggest some guidelines for the development of temporal schemes in future generation multi-purpose oceanic models.
\end{abstract}


Key words: Space-time discretization schemes, Von-Neumann stability analysis, Advection schemes, Internal gravity waves

\section{Introduction}

\subsection{Context}

Owing to advances in computational power, global climate models are now configured with increasingly higher horizontal/vertical resolution. The extension of the range of application of this type of model, originally developed for low-resolution large-scale configurations, raises some new challenges of numerical and physical nature (Griffies, 2013; Griffies and Treguier, 2013) and requires the accurate representation of a wider energy spectrum. Moreover, multi-resolution configurations via 1-way or 2-way nesting techniques are now mature (Debreu and Blayo, 2008; Debreu et al., 2012) and could be used to locally reach marginal submesoscales resolving resolutions (e.g. Marchesiello et al., 2011). Low-resolution configurations imply relatively slow and laminar (linear) flows while many emerging issues arise when extending the range to finer scales: the appropriate representation of internal wave dynamics is increasingly important to predict physical mixing (e.g. Arbic et al., 2012), the addition of biogeochemical tracers imposes new constraints on advection schemes (e.g. Lévy et al., 2001), adequate capture of the transfers between resolved and unresolved scales is required (Thuburn et al., 2014), spurious dianeutral mixing remains a key issue in the presence of mesoscale eddies (Ilicak et al., 2012). An other difficulty is the proper synergy between physical paramerizations and the numerics to ensure regularity of the physical solutions by limiting fine-scale variance (e.g. Hecht, 2010). More generally, high-resolution modeling requires a finer consideration of numerical methods. This has motivated the emergence of comprehensive initiatives like the COMODO ${ }^{1}$ and DCMIP ${ }^{2}$ projects aimed at intercomparison of numerical kernels to exploring the merits of different approaches through a suite of semi-idealized testcases.

The objective of this paper is to define the main guidelines for the development of temporal schemes in future generation multi-purpose (i.e. used for applications ranging from paleo-climate to eddying regimes) oceanic models. Because changing the time-stepping algorithm is one of the most fundamental change to an existing numerical code, it is legitimate to first proceed to a deep investigation of the requirements. Originally, for climate models, temporal discretizations were mainly chosen for their simplicity and upon additional criteria :

(i) their ability to conserve quadratic quantities (e.g. energy, potential vorticity or enstrophy) when combined with specifically designed second-order centered schemes for non-linear momentum advective terms formulated in a vector invariant form of the shallow-water equations (e.g. Sadourny, 1975). This led to the choice of the Leapfrog scheme in the momentum equation to minimize dissipation.

\footnotetext{
* Corresponding author.

Phone: $+33(0) 476514860$

Fax: +33(0)4 76631263

E-mail address: florian.lemariedinria.fr.

1 French Numerical Ocean Modeling Community http://www. comodo-ocean. fr

2 Dynamical Core Model Intercomparison Project https://www.earthsystemcog.org/projects/ dcmip/
} 
Energy and/or potential enstrophy conserving schemes are considered an efficient way to suppress nonlinear instabilities.

(ii) conservation of the second moments (i.e. quadratic variance) for tracers which resulted in the use of centered-in-space and Leapfrog-in-time schemes for tracer advection.

(iii) for their computational efficiency (i.e. the stability range with respect to the number of computations of the rhs) for internal gravity waves. An efficient way for extending the stability range of Leapfrog based models is the pressure gradient averaging approach of Brown and Campana (1978) which is still being used in several numerical models (Griffies et al., 2000).

As a result, most numerical models were historically based on a leapfrog scheme for both the tracer and momentum equations, combined with second-order centered schemes. Note that, even if most models now favor the flux form to the vector-invariant form of momentum equations, it remains crucial to know as accurately as possible the discrete properties of numerical schemes in terms of energy dissipation to close the energy cycle (Eden et al., 2014).

When high-resolution turbulent regimes start emerging in numerical simulations the algorithmic choices must evolve beyond the Leapfrog-in-time - second-order in space framework to accommodate to new contraints (e.g. Shchepetkin and McWilliams, 2005). Indeed, as mentioned above, other important properties arise for finer resolution: accuracy, for correct shape preservation and phase speed, preservation of positivity hence good stability for advective and diffusive processes, good dissipation properties when highfrequency forcings are used, etc. This is especially true for terms integrated using explicit time-stepping but also for terms integrated using implicit methods (typically vertical/isoneutral diffusion and bottom drag) for which accuracy considerations should not be overlooked. In most existing numerical models, the space-time algorithms are derived by studying separately the space and time dimensions assuming the other dimension is continuous (i.e. the underlying partial differential equation is semi-discretized, e.g. Hundsdorfer and Trompert, 1994; Shchepetkin and McWilliams, 2005). There is, however, a second standard reasoning to derive space-time algorithms: the coupled space-time approach for which both space and time dimensions are discretized (e.g. Lax and Wendroff, 1960; Leonard, 1979; Daru and Tenaud, 2004). This approach is more tedious to use but is expected to provide a more accurate measure of the stability and numerical errors for a given sub-system of the full system of equations under consideration ${ }^{3}$. Indeed, this would serve little purpose to combine a low-order temporal scheme with a high-order space discretization. A clear advantage of the space-time approach is to combine errors associated with the space and time discretizations in the same study.

A long-standing belief is that a given process when integrated using small Courant numbers (compared to the CFL constraint) has inherently small numerical errors associated to the time-stepping algorithm. From this perspective, improving the order of accuracy of the time-stepping algorithm would not be a priority as long as sufficiently small time-steps are chosen. This statement is, however, inexact because it ignores the interaction between time and space discretization errors. It is not unusual to see space-time discretization schemes with large numerical errors for small Courant numbers and less errors close to their stability limit (e.g. a Leapfrog scheme combined with a second-order centered scheme). The objective of the present study is first to estimate the typical order of magnitude of Courant numbers encountered in realistic configurations. Then, using those estimates, we will proceed to a deep investigation of the behavior of space-time numerical schemes usually found in state-of-the-art oceanic models over a relevant range of Courant numbers. In this case, numerical schemes will be studied close to their functioning

3 The coupled space-time approach is only viable when applied to selected terms in the equations; e.g. advection, gravity waves or Coriolis considered separately. It is not applicable to the primitive equations as a whole. 


\begin{tabular}{|c|c|c|c|}
\hline \multicolumn{4}{|l|}{ State variables } \\
\hline$q$ & $\begin{array}{l}\text { Three dimensional tracer (can be temperature or salin- } \\
\text { ity) }\end{array}$ & & \\
\hline $\mathbf{u}=(u, v, w)$ & Three dimensional velocity field & & \\
\hline \multicolumn{4}{|l|}{ Acronyms } \\
\hline $\mathrm{LF}$ & Leapfrog & $\mathrm{C} 2$ & Second-order centered scheme \\
\hline $\mathrm{AM}$ & Adams-Moulton & UP3 & Third-order upwind scheme \\
\hline RA & Robert-Asselin filter & QK3 & Quickest scheme \\
\hline $\mathrm{AB}$ & Adams-Bashforth & LW & Lax-Wendroff scheme \\
\hline RK & Runge-Kutta & $\mathrm{Co} 4$ & Fourth-order compact scheme \\
\hline UP1 & First-order upwind scheme & Co4st & Space-time fourth-order compact scheme \\
\hline CTU & Corner-transport upwind scheme & FCT & Flux-Corrected Transport \\
\hline \multicolumn{4}{|c|}{ Coordinates and spatial operators } \\
\hline$x, y$ & Horizontal coordinates & $\Delta x, \Delta y, \Delta z$ & Measures of the grid-box interfaces $[\mathrm{m}]$ \\
\hline$z$ & Vertical coordinate & $\Delta t$ & Time-step for the temporal discretization $[\mathrm{s}]$ \\
\hline \multicolumn{4}{|l|}{ Parameters } \\
\hline$c_{1}$ & Phase speed of the first baroclinic mode $\left[\mathrm{m} \mathrm{s}^{-1}\right]$ & $k$ & Spatial wavenumber in $x$ direction $\left[\mathrm{m}^{-1}\right]$ \\
\hline$\alpha_{\text {igw }}$ & $\begin{array}{l}\text { Courant number for internal gravity waves propaga- } \\
\text { tion }\end{array}$ & $\theta$ & Normalized wavenumber, $k \Delta x$ \\
\hline$\alpha_{\mathrm{adv}}$ & Advective Courant number & $\omega$ & Complex damping coefficient $\left[\mathrm{s}^{-1}\right]$ \\
\hline$\alpha_{\text {igw }}^{\star}$ & CFL criterion for internal gravity waves propagation & $\mu$ & Damping coefficient normalized by $\Delta t(\mu=\omega \Delta t)$ \\
\hline$\alpha_{\mathrm{adv}}^{\star}$ & CFL criterion for advection & $\mu_{i}$ & Imaginary part of $\mu$ \\
\hline$\varepsilon$ & Stabilizing parameter for the AB2 scheme & $\mu_{r}$ & Real part of $\mu$ \\
\hline$\nu$ & Robert-Asselin filter parameter & & \\
\hline
\end{tabular}

Important notations used throughout the paper (including appendices)

conditions found during realistic simulations.

A first step toward our objective is to determine which terms are expected to be the most important in terms of stability for a given target application. Using a scaling analysis, Griffies and Adcroft (2008) studied the time step constraints introduced by the Coriolis term, advection, internal gravity wave propagation, as well as biharmonic viscosity and harmonic diffusion with respect to the horizontal grid spacing. Their study suggests that the details of the numerical integration schemes must be considered to get a clear estimate of which process sets the time-step. For the typical resolution of today's climate models it is unlikely that inertial oscillations or the viscous/diffusive operators will be responsible for the time-step limitation. We, thus, focus our study on three-dimensional advection and internal gravity waves, assuming that the numerical models under consideration are discretized on a horizontal C-grid with an Eulerian vertical coordinate in the primitive equations limit. Moreover, we assume that sea-ice or external gravity waves (through the "leakage" of the barotropic mode (e.g. Griffies et al., 2000; Shchepetkin and McWilliams, 2005; Demange et al., 2014b) ) do not contribute to the limitation of the maximum allowed time-step. Important notations used throughout the paper are summarized in Tab. 1.

\subsection{Stability of Numerical Models}

Throughout this paper, $x$ and $y$ are the horizontal directions aligned with the computational grid, $z$ is the vertical coordinate oriented upward from the topography $-h_{i, j}$ to the free surface $\zeta_{i, j}$. We note 
$\mathbf{u}=(u, v, w)$ the three-dimensional velocity, $w$ being the dia-surface velocity, and $\mathcal{V}$ the volume of a given grid cell

$$
\mathcal{V}_{i, j, k}=\Delta x_{i, j, k} \Delta y_{i, j, k} \Delta z_{i, j, k}
$$

To formulate the stability constraint associated with three-dimensional advection, we need to define the volumetric fluxes $U_{i+\frac{1}{2}, j, k}, V_{i, j+\frac{1}{2}, k}$ and $W_{i, j, k+\frac{1}{2}}$, respectively in the two horizontal and vertical direction. Those fluxes are the velocities multiplied by the area of the corresponding grid cell face, i.e.

$$
\left\{\begin{aligned}
U_{i+\frac{1}{2}, j, k} & =\Delta z_{i+\frac{1}{2}, j, k} \Delta y_{i+\frac{1}{2}, j} u_{i+\frac{1}{2}, j, k} \\
V_{i, j+\frac{1}{2}, k} & =\Delta z_{i, j+\frac{1}{2}, k} \Delta x_{i, j+\frac{1}{2}} v_{i, j+\frac{1}{2}, k} \\
W_{i, j, k+\frac{1}{2}} & =\Delta x_{i, j} \Delta y_{i, j} w_{i, j, k+\frac{1}{2}}
\end{aligned}\right.
$$

Using those notations, the horizontal Courant numbers $\alpha_{\mathrm{adv}}^{x}$ in the $x$-direction and $\alpha_{\mathrm{adv}}^{y}$ in the $y$-direction are given by

$$
\begin{aligned}
& \left(\alpha_{\mathrm{adv}}^{x}\right)_{i, j, k}=\Delta t \cdot\left[\max \left\{U_{i+\frac{1}{2}, j, k}, 0\right\}-\min \left\{U_{i-\frac{1}{2}, j, k}, 0\right\}\right] / \mathcal{V}_{i, j, k} \\
& \left(\alpha_{\mathrm{adv}}^{y}\right)_{i, j, k}=\Delta t \cdot\left[\max \left\{V_{i, j+\frac{1}{2}, k}, 0\right\}-\min \left\{V_{i, j-\frac{1}{2}, k}, 0\right\}\right] / \mathcal{V}_{i, j, k}
\end{aligned}
$$

and the vertical one by

$$
\left(\alpha_{\text {adv }}^{z}\right)_{i, j, k}=\Delta t \cdot\left[\max \left\{W_{i, j, k+\frac{1}{2}}, 0\right\}-\min \left\{W_{i, j, k-\frac{1}{2}}, 0\right\}\right] / \mathcal{V}_{i, j, k}
$$

Those Courant numbers are positive definite and they are defined in a finite-volume sense to be consistent with the discretization paradigm used in numerical models (e.g. Shchepetkin and McWilliams, 2005; Griffies and Adcroft, 2008; Griffies and Treguier, 2013). It is straightforward to see that the quantity $\left[\alpha_{\mathrm{adv}}^{x}+\alpha_{\mathrm{adv}}^{y}+\alpha_{\mathrm{adv}}^{z}\right]_{i, j, k}$ is the fraction of water volume transported into the adjacent grid cells during one time step. The stability condition due to three-dimensional advection generally reads

$$
\left(\alpha_{\mathrm{adv}}^{x}\right)_{i, j, k}+\left(\alpha_{\mathrm{adv}}^{y}\right)_{i, j, k}+\beta\left(\alpha_{\mathrm{adv}}^{z}\right)_{i, j, k} \leq \alpha_{\mathrm{adv}}^{\star} .
$$

with $\alpha_{\mathrm{adv}}^{\star}$ the advective Courant-Friedrichs-Lewy (CFL) stability criterion, and $\beta$ a coefficient arising from the fact that different advection schemes with different stability criteria may be used in the horizontal and vertical directions. The meaning of $\beta$ will be clarified later in Sec. 2.4. For some specific discretization schemes, the Courant numbers in each spatial direction are no longer additive, as in (1.3), but independent (i.e. the multidimensional scheme is stable as long as the 1D scheme is stable in each spatial direction), such examples are given in Sec. 2.4. Note that the Courant numbers (1.1-1.2) could also be defined in a centered way, however we found it more natural to adopt an upstream approach because in the remainder of the paper we mainly consider third-order spatial discretizations and we do not expect major changes in our conclusions depending on the way to discretize the Courant number.

The derivation of the stability condition for internal gravity waves is less straightforward to obtain. It requires an expansion of the primitive equations in terms of baroclinic modes $M_{m}(z)$ to retrieve the speed of the fastest internal wave. The baroclinic modes $M_{m}(z)$ are defined by 


$$
\left\{\begin{aligned}
\Lambda M_{m}(z) & =c_{m}^{-2} M_{m}(z) & & z \in]-h, 0[ \\
\frac{d M_{m}}{d z}(-h) & =0 & & m=0,1,2, \ldots \\
M_{m}(0) & =-g N^{-2}(0) \frac{d M_{m}}{d z}(0) & &
\end{aligned}\right.
$$

with $\Lambda=-\frac{d}{d z}\left(N^{-2}(z) \frac{d}{d z}\right)$, and $N(z)$ the Brunt-Väisälä frequency. Because $\Lambda$ is a compact symmetric operator, it admits a basis of orthonormal eigenvectors $M_{m}(z)$ with positive eigenvalues $c_{m}^{-2} \cdot c_{m}$ is the gravity wave speed associated with $m$ th baroclinic mode. By convention, $c_{m}$ 's are sorted in decreasing order, so that $m=0$ corresponds to the barotropic mode, and $m=1$ to the first baroclinic mode whose associated wave speed $c_{1}$ is the quantity of interest to study the stability of numerical models because it corresponds to the speed of the fastest internal wave. The various steps and assumptions necessary to separate the inviscid primitive equations into baroclinic modes can be found in Blayo and Debreu (2005) (Sec. 4.2.2) and later in Sec. 2.3. It is straightforward to show that the stability condition for a two-dimensional wave equation with constant wave speed $c_{1}$ is

$$
\Delta t \sqrt{\left(c_{1}\right)_{i, j}^{2}\left(\frac{1}{\left(\Delta x_{i, j}\right)^{2}}+\frac{1}{\left(\Delta y_{i, j}\right)^{2}}\right)} \leq \alpha_{\mathrm{igw}}^{\star}
$$

with $\alpha_{\text {igw }}^{\star}$ the CFL stability criterion associated with the space-time discretization used for internal waves. In (1.5), the computation of $c_{1}$ at each water column $(i, j)$ requires the solution of the boundary value problem (1.4). Note that even if (1.5) does not involve the vertical discretization, the latter is taken into account in the computation of the hydrostatic balance used to derive the operator $\Lambda$, hence the phase speed $c_{1}$. However, since we are only interested in $c_{1}$, we expect a rather weak sensitivity to the vertical discretization and to the number of vertical levels because the first baroclinic mode is well resolved even at low vertical resolution.

This paper is organized as follows. Estimates of the typical values of $\alpha_{\mathrm{adv}}^{\star}$ and $\alpha_{\mathrm{igw}}^{\star}$ representative of the state-of-the-art oceanic models are given in Sec. 2 using a coupled space-time stability analysis. Thanks to this analysis, we derive in Sec. 3 practical stability limitations of several different numerical models run at different horizontal/vertical resolution. Accuracy for processes subject to a time-implicit treatment is also considered. Finally, in Sec. 4 we summarize our findings and provide the implications for the design of space and time algorithms in next-generation numerical models.

\section{Space-Time stability analysis of some numerical schemes used in existing models}

Most of the numerical schemes routinely used in oceanic models are generally based on separate time and space discretizations in contrast with a coupled space-time approach (e.g. Lax and Wendroff, 1960; Leonard, 1979; Daru and Tenaud, 2004), an exception is tracer advection in the MIT General Circulation Model (Ilicak et al., 2012; Adcroft et al., 2014). The stability and accuracy analysis of these decoupled space-time approach (sometimes referred to as method of lines (e.g. Hundsdorfer and Trompert, 1994)) are generally done considering time schemes with perfect spatial discretizations, i.e. by considering the Fourier image $i k$ of ideal derivative (e.g. Shchepetkin and McWilliams, 2005). This approach is very useful to study separately the effect of time stepping but the stability limit thus obtained can be very different from the actual stability limit when the effect of the spatial discretization is accounted for (i.e. when 
$i k$ is replaced by the Fourier image of the advection scheme under consideration). Indeed, the increase of the order of accuracy of spatial schemes leads to a decrease in stability limit. An example of such interactions between space and time discretizations is the Leapfrog (LF) scheme combined with a secondorder centered spatial discretization : phase-lead errors due to the LF scheme cancel exactly with the phasedelay due to the spatial differencing to provide an exact space-time scheme for $\alpha_{\mathrm{adv}}^{\star}=1$. Methodologies for space-time analysis are given in Daru and Tenaud (2004) or Baldauf (2008). In this section we first briefly describe some delicacies associated to the design of a numerical kernel, then the stability results obtained for discretization methods generally found in state-of-the-art oceanic models are presented in the light of a coupled space-time analysis.

\subsection{Synergetics of numerical methods}

The numerics for primitive equations oceanic models is generally designed by studying separately subsystems describing the different physical processes at play among advection, diffusion, external or internal pure gravity waves propagation and inertia. It is a convenient way to proceed because the requirement can be rather different depending on the process under consideration. For example, external gravity waves can be integrated with a time-stepping different from the rest of the code, vertical diffusion and viscosity can be solved implicitly with a backward Euler scheme. The situation is however more complicated for other terms. Because rotation, internal gravity waves and advection are strongly connected, the possibility to use totally different time-stepping for those terms would lead to splitting errors which would be hard to diagnose. The ultimate goal is thus to be able to optimize stability, accuracy, and efficiency simultaneously for each of those processes. However, mutual compatibility and interferences of methods makes this task very arduous, e.g. sometimes time-stepping coefficients optimized for gravity waves are not the best for advection and Coriolis, and inversely (e.g. Shchepetkin and McWilliams, 2005). Depending on the target applications, stability and accuracy are more or less crucial for a given term, compromises must be done. The difficulty is that a numerical kernel must be designed as a whole, not just as an advection or a wave propagation equation. Descriptions of such interferences between numerical methods are rarely reported in the literature, examples are given in Beckers and Deleersnijder (1993) or Walters et al. (2009). Among other things, those studies emphasize the fact that the numerics for pure gravity waves must be chosen under the constraint to be stable for the Coriolis term. Because this paper is dedicated to the study of existing numerical methods we do not directly face this difficulty. However this necessary synergy between numerical methods used to integrate the different terms in the equations is crucial when designing a numerical kernel.

\subsection{Inertial oscillations}

In the remainder of the paper we focus on numerical methods for advection and pure gravity waves propagation because we anticipate that the Coriolis force does no longer limit the time-step at the resolution typically used in climate models, with the exception of paleo-climate type studies. We briefly remind that the equations of free motion on a rotating plane are

$$
\left\{\begin{array}{l}
\partial_{t} u=f v \\
\partial_{t} v=-f u
\end{array}\right.
$$


where $f$ is the Coriolis parameter. Note that if $f v$ and $f u$ are discretized at the same moment in time, (2.1) can be numerically studied as an advection equation for the variable $\mathscr{U}=u+i v$. To mimic the properties of the continuous problem, the discretization of (2.1) is required to have no contribution to the kinetic energy budget. On a C-grid this property can only be satisfied globally and not locally. Equations (2.1) yield to a stability constraint of the form $f \Delta t \leq \alpha_{\text {cor }}^{\star}$, where $\alpha_{\text {cor }}^{\star}$ depends on the time-stepping algorithm. The maximum value of $1 / f$ is $\frac{12 \mathrm{~h}}{2 \pi} \approx 6875 \mathrm{~s}$ at the north pole. Typical values for $\alpha_{\text {cor }}^{\star}$ are between 0.75 and 1.5 which sets the maximum allowed time-step for a climate model around $\Delta t=5400 \mathrm{~s}$. This value is significantly larger than the time-step used in practice for most global models at a resolution of $1 / 2^{\circ}$ and higher. We, thus, assume that the time-step of eddying ocean models is too short for Coriolis to affect our analysis, hence we investigate in more details advection and gravity waves propagation to know which process limits the time-step.

\subsection{Internal gravity waves}

The usual way to study the integration of pure internal gravity waves is to work in the space of baroclinic modes. We first recall here the different steps of this decomposition starting from the $2 \mathrm{D} x-\mathrm{z}$ inviscid primitive equations without rotation and linearized around $\mathbf{u}=\left(u_{0}, v_{0}, 0\right)$

$$
\left\{\begin{aligned}
\partial_{t} u+u_{0} \partial_{x} u+\frac{1}{\rho_{0}} \partial_{x} p & =0 \\
\partial_{z} p & =-\rho g \\
\partial_{x} u+\partial_{z} w & =0 \\
\partial_{t} \rho+u_{0} \partial_{x} \rho+w \frac{d \bar{\rho}}{d z} & =0
\end{aligned}\right.
$$

where $p$ and $\rho$ are pressure and density perturbations around an hydrostatically balanced reference state $(\bar{p}(z), \bar{\rho}(z))$ with $\frac{d \bar{p}}{d z}=-\bar{\rho} g$. In (2.2), the different equations respectively represent momentum conservation, hydrostatic equilibrium, continuity/incompressibility, and tracer conservation (assuming a linear equation of state). Boundary conditions are $w(z=-h)=0$ and $p(z=0)=\rho_{0} g \zeta$. Let us note that the phase speed of internal gravity waves does not appear explicitly in the primitive equations. To exhibit this phase speed we consider a state of rest $\left(u_{0}, v_{0}\right)=(0,0)$ and a simple forward-backward scheme. In this case, the time-integration of (2.2) reads

(i) compute pressure using the hydrostatic relation

$$
p^{n}=g \int_{z}^{0} \rho^{n} d z^{\prime}+\rho_{0} g \zeta
$$

(ii) advance momentum

$$
u^{n+1}=u^{n}-\frac{\Delta t}{\rho_{0}} \partial_{x} p^{n}
$$

(iii) compute vertical velocity from continuity equation

$$
w^{n+1}=-\int_{-h}^{z} \partial_{x} u^{n+1} d z^{\prime}
$$

(iv) advance density

$$
\rho^{n+1}=\rho^{n}-\Delta t w^{n+1} \frac{d \bar{\rho}}{d z}
$$


Next step is to project the equations onto the baroclinic modes. On a discrete vertical grid with $n_{z}$ vertical levels for the discretization, the solution of this semi-discrete set of equations can be sought under the form

$$
\left\{\begin{array}{l}
u^{n}(x, z)=\sum_{q=0}^{n_{z}-1} u_{q}^{n}(x) M_{q}(z) \\
p^{n}(x, z)=\rho_{0} g \sum_{q=0}^{n_{z}-1} p_{q}^{n}(x) M_{q}(z) \\
\rho^{n}(x, z)=-\rho_{0} \sum_{q=0}^{n_{z}-1} p_{q}^{n}(x) \frac{d M_{q}}{d z}(z)
\end{array}\right.
$$

where $M_{q}(z)$ is the solution of Sturm-Liouville problem (1.4) with $N^{2}(z)=-\frac{g}{\rho_{0}} \frac{d \bar{\rho}}{d z}$ the square of the Brunt-Väisälä frequency. Note that $p^{n}(x, z)$ and $\rho^{n}(x, z)$ in this form satisfy the hydrostatic relation. Substitution of (2.3) into the semi-discrete equations for the forward-backward scheme and assuming a flatbottom leads to the following momentum equation for each mode $m$

$$
u_{m}^{n+1}=u_{m}^{n}-g \Delta t \partial_{x} p_{m}^{n}(x)
$$

thanks to the orthonormality condition $\frac{1}{h} \int_{h}^{0} M_{q}(z) M_{m}(z) d z=\delta_{m q}, \forall m, q \geq 1$ where $\delta_{m q}$ is the conventional Kronecker delta. This relationship comes from the fact that the eigenvectors $M_{m}$ form an orthonormal basis. We also get

$$
w^{n+1}=\underbrace{-\sum_{q=0}^{n_{z}-1} \partial_{x} u_{q}^{n+1} \int_{-h}^{z} M_{q}\left(z^{\prime}\right) d z^{\prime}}_{\text {continuity equation }}=\underbrace{g N^{-2}(z) \sum_{q=0}^{n_{z}-1}\left(\frac{p_{q}^{n+1}-p_{q}^{n}}{\Delta t}\right) \frac{d M_{q}}{d z}}_{\text {tracer conservation }} .
$$

Using (1.4) we get that $N^{-2}(z) \frac{d M_{q}}{d z}=-c_{q}^{-2} \int_{-h}^{z} M_{q}\left(z^{\prime}\right) d z^{\prime}$, and (2.5) thus simplifies to

$$
\partial_{x} u_{m}^{n+1}=-g c_{m}^{-2} \frac{p_{m}^{n+1}-p_{m}^{n}}{\Delta t}
$$

again using the orthonormality condition. The forward-backward approach applied to gravity waves propagation can thus be simply written as

$$
\left\{\begin{array}{l}
\frac{u_{m}^{n+1}-u_{m}^{n}}{\Delta t}=-g \partial_{x} p_{m}^{n} \\
\frac{p_{m}^{n+1}-p_{m}^{n}}{\Delta t}=-\frac{c_{m}^{2}}{g} \partial_{x} u_{m}^{n+1}
\end{array}\right.
$$

It is straightforward to see that $\partial_{x} p_{m}^{n}$ is the horizontal pressure gradient and $\partial_{x} u_{m}^{n+1}$ the horizontal divergence in the continuity equation. To our knowledge, all numerical models use second-order centered schemes to discretize those two terms. Moreover the time-stepping for $u_{m}$ (resp. $p_{m}$ ) is equivalent to the one used for momentum (resp. tracers) in the three-dimensional code. The forward-backward scheme for internal waves is stable if (2.7) is stable for all $m$.

For the sake of simplicity the subscript $m$ is dropped in the remainder of the paper. Moreover, we assume that the phase speed $c$ is the one of the first baroclinic vertical mode (i.e. the baroclinic mode with 
faster phase speed). Changing $p_{m}$ in $\frac{c_{m}}{g} p_{m}$, the continuous counterpart of (2.7) is

$$
\left\{\begin{array}{l}
\partial_{t} u+c \partial_{x} p=0 \\
\partial_{t} p+c \partial_{x} u=0 .
\end{array}\right.
$$

The numerical study of the full system (2.2) can thus be done using a simpler system of two equations representing the coupling between momentum and pressure/tracers. Discrete stability constraints in both systems are equivalent. It is worth mentioning that the choice $\left(u_{0}, v_{0}\right)=(0,0)$ has two direct implications

(a) the influence of momentum and tracer advection on internal waves dynamics is neglected.

(b) the phase speed in the stability constraint for internal gravity waves is $c_{1}$, the speed associated to the first baroclinic mode, as in (1.5), instead of $c_{1}+\left|\mathbf{u}_{0}\right|$ which amounts to consider that the barotropic component of velocity is significantly smaller than $c_{1}$.

Because a simple forward-backward approach would be unstable in the presence of advective terms (except if coupled space-time schemes are used) it is not used in practice in numerical models. The most popular time-stepping algorithm used in several oceanic climate models (as well as in the barotropic mode of some mesoscale model like $\mathrm{POM}^{4}$ ) for solving (2.8) is the LF scheme with pressure gradient averaging, sometimes referred to as Shuman's averaging (SA) (Brown and Campana, 1978; Griffies et al., 2000), the latter reads

$$
\begin{aligned}
& p_{i}^{n+1, \star}=p_{i}^{n-1}-2 \alpha_{\mathrm{igw}}\left(u_{i+\frac{1}{2}}^{n}-u_{i-\frac{1}{2}}^{n}\right), \\
& u_{i+\frac{1}{2}}^{n+1, \star}=u_{i+\frac{1}{2}}^{n-1}-2 \alpha_{\mathrm{igw}}\left(\left(1-2 \chi_{1}\right)\left(p_{i+1}^{n}-p_{i}^{n}\right)+\chi_{1}\left(p_{i+1}^{n+1, \star}-p_{i}^{n+1, \star}+p_{i+1}^{n-1}-p_{i}^{n-1}\right)\right)
\end{aligned}
$$

with $\alpha_{\text {igw }}=c \Delta t / \Delta x$. In the case of the LF-SA scheme (i.e. $u^{n+1}=u^{n+1, \star}, p^{n+1}=p^{n+1, \star}$ in (2.9)), the stability condition ${ }^{5}$ is $\alpha_{\mathrm{igw}}^{\star}=1$ for $\chi_{1}=1 / 4$. Because of symmetry, same stability limit would be obtained if the averaging is done on $u$ instead of $p$ in (2.9). Note that other choices like the forwardbackward scheme and the time-staggering between $u$ and $p$ (e.g. Griffies, 2010; Adcroft et al., 2014) would lead to the exact same characteristic equation of the LF-SA scheme. For this reason these alternatives are not explicitly discussed here because their properties are equivalent to the ones of the LF-SA scheme.

In Shchepetkin and McWilliams (2005), (2.9) is used as a predictor step followed by the corrector steps

$$
\begin{aligned}
& p_{i}^{n+\frac{1}{2}}=(1 / 2-\gamma) p_{i}^{n+1, \star}+(1 / 2+2 \gamma) p_{i}^{n}-\gamma p_{i}^{n-1} \\
& u_{i+\frac{1}{2}}^{n+\frac{1}{2}}=(1 / 2-\gamma) u_{i+\frac{1}{2}}^{n+1, \star}+(1 / 2+2 \gamma) u_{i+\frac{1}{2}}^{n}-\gamma u_{i+\frac{1}{2}}^{n-1} \\
& p_{i}^{n+1}=p_{i}^{n}-\alpha_{\mathrm{igw}}\left(u_{i+\frac{1}{2}}^{n+\frac{1}{2}}-u_{i-\frac{1}{2}}^{n+\frac{1}{2}}\right) \\
& u_{i+\frac{1}{2}}^{n+1}=u_{i+\frac{1}{2}}^{n}-\alpha_{\mathrm{igw}}\left(\left(1-2 \chi_{2}\right)\left(p_{i+1}^{n+\frac{1}{2}}-p_{i}^{n+\frac{1}{2}}\right)+\chi_{2}\left(p_{i+1}^{n+1}-p_{i}^{n+1}+p_{i+1}^{n}-p_{i}^{n}\right)\right)
\end{aligned}
$$

where proper choice of the parameter $\gamma$ can yield to higher-order accuracy for representing phase speed of internal waves, compared to (2.9). The predictor-corrector scheme (2.9-2.10) is implemented in the

\section{Princeton Ocean Model}

5 In Brown and Campana (1978), a discretization on a collocated A-grid is implicitly assumed, leading to $\alpha_{\text {igw }}^{\star}=2$. It can be shown that this stability condition decreases to $\alpha_{\mathrm{igw}}^{\star}=1$ on a staggered grid (e.g. Sun and Sun, 2011). 
ROMS model. The choice $\left(\gamma, \chi_{1}, \chi_{2}\right)=(1 / 7,1 / 8,1 / 2)$ leads to third-order accuracy in time and large stability limit $\alpha_{\text {igw }}^{\star}=1.183$. However, it would imply that the order of accuracy for advection would drop to 2 and a much reduced stability for advective terms $\left(\alpha_{\mathrm{adv}}^{\star}=0.66\right.$ compared to $\alpha_{\mathrm{adv}}^{\star}=1.587$ for $\gamma=1 / 12$ ). This remark illustrates the compromise that should be made when building a temporal scheme in order to find the best possible combination for accurate representation, good stability properties and ease of implementation of the different terms involved in the equations, as discussed in Sec. 2.1. In the ROMS-UCLA code the combination $\left(\gamma, \chi_{1}, \chi_{2}\right)=(1 / 12,0,1 / 8)$, which leads to $\alpha_{\mathrm{igw}}^{\star}=0.815$, is used while the ROMS-AGRIF code uses $\left(\gamma, \chi_{1}, \chi_{2}\right)=(1 / 12,0,1 / 2)$, which leads to $\alpha_{\text {igw }}^{\star}=0.804$. An other alternative, based on a second-order Runge-Kutta (RK) scheme with forward-backward feedback, is described in Shchepetkin and McWilliams (2008). This scheme has the advantage to have large stability range $\left(\alpha_{\mathrm{igw}}^{\star}=\sqrt{3}=1.732\right)$ and to be free of computational mode. However, as is, it is unstable for centered advection schemes which makes this scheme essentially useful for isopycnic models (indeed, high-order upwind schemes generally used to advect layer thickness are stable with second-order RK).

Based on the schemes previously introduced, we can consider that a value $\alpha_{\mathrm{igw}}^{\star}=1$ is representative of the stability limit for most of the numerical models. Moreover, as long as the spatial order of accuracy for the discretization of the pressure gradient term and the continuity equation is no more than 2 , the benefits of high-order temporal schemes over low-order ones are questionable because both spatial and temporal accuracy should be increased, not just the temporal one. Interestingly enough, a recent study by Demange et al. (2014a) emphasizes that higher-order approximations of both the pressure gradient term and the horizontal divergence in the continuity equation allow a significantly better representation of internal waves propagation, compared to standard algorithmic choices.

\subsection{Stability of Multidimensional Advection}

We consider here the advection equation written in a conservative way along with the incompressibility condition

$$
\left\{\begin{aligned}
\partial_{t} q+\nabla \cdot(\mathbf{u} q) & =0 \\
\nabla \cdot \mathbf{u} & =0 .
\end{aligned}\right.
$$

We retain the continuity equation here to emphasize that the advection equation must be conservative while satisfying a constancy preservation property. In (2.11) $q$ can either represent a tracer or horizontal momentum components when a flux-form of nonlinear terms is used. A very large body of literature is dedicated to the numerical discretization of (2.11) (e.g. Durran, 2010, and references therein). The aim here is not to study all the existing schemes with exhaustivity, but to illustrate the change of behavior we can obtain for the same advection scheme when combined with different temporal discretizations. We consider the third-order upwind scheme (UP3) in the horizontal and the second-order centered (C2) scheme in the vertical as the schemes of reference for this study. We also retain the fourth-order compact scheme (see description in App. B) for vertical advection because it is widely used for regional and coastal models (e.g. Shchepetkin, 2015).

Because all numerical models need some amount of dissipation, it is relevant to consider an oddordered scheme with built-in dissipation in our comparison to ensure that we have the same level of dissipation due to the spatial discretization, whatever the temporal scheme. Moreover, it can be shown that this velocity-dependent dissipation, implicit to the scheme, is naturally tuned to moderate dispersive errors 


\subsubsection{Time-stepping algorithms}

Throughout this paper we consider the following temporal discretizations (see App C for more details)

(i) the Leapfrog scheme with Robert-Asselin filter (LFRA), we note $\nu$ the filter parameter

(ii) the Leapfrog Adams-Moulton predictor-corrector scheme (LFAM3)

(iii) the second-order Adams-Bashforth scheme (AB2 $\varepsilon)$ whose extrapolation in time is biased by $\varepsilon$ to overcome an instability of the scheme

(iv) the third-order Adams-Bashforth scheme (AB3)

(v) the third-order Runge-Kutta (RK3) scheme (as described in Skamarock and Klemp (2008))

(vi) For completeness, we also consider the third-order one-step coupled space-time scheme Euler-QUICKEST (QK3, Leonard, 1979), sometimes referred to as direct scheme (Hundsdorfer and Trompert, 1994; Adcroft et al., 2014). This scheme is an extension of the Lax and Wendroff (1960) scheme to third order accuracy. When QK3 is used in the horizontal we consider the second-order Lax and Wendroff (1960) (LW) scheme in the vertical. Virtually any advection scheme can be used in the vertical without changing the $3 \mathrm{D}$ stability, as long as this scheme is a coupled space-time scheme. For example, a space-time version of the fourth-order compact scheme has been built in App. B for that purpose.

The six options we discuss here are representative of the discretization techniques used in some of the most widely used numerical models, namely the HYbrid Coordinate Ocean Model (HYCOM, Bleck et al., 2002; Wallcraft, 2011), the MIT General Circulation Model (MITGcM, Adcroft et al., 2014), The Modular Ocean Model (MOM4p1, Griffies, 2010), the Nucleus for the European Modeling of the Ocean (NEMO, Madec, 2008), the Parallel Ocean Program (POP, Smith et al., 2010), the Regional Oceanic Modeling System (ROMS, Shchepetkin and McWilliams, 2005), the Princeton Ocean Model (POM) and the atmospheric WRF (Weather Research \& Forecasting) model (Skamarock and Klemp, 2008). The complete description of schemes $(i)$ to $(v i)$ is given in App C.

As shown in Tab. 2, we expect different CFL stability numbers for the UP3 and the C2 discretizations. In this case, we find that a conservative way of expressing the $3 \mathrm{D}$ stability condition (1.3) is

$$
\left(\alpha_{\mathrm{adv}}^{x}\right)_{i, j, k}+\left(\alpha_{\mathrm{adv}}^{y}\right)_{i, j, k}+\beta\left(\alpha_{\mathrm{adv}}^{z}\right)_{i, j, k} \leq \alpha_{\mathrm{up} 3}^{\star}, \quad \beta=\frac{\alpha_{\mathrm{up} 3}^{\star}}{\alpha_{\mathrm{c} 2}^{\star}}
$$

with $\alpha_{\mathrm{up} 3}^{\star}$ and $\alpha_{\mathrm{c} 2}^{\star}$ the CFL numbers respectively associated with the UP3 and C2 spatial discretizations. (2.12) applies to schemes (i) to (iv). For the coupled space-time approach (i.e. QK3 in the horizontal and LW in the vertical), the scheme is extended to multidimensions using a directional splitting procedure (e.g. App. C.2, Leonard et al., 1996; Bott, 2010) which results in the following stability condition

$$
\max \left\{\left(\alpha_{\text {adv }}^{x}\right)_{i, j, k},\left(\alpha_{\text {adv }}^{y}\right)_{i, j, k},\left(\alpha_{\text {adv }}^{z}\right)_{i, j, k}\right\} \leq 1 .
$$

Stability bound (2.13) allows much longer time-steps than (2.12). In practice, a simple conservative-form directional splitting (Strang, 1968) is not sufficient to ensure the property of constancy preservation in (2.11). To circumvent this problem, Leonard et al. (1996) introduced the MACHO procedure, and Bott (2010) an ingenious time-splitting approach (see description in App. C). Those procedures have been already used in the oceanic and atmospheric context (e.g. Duhaut et al., 2008; Yamaguchi et al., 2011). When used for momentum advection, directional splitting can have significant impact on the numerical solutions especially for balanced situations where specific terms in the equations should cancel each other. It also makes it harder to numerically diagnose global budgets for quantities involving both $u$ and $v$ (like 

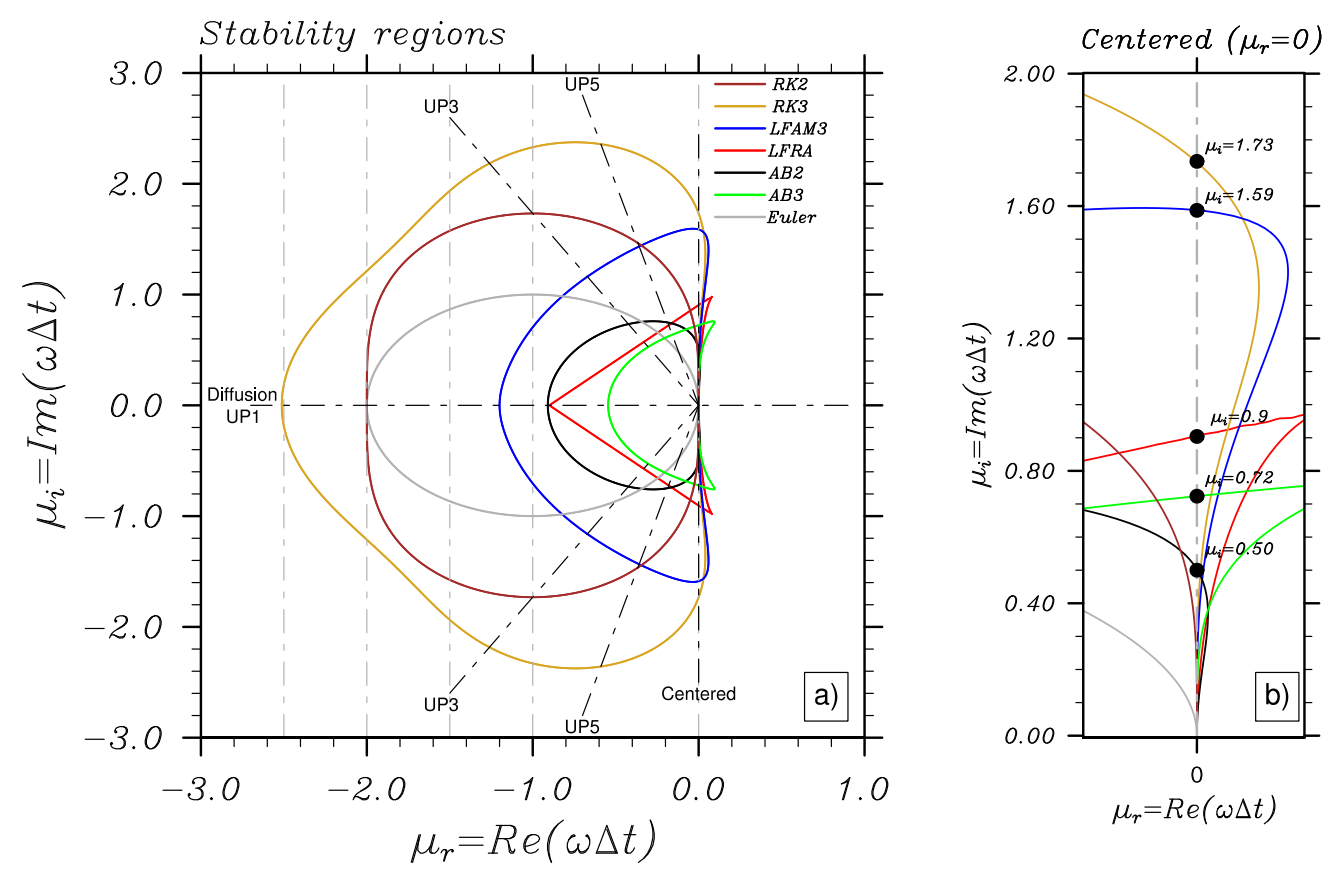

Fig. 1. a) eigenvalue stability boundaries for the second-order Runge Kutta scheme (brown), third-order Runge Kutta scheme (orange), the Leapfrog Adams-Moulton scheme (blue), the Leapfrog scheme with Robert-Asselin filtering $(\nu=0.1)$ (red), the second-order Adams-Bashforth scheme $(\varepsilon=0.1)$ (black), the third-order Adams-Bashforth scheme (green) and the Euler scheme (gray) in the $\left(\mu_{r}, \mu_{i}\right)$ plane with $\mu_{r}$ and $\mu_{i}$ the real and imaginary part of the normalized damping rate $\mu$. The dot-dashed black lines provide directions for different space discretizations. The corresponding space-time discretizations are stable as long as those lines are inside the stability boundaries. b) same as a) with a zoom on the $\mu_{r}=0$ axis (i.e. for purely advective processes). Acronyms are defined in Tab. 1 .

vorticity or kinetic energy budgets).

\subsubsection{Eigenvalue stability regions}

We look at the eigenvalue stability regions for the time stepping algorithms $(i)$ to $(v)$ as well as for the second-order Runge-Kutta scheme (RK2) which, provided that specific parameter tuning is done, may be promising in the context of oceanic models (e.g. Hallberg, 1997; Shchepetkin and McWilliams, 2008). First, the study is done regardless of spatial discretizations. We assume that the tracer $q$ can be Fourier decomposed as

$$
q(x, t)=\sum_{k} \widehat{q}_{k}(t) e^{i k x}, \quad i=\sqrt{-1} .
$$

where $k$ is a wavenumber and $\widehat{q}_{k}$ the amplitude/phase of the corresponding Fourier component. In the case of a combination of advection (first-order derivatives) and diffusion (even order derivatives), substitution of an arbitrary Fourier mode in an advection-diffusion equation would lead to the oscillation equation

$$
\partial_{t} \widehat{q}(t)=-\omega \widehat{q}(t), \quad \omega=\omega_{r}+i \omega_{i},
$$

with $\omega$ a complex coefficient (subscripts $k$ have been dropped for clarity). In (2.15), $\omega_{r}$ determines the change in amplitude and $\omega_{i}$ the change in phase for a given Fourier mode. Time discretization of the $\partial_{t} \widehat{q}$ term in (2.15) with time-step $\Delta t$, using schemes $(i)$ to $(v)$, yields to a general equation 


$$
\widehat{q}^{t+\Delta t}=\mathscr{F}\left(\widehat{q}^{t}, \widehat{q}^{t-\Delta t}\right)-\mu \mathscr{G}\left(\widehat{q}^{t}, \widehat{q}^{t-\Delta t}\right), \quad \mu=\mu_{r}+i \mu_{i}=\omega_{r} \Delta t+i \omega_{i} \Delta t, \quad \widehat{q}^{t}=\widehat{q}(t),
$$

which leads to a second-order characteristic polynomial whose roots are the amplification factor (because this scheme involves $\widehat{q}^{t-2 \Delta t}$, the characteristic polynomial for AB3 is third-order). For each scheme of interest we can easily derive the amplification factor $\lambda$ as a function of $\mu_{r}$ and $\mu_{i}$ :

RK2

RK3

LFAM3

$$
\lambda=1+\mu+\frac{\mu^{2}}{2}
$$

$$
\lambda=1+\mu+\frac{\mu^{2}}{2}+\frac{\mu^{3}}{6},
$$

LFRA

$$
\lambda^{ \pm}=\frac{1}{12}\left(g_{1} \pm \sqrt{\left(-g_{1}\right)^{2}+48 \mu}\right), \quad g_{1}=6+4 \mu+5 \mu^{2},
$$

$\mathrm{AB} 2 \varepsilon$

$$
\lambda^{ \pm}=\nu+i \mu_{i} \pm \sqrt{1-2 \nu+\nu^{2}-\mu_{i}^{2}+2 \mu_{r}},
$$

AB3

$$
\lambda^{ \pm}=\frac{1}{4}\left(g_{2} \pm \sqrt{\left(-g_{2}\right)^{2}-8 \mu(1+2 \varepsilon)}\right), \quad g_{2}=2+(3+2 \varepsilon) \mu .
$$

$$
\lambda \text { is solution of } \quad \lambda^{3}=\left(1+\frac{23}{12} \mu\right) \lambda^{2}-\frac{4}{3} \mu \lambda+\frac{5}{12} \mu
$$

$\mu_{r}=0$ is equivalent to pure advection while $\mu_{i}=0$ is pure diffusion. For schemes involving time level $t-\Delta t$ (i.e. all schemes but RK2 and RK3), eigenvalue $\lambda^{+}$corresponds to a physical mode while $\lambda^{-}$ corresponds to a spurious computational mode. For the LFRA scheme, the amplification factor is derived considering that diffusion is applied at time $n-1$ for stability reasons. For each scheme, the stability boundaries in the $\left(\mu_{r}, \mu_{i}\right)$ plane are located where $\max \left(\left|\lambda^{+}\right|,\left|\lambda^{-}\right|\right)=1$. Stability regions are plotted in Fig. 1. Such simple diagrams provide a substantial amount of information about the behavior of a given scheme.

Let us consider three different cases

- Diffusive processes (i.e. $\mu_{i}=0$ ).

Stability of time-stepping algorithms for diffusion is satisfied when the real axis $\left(\mu_{i}=0\right)$ lies inside the stability boundaries. Critical values $\mu_{r}^{\dagger} \approx-5 / 2$ for RK3, $\mu_{r}^{\dagger}=-2$ for Euler and RK2, $\mu_{r}^{\dagger} \approx-6 / 5$ for LFAM3, $\mu_{r}^{\dagger} \approx-9 / 10$ for $\mathrm{AB} 2 \varepsilon, \mu_{r}^{\dagger} \approx-11 / 20$ for $\mathrm{AB} 3$, and $\mu_{r}^{\dagger} \approx-9 / 10$ for LFRA (i.e. Euler on $2 \Delta t$ with Asselin filtering) can be found from Fig. 1, panel a). Compared to a simple Euler scheme, the Runge-Kutta schemes have good stability properties for diffusion while other schemes are significantly less stable. The values of $\mu_{r}^{\dagger}$ can be directly translated into a more traditional stability constraint. Indeed, for a laplacian operator, it is straightforward to show that $\mu_{r}=-\sigma^{(2)} \mathscr{S}_{\text {Lap }}(\theta)$ with $\sigma^{(2)}$ the parabolic Courant number and $\mathscr{S}_{\text {Lap }}(\theta)=2(1-\cos \theta)$ the Fourier symbol of the laplacian with $\theta=k \Delta x$. Because $\max _{0 \leq \theta \leq \pi} \mathscr{S}_{\text {Lap }}(\theta)=2$, we obtain that the stability boundary corresponds to $\sigma^{(2)} \leq-\mu_{r}^{\dagger} / 4$. We recover the well-known condition $\sigma^{(2)} \leq 1 / 2$ for the Euler scheme. Among third-order accurate schemes, the Runge-Kutta scheme $\left(\sigma^{(2)} \leq 5 / 8\right)$ is twice more stable than LFAM3 $\left(\sigma^{(2)} \leq 3 / 10\right)$ for diffusion, and 5 times more stable than AB3 $\left(\sigma^{(2)} \leq 11 / 80\right.$ ). Same rationale applied to the biharmonic operator (with $\left.\mathscr{S}_{\operatorname{Bih}}(\theta)=16 \sin (\theta / 2)^{4}\right)$ leads to $\sigma^{(4)} \leq-\mu_{r}^{\dagger} / 16$ (with $\sigma^{(4)}=B \Delta t / \Delta x^{4}, B$ the hyper-diffusivity), and same conclusions hold.

- Advective processes (i.e. $\mu_{r}=0$ ).

The values of $\mu_{i}$ at the intersection between the imaginary axis $\left(\mu_{r}=0\right)$ and the stability regions 
provide the stability condition for pure advection. In Fig. 1, panel b) the critical values of $\mu_{i}$ are given. Compared to the diffusive case, the conclusions are significantly different. RK3 and LFAM3 have comparable stability ( $\mu_{i}^{\dagger} \approx 1.73$ versus $\mu_{i}^{\dagger} \approx 1.59$ ), $\mu_{i}^{\dagger} \approx 0.72$ for AB 3 , while among first-order accurate schemes $\operatorname{AB} 2 \varepsilon\left(\mu_{i}^{\dagger}=0.50\right)$ is about 2 times less stable than LFRA $\left(\mu_{i}^{\dagger}=0.904\right)$. Note the different behavior among the Adams-Bashforth schemes: AB $2 \varepsilon$ is significantly more stable than AB3 for pure diffusion while AB3 is more robust for pure advection. It is possible to translate those critical values $\mu_{i}^{\dagger}$ in terms of CFL conditions. For a spatial scheme with Fourier symbol $\mathscr{S}(\theta)$, we get that $\mu_{i}=\alpha_{\text {adv }}|\mathscr{S}(\theta)|$. For linear centered advection schemes we have

$$
\begin{aligned}
& \mathscr{S}_{\mathrm{C} 2}(\theta)=i \sin \theta, \\
& \mathscr{S}_{\mathrm{C} 4}(\theta)=i \frac{8 \sin \theta-\sin 2 \theta}{6} \text {, } \\
& \mathscr{S}_{\mathrm{C} 6}(\theta)=i \frac{45 \sin \theta-9 \sin 2 \theta+\sin 3 \theta}{30}, \\
& \max _{0 \leq \theta \leq \pi}\left|\mathscr{S}_{\mathrm{C} 2}(\theta)\right|=1, \quad \text { for } \quad \theta=\pi / 2 \\
& \max _{0 \leq \theta \leq \pi}\left|\mathscr{S}_{\mathrm{C} 4}(\theta)\right| \approx 1.37222, \quad \text { for } \quad \theta=2 \arctan \sqrt{\frac{3+2 \sqrt{6}}{5}} \\
& \max _{0 \leq \theta \leq \pi}\left|\mathscr{S}_{\mathrm{C} 6}(\theta)\right| \approx 1.58598, \quad \text { for } \quad \theta \approx 1.93607 \text {. }
\end{aligned}
$$

This shows that the critical values $\mu_{i}^{\dagger}$ directly provide the space-time CFL condition when a secondorder centered scheme is used (i.e. stability holds for $\alpha_{\text {adv }} \leq \mu_{i}^{\dagger}$ because $\max _{0 \leq \theta \leq \pi}\left|\mathscr{S}_{\mathrm{C} 2}(\theta)\right|=1$ ). When higher-order advection schemes with better dispersion properties are used, the CFL condition drops whatever the time-stepping algorithm. Indeed, for a fourth-order (resp. sixth-order) advection scheme, the CFL condition is $\alpha_{\text {adv }} \leq \mu_{i}^{\dagger} / 1.37222$ (resp. $\alpha_{\text {adv }} \leq \mu_{i}^{\dagger} / 1.58598$ ). The CFL constraints thus obtained for different combinations of space and time schemes are reported in Tab. 2.

- Upwind schemes (i.e. $\mu_{i} \neq 0, \mu_{r} \neq 0$ ).

The situation is less straightforward to interpret when upwind schemes are used because both $\mu_{r}$ and $\mu_{i}$ are nonzero. In this case, we get that $\mu_{i}=\alpha_{\text {adv }} \Im\{\mathscr{S}(\theta)\}$, and $\mu_{r}=\alpha_{\text {adv }} \Re\{\mathscr{S}(\theta)\}$ thus giving $\mu_{i}=(\Im\{\mathscr{S}(\theta)\} / \Re\{\mathscr{S}(\theta)\}) \cdot \mu_{r}=\varrho(\theta) \cdot \mu_{r}$. For upwind schemes we find that

$$
\begin{array}{llll}
\mathscr{S}_{\mathrm{UP} 1}(\theta)=\frac{\mathscr{S}_{\mathrm{Lap}}(\theta)}{2}+\mathscr{S}_{\mathrm{C} 2}(\theta), & \max _{0 \leq \theta \leq \pi}\left|\mathscr{S}_{\mathrm{UP} 1}(\theta)\right|=2, & \text { for } & \theta=\pi, \\
\mathscr{S}_{\mathrm{UP} 3}(\theta)=\frac{\mathscr{S}_{\mathrm{Bih}}(\theta)}{12}+\mathscr{S}_{\mathrm{C} 4}(\theta), & \max _{0 \leq \theta \leq \pi}\left|\mathscr{S}_{\mathrm{UP} 3}(\theta)\right|=3 / 2, & \text { for } & \theta=2 \pi / 3 .
\end{array}
$$

The CFL constraint for the first-order upwind scheme is easy to find, because $\Im\left\{\mathscr{S}_{\text {UP1 }}(\theta=\pi)\right\}=$ 0 and $\max _{0 \leq \theta \leq \pi}\left|\mathscr{S}_{\mathrm{UP} 1}(\theta)\right|=2$ stability is obtained for $\alpha_{\text {adv }} \leq-\mu_{r}^{\dagger} / 2$ (we recover the condition $\alpha_{\mathrm{adv}} \leq 1$ for the Euler-upwind scheme). For the third-order upwind scheme we find that $\varrho(\theta=2 \pi / 3)=\sqrt{3}$. Assuming that the maximum of the space-time amplification factor $\lambda=$ $\max \left(\left|\lambda^{+}\right|,\left|\lambda^{-}\right|\right)$is obtained when the maximum of $\left|\mathscr{S}_{\mathrm{UP} 3}(\theta)\right|$ is reached, stability with a third-order upwind advection scheme is satisfied as long as the line $\mu_{i}=\sqrt{3} \mu_{r}$ is inside the stability region (Fig. 1 , a). Noting $\left(\mu_{r}^{\ddagger}, \mu_{i}^{\ddagger}\right)$ the coordinates of the intersection between the line $\mu_{i}=\sqrt{3} \mu_{r}$ and the stability boundary of a given scheme, we get that the CFL condition is $\alpha_{\text {adv }} \leq \mu_{r}^{\ddagger} / \Re\{\mathscr{S}(\theta=2 \pi / 3)\} \equiv$ $4 \mu_{r}^{\ddagger} / 3$. From Fig. 1, panel a), we see that the value of $\mu_{r}^{\ddagger}$ is around 1.22 for RK3 and around 0.66 for LFAM3, indicating a much larger stability range for the RK3-UP3 scheme compared to LFAM3-UP3. The fact that the $\mathrm{AB} 2 \varepsilon$ has good stability properties for diffusion translates into better stability when combined with UP3, compared to AB3. The CFL constraints obtained for different time-stepping algorithms combined with third-order upwind scheme are reported in Tab. 2.

Among the schemes based on uncoupled space-time approaches (i.e. method of lines), the RK3 scheme is equally robust for advective and diffusive processes while other alternatives have weaknesses in either of the two cases. In Tab. 2 , the efficiency factors (i.e. $\alpha_{\mathrm{adv}}^{\star}$ divided by $n_{\text {nrhs }}$ the number of evaluation of 


\begin{tabular}{|c|c|c|c|c|c|c|c|}
\hline \hline Time-scheme for advection & $n_{\text {rhs }}$ & \multicolumn{4}{|c|}{ Stability constraints } & \multicolumn{2}{c|}{ Efficiency } \\
\hline \hline & & $\boldsymbol{\alpha}_{\mathbf{c} 2}^{\star}$ & $\boldsymbol{\alpha}_{\mathbf{u p 3}}^{\star}$ & $\boldsymbol{\alpha}_{\mathbf{C o 4}}^{\star}$ & $\boldsymbol{\beta}=\frac{\boldsymbol{\alpha}_{\mathbf{u p} 3}^{\star}}{\boldsymbol{\alpha}_{\mathbf{c} 2}^{\star}}$ & UP3-C2 & UP3-Co4 \\
\hline LFRA $(\nu=0.1)$ & 1 & 0.904 & 0.472 & 0.522 & 0.522 & 0.187 & 0.163 \\
LFAM3 & 2 & 1.587 & 0.871 & 0.916 & 0.548 & 0.171 & 0.148 \\
AB2 $(\varepsilon=0.1)$ & 1 & 0.503 & 0.554 & 0.29 & 1.108 & 0.178 & 0.141 \\
AB3 & 1 & 0.724 & 0.397 & 0.418 & 0.548 & 0.155 & 0.135 \\
RK3 & 3 & 1.73 & 1.626 & 1 & 0.93 & 0.183 & 0.150 \\
\hline & & $\alpha_{\text {LW }}^{\star}$ & $\alpha_{\mathbf{Q} \text { 33 }}^{\star}$ & $\boldsymbol{\alpha}_{\text {Co4st }}^{\star}$ & & & \\
\hline Coupled space-time & 1 & 1 & 1 & $\infty$ & 1 & 1 & 1 \\
\hline \hline
\end{tabular}

Table 2

CFL stability criteria $\alpha_{\mathrm{c} 2}^{\star}, \alpha_{\mathrm{up} 3}^{\star}$ and $\alpha_{\mathrm{Co} 4}^{\star}\left(\right.$ resp. $\alpha_{\mathrm{LW}}^{\star}, \alpha_{\mathrm{QK} 3}^{\star}$ and $\alpha_{\mathrm{C} \text { 4st }}^{\star}$ in the coupled space-time case) for different time discretizations commonly used in numerical models, $\beta$ is the ratio $\alpha_{\mathrm{up} 3}^{\star} / \alpha_{\mathrm{c} 2}^{\star}$. The efficiency for each scheme is defined here as $\frac{\alpha_{\mathrm{up} 3}^{\star}}{2+\beta}$ divided by the number of computations of the right hand side $n_{\mathrm{rhs}}$. Acronyms and parameters are defined in Tab. 1.

the right hand side) are reported for different space-time discretizations. More precisely, the efficiency factor in the three dimensional case is defined here as $\frac{\alpha_{\text {up } 3}^{\star}}{n_{\text {nrhs }}(2+\beta)}$ to account for the fact that schemes with different CFL criteria are used in the horizontal and the vertical. When considering the efficiency in a three-dimensional framework (i.e. centered in the vertical and upwind biased in the horizontal) the coupled space-time approach is significantly more efficient than any existing alternatives. This statement is even more true when using a fourth-order compact scheme in the vertical (Tab. 2). More generally, if high-order advection schemes are considered a priority, the space-time approach provides by far the best efficiency among existing schemes (with a more compact stencil, App. C.2) at the expanse of the difficulties due to the use of a directional splitting when applied to momentum equations (Sec. 2.4.1).

It is worth mentioning that large stability range is an important property but accuracy considerations are also crucial to assess the adequacy of a given numerical scheme. For example, the Semi-Lagrangian Semi-Implicit (SLSI) methods widely used in atmospheric models allow time-steps about ten times larger that of their Eulerian counterparts. However, their issues to satisfy conservation properties, their very high cost per time-step, and their strong damping of well-resolved scales make them generally less efficient than Eulerian formulations (Skamarock, 2011).

\subsubsection{Comments on the use of a Flux Corrected Transport Algorithm}

In NEMO and HYCOM, advection is often discretized using a Flux Corrected Transport (FCT) algorithm (Zalesak, 1979). The FCT procedure proved to be especially useful for the advection of passive tracers (Lévy et al., 2001) and layer thickness in isopycnic models. In this case two important points emphasized in this section are of primary importance to maintain good efficiency :

(i) the temporal scheme should have good stability properties for diffusion (because the monotone scheme in the FCT procedure is a first-order upwind scheme). For example, if a Leapfrog scheme is used the CFL stability criteria would drop from $\alpha_{\mathrm{adv}}^{\star}=0.904$ to $\alpha_{\mathrm{adv}}^{\star}=0.5$ (i.e. the stability limit of an upwind scheme integrated over $2 \Delta t$ ) when an FCT scheme is used.

(ii) in the multidimensional case, transverse terms are essential to maintain good stability. The Corner 

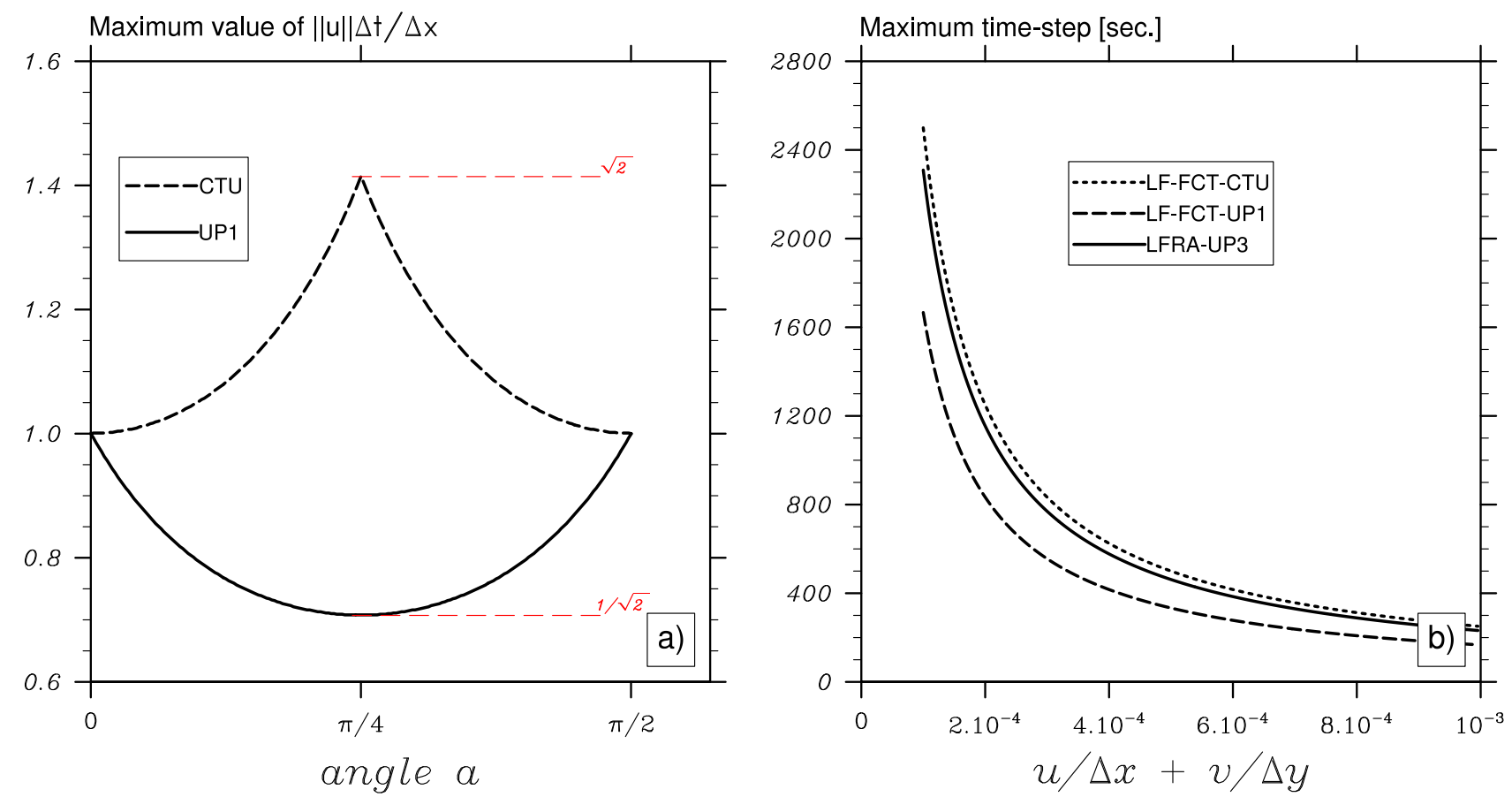

Fig. 2. a) Maximum value of $\frac{\|\mathbf{u}\| \Delta t}{\Delta x}$ (assuming $\Delta x=\Delta y$ and $\|\mathbf{u}\|=\sqrt{u^{2}+v^{2}}, u=\|\mathbf{u}\| \cos a, v=\|\mathbf{u}\| \sin a$ ) with respect to the angle $a$ between the computational grid and the flow, for the corner-transport upwind method (Colella, 1990) (dashed line) and the two-dimensional upwind scheme (solid line). $a=0$ and $a=\pi / 2$ mean that the flow is aligned with the grid whereas $a=\pi / 4$ indicates a flow oriented diagonally across the mesh. Value 1 in the vertical axis represents the maximum time-step allowed in the one-dimensional case. This value must be decreased by a factor $1 / \sqrt{2}$ in the two-dimensional case when a simple first-order upwind scheme is used. b) Maximum time-step allowed for the three dimensional integration of a Leapfrog scheme with different space discretizations with respect to $u / \Delta x+v / \Delta y$, assuming that $w / \Delta z=2(u / \Delta x+v / \Delta y)$. The acronyms are defined in Tab. 1 . In the case LFRA-UP3 a second-order advection scheme is assumed in the vertical.

Transport Upwind (CTU) method (Colella, 1990) should be preferred over an upwind scheme built on simple applications of the one-dimensional fluxes in each spatial directions. Indeed, in $2 \mathrm{D}$, the CTU scheme allows a time-step $\sqrt{2}$ times larger while preserving the monotonicity property (Fig. 2 , a). In the $3 \mathrm{D}$ case, it can be shown that the CTU allows a time-step $\sqrt{3}$ times larger compared to the simple first-order upwind scheme. If CTU is used, the maximum time-step allowed for a Leapfrog scheme would be equivalent between an LFRA-UP3 scheme and an LFRA-FCT scheme (Fig. 2, b)

Even if it is generally not necessary to consider multidimensional correction terms in the case of a method of lines, recent work by Sescu et al. $(2008,2010)$ emphasized an improvement in terms of stability and isotropy error when multidimensional cross-terms are added to the leapfrog, Runge-Kutta, or AdamsBashforth schemes in the traditional finite-difference sense. More recently, Shukla and Giri (2014) showed that removing the directional bias from the lowest-order term in the truncation error in a finite volume framework drastically reduces grid orientation dependence (a.k.a. anisotropic errors) in the computed solution.

At this point we have presented typical values of the CFL numbers $\alpha_{\mathrm{adv}}^{\star}$ and $\alpha_{\mathrm{igw}}^{\star}$ that can be derived from space-time discretizations used in existing numerical models. In the following section, we use those results to discuss the process limiting the time-step of numerical simulations with respect to the horizontal/vertical resolution. 


\section{Numerical Stability and Grid Spacing}

The aim of this section is to derive typical values for the quantities $\sqrt{c_{1}^{2}\left(\frac{1}{\Delta x^{2}}+\frac{1}{\Delta y^{2}}\right)}, u / \Delta x, v / \Delta y$ and $w / \Delta z$, with respect to the resolution. Those quantities correspond to Courant numbers divided by the time-step. To do so, we use a hierarchy of global and regional numerical solutions on which we apply offline diagnostics to compute the quantities of interest in order to analyze the stability constraints. This suite of diagnostics ${ }^{6}$ is applied to a set of numerical simulations with several horizontal/vertical resolutions and different numerical models including the global NEMO ORCA $1^{\circ}\left(\mathrm{ECMWF}^{7}\right) 1 / 2^{\circ}$ (Molines et al., 2007), $1 / 4^{\circ}$ (Dussin et al., 2012) and $1 / 12^{\circ}$ (Lecointre et al., 2011), the basin-scale ROMS Pacific configuration (Lemarié et al., 2012b), and two regional North-East Atlantic $1 / 36^{\circ}$ configurations (Charria, 2011; Cailleau et al., 2012) using the Model for Applications at Regional Scale (MARS3D, Lazure and Dumas, 2008) and NEMO. In the remainder we use those configurations in such a way that our study is not specific to NEMO, ROMS or MARS3D, but virtually applies to any numerical code.

\subsection{IGW phase speed}

First, the phase speed $c_{1}$ of the fastest internal gravity wave is considered. Details of the computation are provided in App. A. As expected, $c_{1}$ shows modest sensitivity to the horizontal/vertical resolution of the numerical models (Fig. 3) and a maximum value around $3.6 \mathrm{~m} \mathrm{~s}^{-1}$ is a good estimate. Results shown in Fig. 3 are consistent with the study of Chelton et al. (1998) based on climatological data. $c_{1}$ increases from $2.2 \mathrm{~m} \mathrm{~s}^{-1}$ near the eastern boundaries to $3.2 \mathrm{~m} \mathrm{~s}^{-1}$ near the western boundaries due to the stronger stratification in the western part of the basins. At high-latitudes the value of $c_{1}$ decreases to $1 \mathrm{~m} \mathrm{~s}^{-1}$ due to weakly stratified waters. As noted by Chelton et al. (1998), the phase speed associated with the first baroclinic vertical mode shows very small temporal variations (not shown). Results obtained for regional operational configurations including tidal currents show a sensitivity to the nature of the vertical discretization (Fig. 4). For those regional configurations significant differences in $c_{1}$ are clearly visible in both shallow and deep areas when comparing a $\sigma$ and a $z$ coordinate model. For shallow regions, faster phase speeds are observed with a $\sigma$-coordinate. We can suggest two reasons for those differences: different surface forcings leading to significantly different vertical stratifications or the interaction between slow and fast modes because slow modes are better resolved in shallow regions when using a $\sigma$-coordinate. Unlike a $\sigma$-coordinate, a $z$-coordinate model can leave the water column with very poor vertical resolution on the shelf with the ability to resolve only very few vertical modes. In the abyssal plain, differences may be due to pressure gradient errors and/or an excess of diapycnal mixing in the $\sigma$-coordinate configuration. From Fig. 3, it is accurate and robust to choose $c_{1}=3.6 \mathrm{~m} \mathrm{~s}^{-1}$ in (1.5) to evaluate the stability constraint imposed by the propagation of internal gravity waves. Maximum values of $\sqrt{c_{1}^{2}\left(\frac{1}{\Delta x^{2}}+\frac{1}{\Delta y^{2}}\right)}$ accounting for the grid stretching are given in Tab. 3 for various configurations. Using those values, it is possible to anticipate that IGW propagation becomes more restrictive than the Coriolis term for resolutions finer than

\footnotetext{
${ }^{6}$ In the framework of the COMODO project, a CF-compliant file format for NetCDF oceanic model outputs has been specified to facilitate the exchange of data and the development of interoperable diagnostics and post-processing tools called pycomodo tools http://pycomodo.forge.imag.fr/. Those tools can be applied to any model output using the COMODO file format whatever its vertical or horizontal grid. A large part of the diagnostics presented in this paper are available (or under development) in the pycomodo tools (script courant.py).

7 European Centre for Medium-Range Weather Forecasts
} 

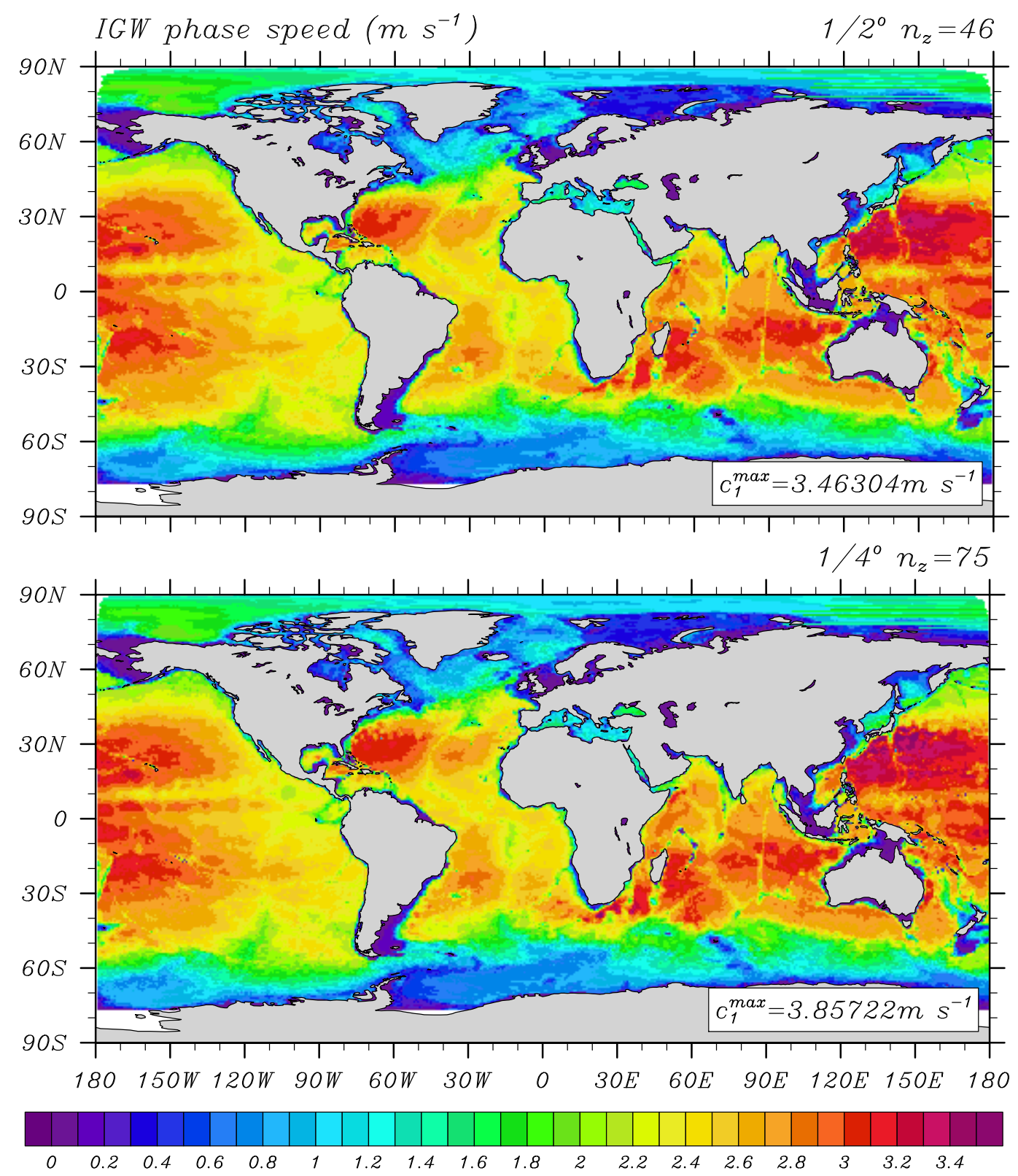

Fig. 3. Phase speed $\left[\mathrm{m} \mathrm{s}^{-1}\right]$ associated with the first baroclinic vertical mode, for a global simulation with $1 / 2^{\circ}$ resolution and 46 vertical levels (top) and with $1 / 4^{\circ}$ resolution and 75 vertical levels (bottom). Both simulations were carried out using the same numerical model, initial conditions and surface forcings.

$1 / 3^{\circ}$ (Fig. 5), and is always more restrictive than (horizontal) diffusive and viscous terms. 


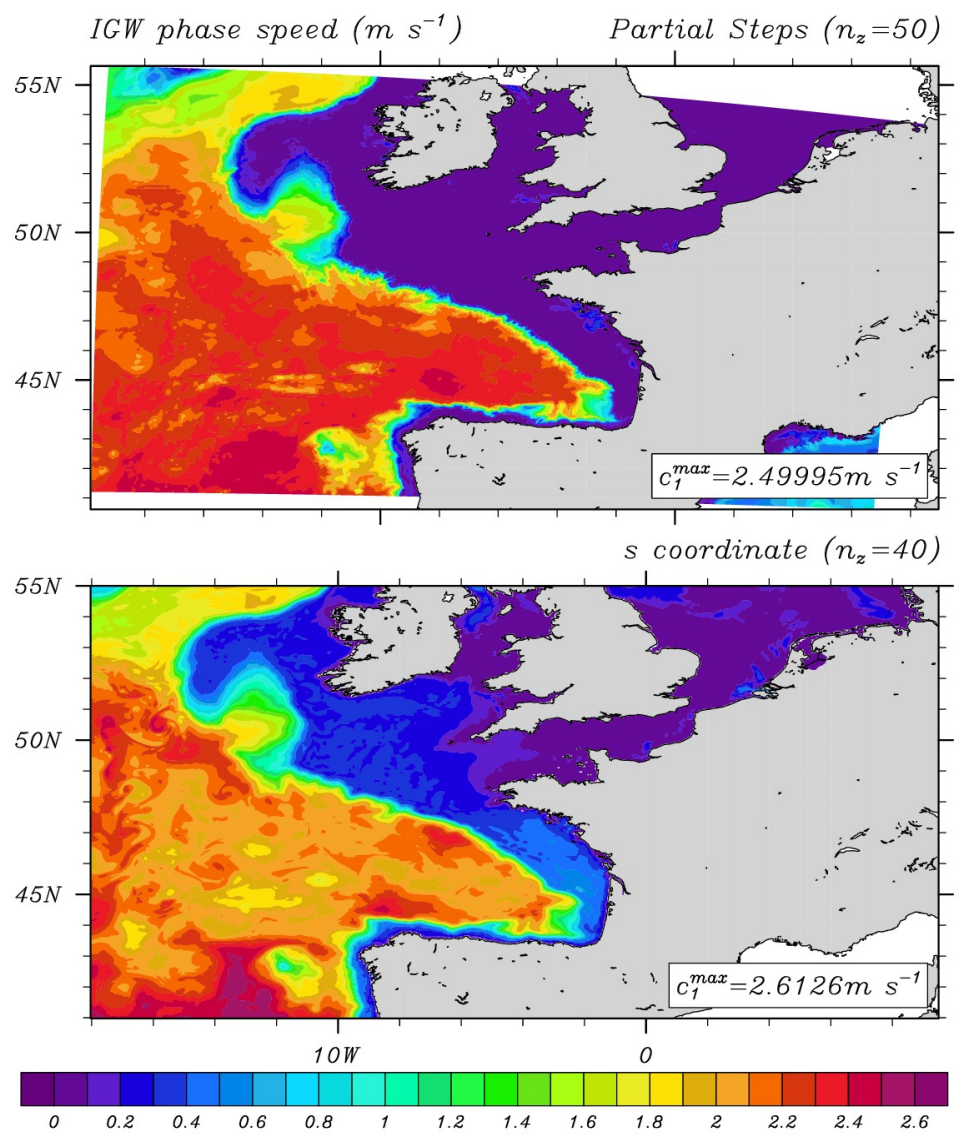

Fig. 4. Phase speed $\left[\mathrm{m} \mathrm{s}^{-1}\right]$ associated with the first baroclinic vertical mode, for regional simulations using the NEMO Partial steps model (top) and the MARS3D s-coordinate model (bottom). Note that besides the vertical discretization, those models differ in many other aspects like time-stepping, turbulent closures, etc.

\begin{tabular}{|c|c|c|c|c|c|c|c|}
\hline \hline Grid spacing & $1^{\circ}$ & $1 / 2^{\circ}$ & $1 / 2^{\circ}(\mathrm{PAC})$ & $1 / 4^{\circ}$ & $1 / 12^{\circ}$ & \multicolumn{2}{|c|}{$1 / 36^{\circ}(\mathrm{NEA})$} \\
\hline Vert coord. $\left(n_{z}\right)$ & $\mathrm{PS}(42)$ & $\mathrm{PS}(46)$ & $\sigma(50)$ & $\operatorname{PS}(75)$ & $\operatorname{PS}(46)$ & $\sigma(40)$ & PS $(50)$ \\
\hline$\alpha_{\mathrm{igw}} / \Delta t\left[\mathrm{~s}^{-1}\right]$ & $5.3 \times 10^{-5}$ & $1.2 \times 10^{-4}$ & $8.5 \times 10^{-5}$ & $2.65 \times 10^{-4}$ & $6.7 \times 10^{-4}$ & $1.4 \times 10^{-3}$ & $1.5 \times 10^{-3}$ \\
\hline$\left(\alpha_{\mathrm{adv}}^{x}+\alpha_{\mathrm{adv}}^{y}\right) / \Delta t\left[\mathrm{~s}^{-1}\right]$ & $2.98 \times 10^{-5}$ & $7.5 \times 10^{-5}$ & $4.5 \times 10^{-5}$ & $2 \times 10^{-4}$ & $6 \times 10^{-4}$ & $3.4 \times 10^{-3}$ & $1.5 \times 10^{-3}$ \\
\hline$\alpha_{\mathrm{adv}}^{z} / \Delta t\left[\mathrm{~s}^{-1}\right]$ & $7.82 \times 10^{-5}$ & $1 \times 10^{-4}$ & $1.8 \times 10^{-4}$ & $5.5 \times 10^{-4}$ & $1.2 \times 10^{-3}$ & $1.5 \times 10^{-2}$ & $4.5 \times 10^{-3}$ \\
\hline
\end{tabular}

Table 3

Maximum Courant number divided by $\Delta t$ for internal gravity waves, horizontal advection, and vertical advection in the case of several model configurations from $1 / 2^{\circ}$ to $1 / 36^{\circ}$ resolutions. The acronyms are PAC $=$ Pacific, NEA $=$ North-East Atlantic, PS=Partial-Steps. 
a)

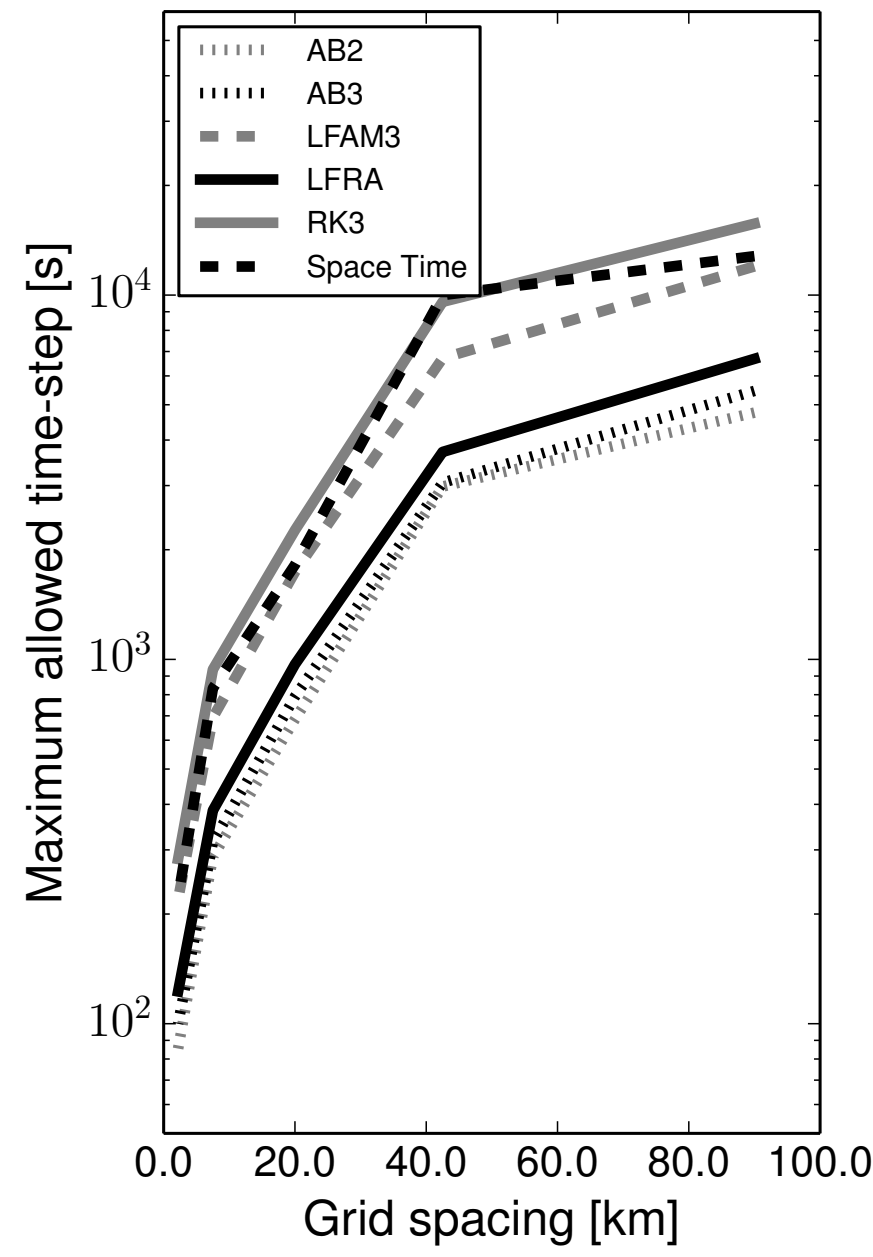

b)

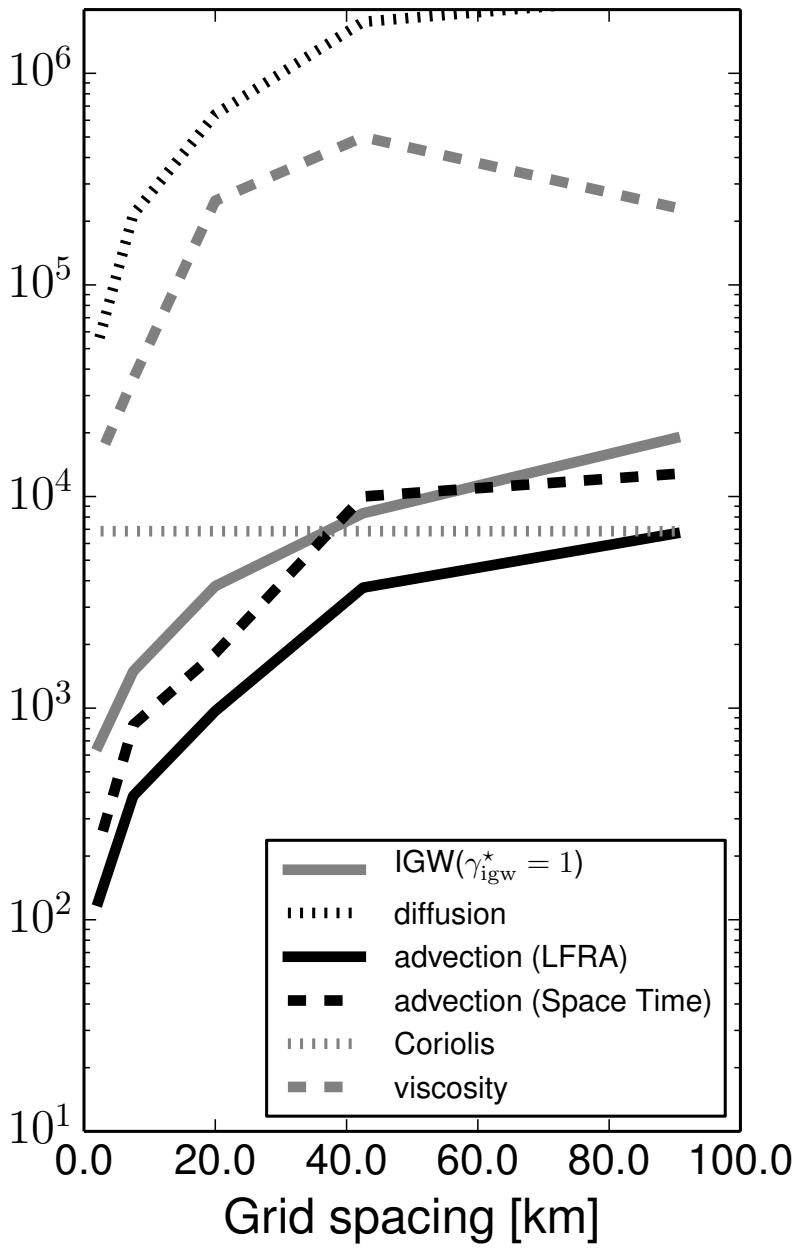

Fig. 5. a) Maximum time-step $\Delta t$ allowed for advection depending on the space-time discretizations described in Sec. 2.4. b) Maximum $\Delta t$ allowed for each process among diffusivity, viscosity, rotation, advection and internal gravity waves using a hierarchy of ORCA configurations. The grid spacing for global configurations is chosen to be representative of the resolution at mid-latitudes. The stability condition for the Coriolis term is computed as $f \Delta t \leq \alpha_{\text {cor }}$ with $\alpha_{\text {cor }}=1$. Results are obtained for a third-order upwind scheme in the horizontal and a second-order centered scheme in the vertical. The acronyms are $\mathrm{LF}=$ leapfrog, $\mathrm{RA}=$ Robert-Asselin filter, $\mathrm{AM}=\mathrm{Adams}-\mathrm{Moulton}$, $\mathrm{AB}=$ Adams-Bashforth, RK=Runge-Kutta, IGW = Internal Gravity Waves. 


\subsection{Three-dimensional Advection}

Besides internal gravity waves, we anticipate that the more restrictive process for realistic configurations is three-dimensional advection. In Tab. 3, we report the values of $\left(\alpha_{\mathrm{adv}}^{x}+\alpha_{\mathrm{adv}}^{y}\right) / \Delta t$ and separately of $\alpha_{\mathrm{adv}}^{z} / \Delta t$ for different resolutions and vertical coordinate systems. Considering that the horizontal advective CFL approximately equals $\alpha_{\mathrm{igw}}^{\star}$, we note that, for a $z$ coordinate model, internal gravity waves are more restrictive than horizontal advection only for resolutions coarser than $1 / 4^{\circ}$. However, even for lowresolution configurations at $1 / 2^{\circ}$, vertical advection is always more restrictive making three-dimensional advection the process imposing the time-step of global configurations (Fig. 5). Same conclusion holds with a $\sigma$-coordinate except that the stability constraint associated with advection is more severe because of the larger dia-surface velocities compared to a $z$-coordinate paradigm.

Fig. 6 and 7 show the process locally limiting the time-step for two global configurations at $1 / 2^{\circ}$ and $1 / 4^{\circ}$ resolution. At those resolutions most grid points are constrained by IGW propagation or rotation. However, the time-step of the model is imposed by advection which occurs at very few hot spots that can be found off Somalia and South Africa (Fig. 7). As a consequence, most of the grid points are advanced with Courant numbers much smaller than the CFL criterion, which requires the behavior of numerical schemes to be studied not only close to their CFL but predominately for small Courant numbers (see App. D). This is confirmed by Fig. 8 where the repartition of Courant numbers found in a simulation is plotted. Only very few grid points are integrated with Courant numbers larger than $\alpha_{\text {adv }}^{\star} / 10$. Looking at the relative contribution of the horizontal (Fig. 8, b) and vertical (Fig. 8, c) Courant numbers to the 3D Courant number, we can remark that the few grid points responsible for the time-step limitation are associated with large Courant numbers in the vertical. As a consequence, following Fig. 8, we can anticipate that an unconditionally stable treatment of vertical advection (as suggested in Shchepetkin (2015) in the context of regional/coastal simulations) should allow a baroclinic time-step much larger for global configurations ${ }^{8}$. Indeed, the maximum Courant number in the horizontal has values between $0.25 \alpha_{\text {adv }}^{\star}$ (for a $1 / 4^{\circ}$ resolution and 75 vertical levels) and $0.5 \alpha_{\text {adv }}^{\star}$ (for a $1 / 2^{\circ}$ resolution and 46 vertical levels). For coupled space-time schemes subject to stability constraint (2.13), the time-step is restricted by the vertical Courant number only. Fig. 8,c suggests that a stability constraint based on the maximum of the Courant number in each direction (instead of the sum) allows a time-step about $20 \%$ larger.

\footnotetext{
$\overline{8}$ Implementation of an unconditionally stable vertical advection scheme in the ROMS model allowed an increase of the time-step from $6540 \mathrm{~s}$ to $7140 \mathrm{~s}$ for a $25 \mathrm{~km}$-resolution regional domain over the Benguela, and from 160s to 470 s for a $2 \mathrm{~km}$-resolution simulation over the gulf of Oman.
} 

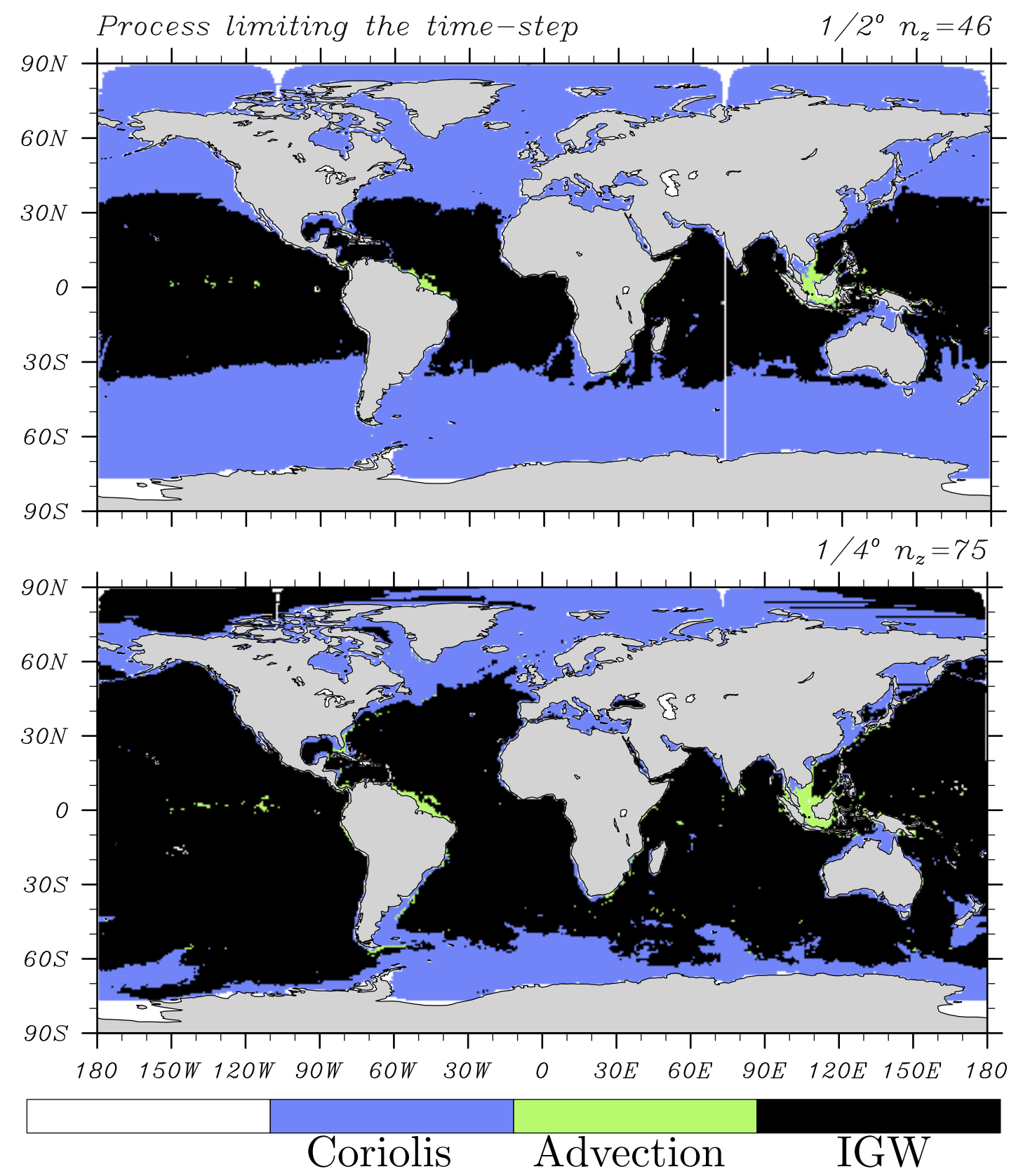

Fig. 6. Map of the process locally limiting the time-step for the $1 / 2^{\circ}$ (top) and $1 / 4^{\circ}$ (bottom) configurations among rotation (blue), three-dimensional advection (green), and internal gravity waves propagation (black). In both cases, the baroclinic time-step is set by advective processes off Somalia (at $1 / 2^{\circ}$ ) or off South Africa (at $1 / 4^{\circ}$ ). The stability conditions are computed considering $\alpha_{\mathrm{cor}}=1\left(f \Delta t \leq \alpha_{\mathrm{cor}}\right), \alpha_{\mathrm{igw}}^{\star}=1$, and $\alpha_{\mathrm{adv}}^{\star}=0.5$ (assuming stability condition (2.12)).

Using Tab. 3, it is possible to give rough estimates of the maximum time-step for a $1 / 12^{\circ}$ resolution global configuration depending on the algorithmic choices in the worst case scenario when the maximum values of $\left(\alpha_{\mathrm{adv}}^{x}+\alpha_{\mathrm{adv}}^{y}\right) / \Delta t$ and $\alpha_{\mathrm{adv}}^{z} / \Delta t$ happen at the same location. Considering a third-order upwind scheme in the horizontal combined with a second-order scheme in the vertical, we get $\Delta t \approx 390$ s for LFRA scheme and $\Delta t \approx 300 \mathrm{~s}$ for $\mathrm{AB} 2 \varepsilon$. Algorithms with third order accuracy in time generally allow larger time steps, respectively $\Delta t \approx 690$ s for LFAM3, $\Delta t \approx 945$ s for RK3, and $\Delta t \approx 830$ s for QK3 while 


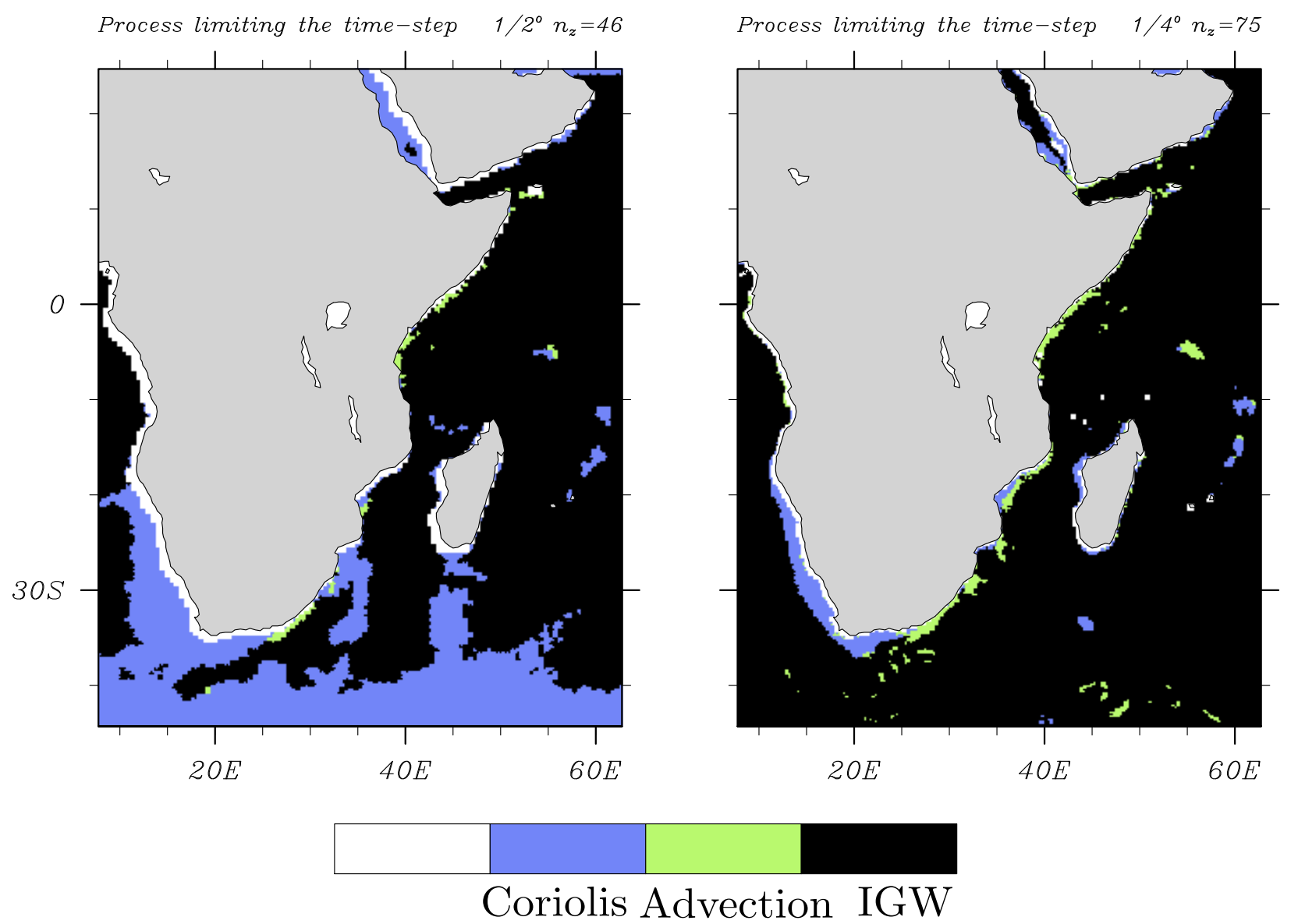

Fig. 7. Same as Fig. 6 for a zoom over east Africa. Note the few grid points off Kenya and Somalia in the left panel responsible for the time-step limitation of the $1 / 2^{\circ}$ global configuration.

$\Delta t \approx 305 \mathrm{~s}$ for $\mathrm{AB} 3$. Switching from a second-order centered scheme in the vertical to a fourth-order compact scheme has a substantial impact on the maximum allowed time step : $\Delta t \approx 280$ s for LFRA, $\Delta t \approx 190 \mathrm{~s}$ for $\mathrm{AB} 2, \Delta t \approx 230 \mathrm{~s}$ for AB3, $\Delta t \approx 500 \mathrm{~s}$ for LFAM3, and $\Delta t \approx 625 \mathrm{~s}$ for RK3. Provided that a space-time version of a fourth-order compact scheme is built (App. B), the time-step is unchanged for the one-step space-time approach (i.e. $\Delta t \approx 830 \mathrm{~s}$ ). Because those estimates of the time-step are derived considering the worst case scenario when the maximum contraint for horizontal and vertical advection happen at the same location, they systematically underestimate the time-step value that can be used in practice. For example, for the LFRA scheme our estimate is $\Delta t \approx 390$ s while the ORCA12 simulations with NEMO (i.e. the LFRA scheme) uses a time-step $\Delta t=480 \mathrm{~s}$.

Note that even if the LFAM3 scheme requires two evaluations of the right hand side, the cost per timestep is not twice the one of a Leapfrog or an Adams-Bashforth scheme that require only one evaluation of the rhs. Indeed, costly terms like vertical and lateral diffusion and viscosity, as well as the barotropic mode are computed only once per time step, outside of the LFAM3 loop. This remark virtually applies to the RK3 scheme too except that the barotropic mode is not easy to handle efficiently in the RK framework. Moreover, the computational cost of a given scheme is dependent not only on its stability condition but also on its actual implementation in the numerical code. Different implementations can lead to substantial differences in terms of memory usage (i.e. access pattern and frequency) hence computational cost. Recent results reported in Soufflet et al. (2015) seem to suggest that two numerical codes with different coding 
structures and memory access patterns can have drastically different computational cost.

At this point, we have focused our attention on terms integrated using explicit time-stepping algorithms subject to conditional stability. Because the time-step value has also great impact on the accuracy of implicit methods, it is legitimate to assess accuracy problems which may occur in numerical simulations.
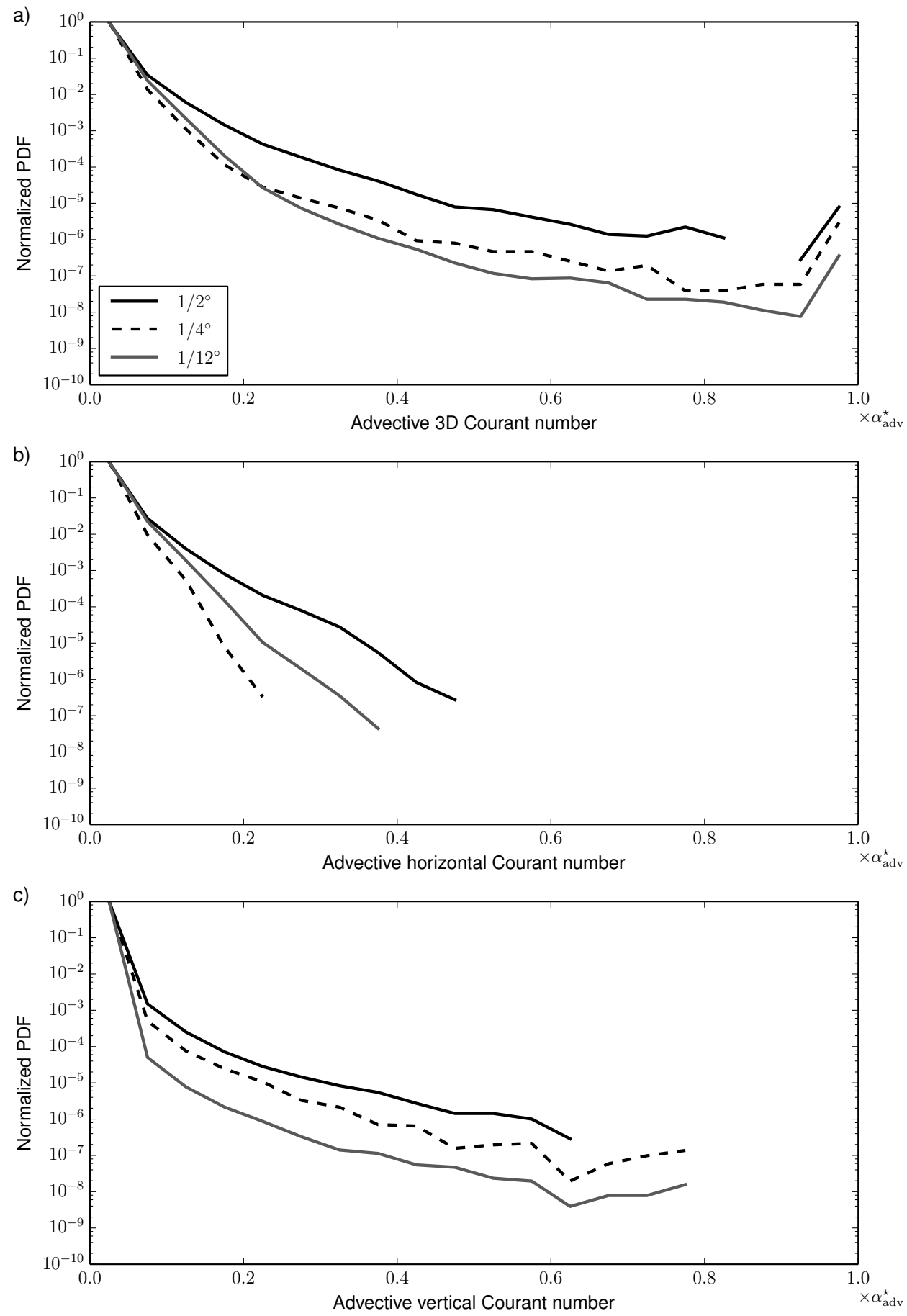

Fig. 8. Normalized Probability Density Function (PDF) of the 3D Courant number (a), horizontal Courant number (b) and vertical Courant number (c) for the $1 / 2^{\circ}$ (black lines), $1 / 4^{\circ}$ (dashed lines), and $1 / 12^{\circ}$ (gray lines) global configurations. Note the log-scale in the vertical axis and the multiplication of the horizontal axis by the CFL criterion $\alpha_{\mathrm{adv}}^{\star}$. 


\subsection{Turbulent vertical diffusion}

Due to the small vertical, relative to horizontal, grid distance typically used in numerical models vertical terms can impose a severe restriction on the time step when explicit-in-time methods are used. To circumvent this problem, most of the vertical terms (with the exception of vertical advection) are systematically treated implicitly in time using a backward Euler scheme. In this case, stability is not an issue but accuracy problems may occur in stiff situations (i.e. the actual Courant number is much larger than the explicit CFL). The exact damping rate for the diffusion equation with diffusivity $\kappa$ is $\omega^{e}=\kappa k_{z}^{2}=\sigma^{(2)} \theta^{2} / \Delta t$ (e.g. Cushman-Roisin and Beckers, 2011, Chap 5), with $\sigma^{(2)}=\kappa \Delta t / \Delta z^{2}$ the vertical parabolic Courant number and $\theta=k_{z} \Delta z$ the normalized wavenumber. The numerical damping rate for a numerical scheme with amplification factor ${ }^{9} \mathcal{A}$ is $\omega^{\text {num }}=-\log \left|\mathcal{A}\left(\sigma^{(2)}, \theta\right)\right| / \Delta t$. Using $\omega^{e}$ and $\omega^{\text {num }}$, we can suggest an effective diffusivity $\kappa^{\mathrm{eff}}$ which would account for numerical errors. Let us define $\mathcal{E}=-\log |\mathcal{A}| /\left(\sigma^{(2)} \theta^{2}\right)$ the ratio between the numerical and the exact damping rate. The effective diffusivity is the value of diffusivity in the exact damping $\omega^{e}=\kappa^{\text {eff }} k_{z}^{2}$ such that $\omega^{\text {num }} / \omega^{e}=1$ (i.e. $\left(\theta^{2} \Delta t / \Delta z^{2}\right) \kappa^{\text {eff }}=-\log |\mathcal{A}|$ ). Replacing $-\log |\mathcal{A}|$ by $\left(\frac{\theta^{2} \Delta t}{\Delta z^{2}}\right) \kappa^{\text {eff }}$ in the expression for $\mathcal{E}$ we get

$$
\mathcal{E}=-\frac{\log |\mathcal{A}|}{\sigma^{(2)} \theta^{2}}=\frac{\left(\frac{\theta^{2} \Delta t}{\Delta z^{2}}\right) \kappa^{\mathrm{eff}}}{\sigma^{(2)} \theta^{2}}=\frac{\kappa^{\mathrm{eff}}}{\kappa} .
$$

The value of $\kappa^{\text {eff }}$ is the diffusivity in the continuous equation which would give the same damping as the numerical damping. For the Euler backward scheme, using (3.1) we see that for large vertical parabolic Courant numbers (i.e. larger than $1 / 2$ the stability constraint of the Euler forward scheme) the ratio $\kappa / \kappa^{\text {eff }}$ is significantly larger than 1 even for well-resolved scales (Fig. 9). This indicates that the numerical scheme dampens less fast than the physical process because $\kappa \gg \kappa^{\text {eff }}$ for $\sigma^{(2)} \gg 1 / 2$. From Fig. 9 we can anticipate that realistic numerical simulations could suffer from this underestimation of the damping as typical values for the vertical parabolic number in the boundary layer are generally around $\sigma^{(2)} \approx 10$ (e.g. for $\kappa=10^{-2} \mathrm{~m}^{2} \mathrm{~s}^{-1}, \Delta t=1000 \mathrm{~s}$, and $\Delta z=1 \mathrm{~m}$ ). The issue is further compounded by the fact that global ORCA simulations generally use convective adjustment in the surface boundary layer via an enhanced vertical diffusion (EVD) with $\kappa^{\mathrm{evd}}=10 \mathrm{~m}^{2} \mathrm{~s}^{-1}$. In Fig. 10, the value of $\kappa / \kappa^{\mathrm{eff}}=1 / \mathcal{E}\left(\sigma^{(2)}, \theta\right)$ obtained for realistic global simulations considering an implicit Euler scheme is plotted using $\sigma^{(2)}=\bar{\sigma}^{(\mathrm{mld})}$ the vertically averaged value in the mixed layer ${ }^{10}$ and $\theta=2 \pi / N_{\text {mld }}$ with $N_{\text {mld }}$ the number of grid points in the mixed layer. Results are shown for the average over the convective and non-convective seasons based on 5-day average outputs. As expected, values of $\kappa / \kappa^{\text {eff }}$ are systematically larger than 1 . The effective diffusivity is smaller than the turbulent diffusivity by one or two orders of magnitude. Because of the implicit treatment of vertical diffusion, the amount of diffusion in the vertical is significantly less that what we would expect from physical principles (i.e. from the turbulent closure and/or convective adjustment schemes). In the convective case, the effective diffusivity is generally two orders of magnitude smaller than $\kappa^{\text {evd }}$. We believe it is not worrisome because the water column seems to be very efficiently mixed anyway. Indeed, recent experiments using a non-penetrative ${ }^{11}$ convection scheme (Madec et al., 1991) showed very similar results compared to the EVD scheme. However, during the non-convective seasons, values of $\kappa / \kappa^{\text {eff }}$ between 2 and 10 are not unusual (not shown). Such values are significant and should

${ }_{9}^{9}$ For the backward Euler scheme, the amplification factor is $\mathcal{A}=\left\{1+2 \sigma^{(2)}(1-\cos \theta)\right\}^{-1}$.

${ }^{10}$ The mixed layer depth is defined as the depth where density equals the $10 \mathrm{~m}$ depth density plus $0.03 \mathrm{~kg} \mathrm{~m}^{-3}$.

${ }^{11}$ This algorithm is applied at each time step and mixes downward instantaneously the statically unstable portion of the water column using an iterative procedure until the density structure becomes neutrally stable. 


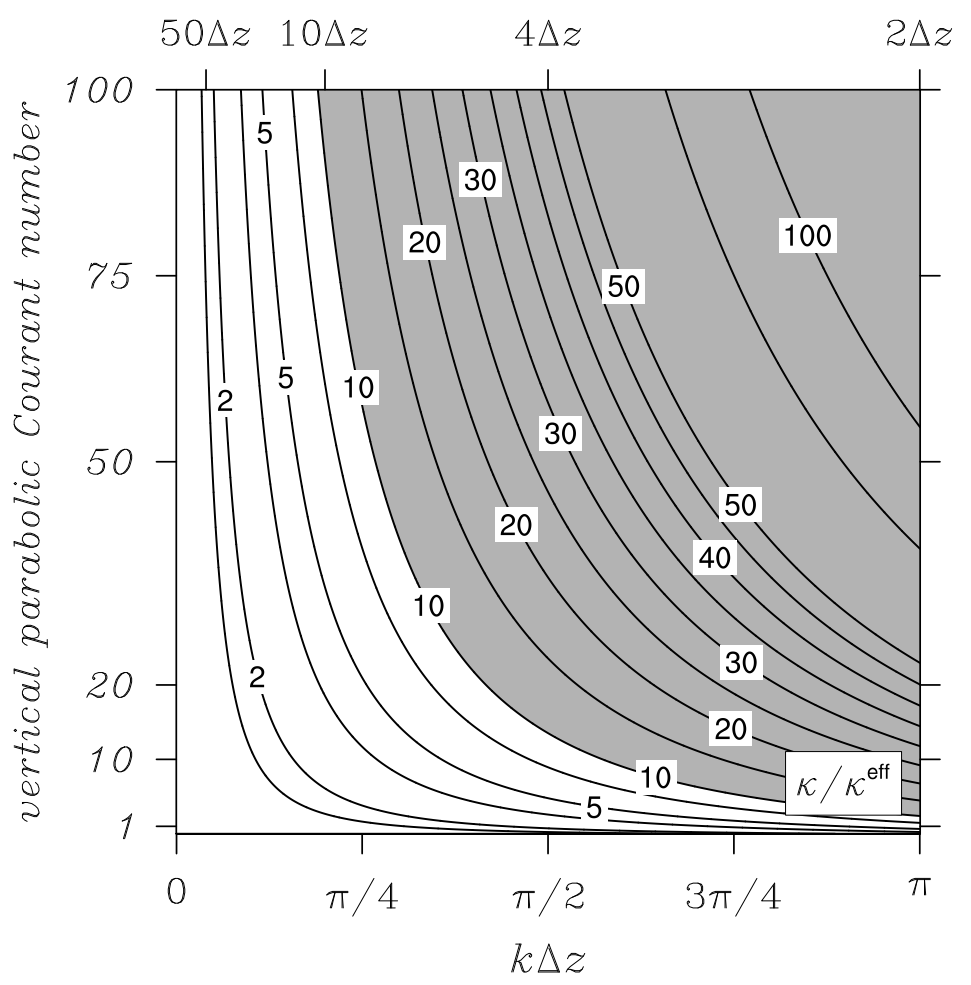

Fig. 9. Ratio between the turbulent vertical diffusivity $\kappa$ and the effective diffusivity $\kappa^{\text {eff }}$ with respect to the vertical parabolic Courant number $\sigma^{(2)}$ and the normalized wavenumber $k_{z} \Delta z$ as defined in (3.1). Time discretization is a backward Euler scheme. Values of $\kappa / \kappa^{\text {eff }}$ larger than 1 means that, because of numerical errors, the damping seen by the model is smaller than the theoretical damping associated to the diffusivity value $\kappa$.

motivate further investigation of this issue. Going from 46 to 75 vertical levels worsens the problem. It would be instructive to integrate vertical diffusion using a time-splitting approach (i.e. few small timesteps to advance diffusion within a larger baroclinic time step) to get a better sense of the impact on the physical solution. This point should be examined further in future work.

Note that isoneutral diffusion is also influenced by those numerical errors for large vertical parabolic Courant numbers. Indeed, the vertical component of the isoneutral diffusion tensor is integrated using a backward Euler scheme while other components are integrated explicitly. Large grid slope ratios (i.e. the isoneutral slope scaled by $\Delta x / \Delta z$ ) translate into large values for the vertical term of the diffusion tensor, thus very large vertical parabolic Courant numbers which lead to very inaccurate numerical integration for this component of the tensor. The ultimate consequence is that for large grid slope ratios there will be large numerical errors (due to the time-stepping) for the vertical term of the tensor leading to inaccuracies in the orientation of diffusion.

\section{Summary remarks}

Oceanic global circulation models, originally developed for low-resolution global configurations, are now being routinely used for high-resolution global or regional multi-scale simulations. As a consequence, numerical methods and physical parameterizations have evolved to accommodate to this new range of applications. However, the work on time-stepping algorithms has been quite sporadic, very few profound change took place. A first step in this direction is to define the key guidelines for the development of temporal schemes in future multi-purpose oceanic models. With this objective in mind, the present study 

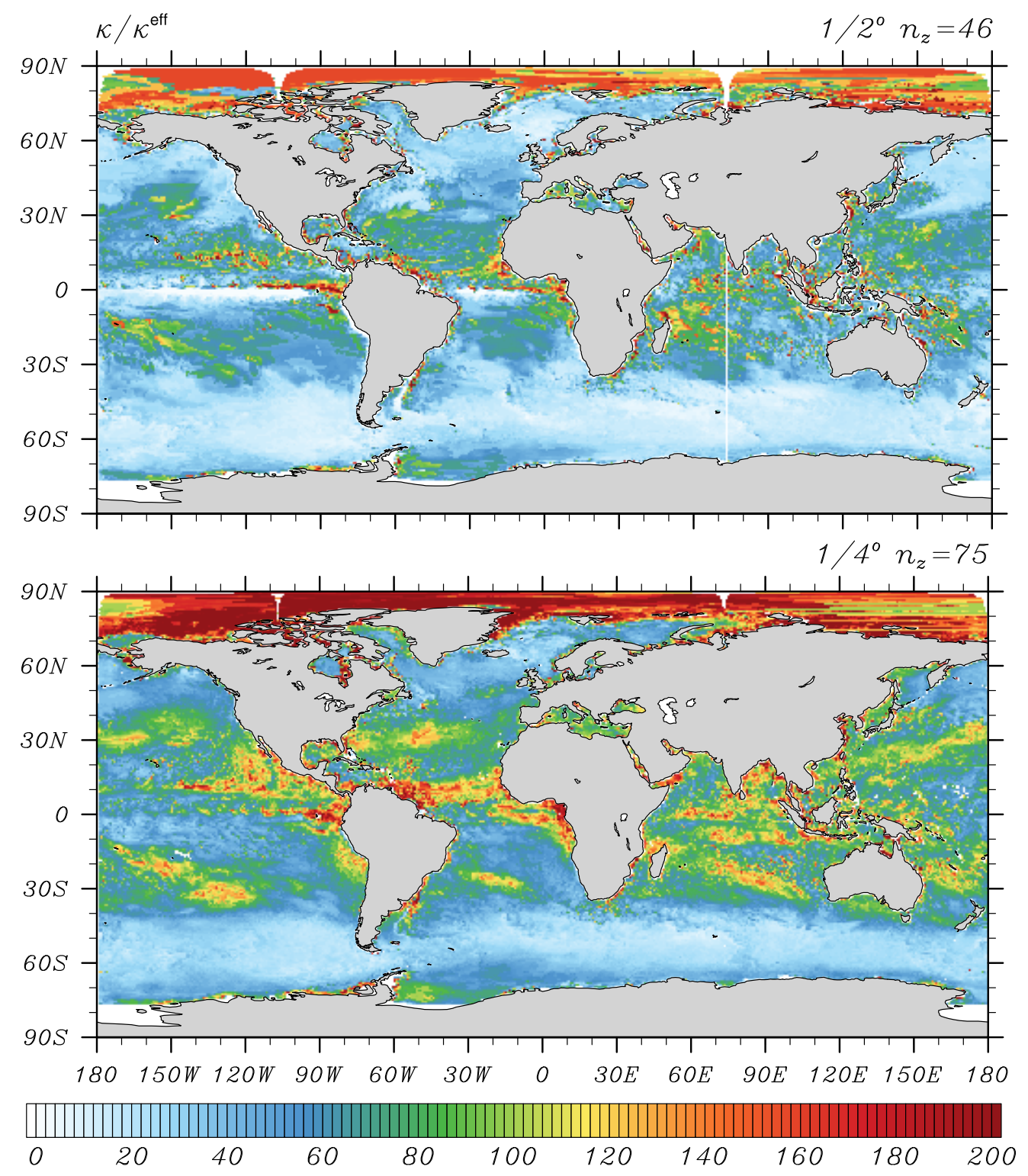

Fig. 10. Ratio between the turbulent vertical diffusivity $\kappa$ and the effective diffusivity $\kappa^{\text {eff }}$ for each water column of the $1 / 2^{\circ}$ (top) and the $1 / 4^{\circ}$ (bottom) configurations. $\kappa^{\text {eff }}$ is the diffusivity in the continuous equation which would give the same damping as the numerical damping (ideally we should get $\kappa / \kappa^{\text {eff }}=1$ ). Areas shaded in yellow and red indicate regions with large numerical errors in the computation of vertical diffusion. The value of $\kappa / \kappa^{\text {eff }}$ is computed using (3.1) with $\sigma^{(2)}=\bar{\sigma}^{(\mathrm{mld})}$ the averaged value of the vertical parabolic Courant number in the mixed layer and $\theta=2 \pi / N_{\text {mld }}$ with $N_{\text {mld }}$ the number of grid points in the mixed layer.

aims at improving our understanding of the requirements from numerical methods to better represent the mesoscale in global models. Our findings are summarized below.

\subsection{Stability constraints for oceanic numerical models}

First, the different constraints applying on the time-step of oceanic models have been investigated. We have developed a suite of offline diagnostics to predict stability and accuracy limits. Those diagnostics 
have been applied to a set of global and regional simulations with different horizontal/vertical resolutions. As noted previously in Griffies and Adcroft (2008), the maximum time-step allowed for the integration of three-dimensional advection greatly depends on the details of the space-time discretizations (Fig. 5,

a). The values of the CFL numbers $\alpha_{\mathrm{adv}}^{\star}$ for advection and $\alpha_{\mathrm{igw}}^{\star}$ for internal gravity waves, and of the ratio $\beta$ between the CFL criteria in the horizontal and vertical directions must be known to anticipate the process imposing the stability limit. An accurate estimate of $\alpha_{\mathrm{adv}}^{\star}, \alpha_{\mathrm{igw}}^{\star}$ and $\beta$ requires a careful study in a space-time framework unlike most of the existing studies carried out in a semi-discretized framework (i.e. with "perfect" numerical schemes in space). Indeed, we showed that, when high-order spatial schemes are used, CFL criteria arising from a space-time study can differ significantly from the CFL criteria found in the semi-discretized case.

Considering the typical CFL criteria associated with the space-time discretizations currently used in oceanic models, we found that three-dimensional advection is the process limiting the time-step of global configurations, even for resolutions as coarse as $1 / 2^{\circ}$. This limitation holds for very few isolated grid points mainly located near the coastline of Africa (Somalia and South Africa) and/or at the equator (Fig. $6,7)$. More precisely, vertical advection is the term imposing the time-step of global configurations with an Eulerian vertical coordinate at eddy-permitting and eddying resolutions. Our results suggest that an unconditionally stable treatment of vertical advection would allow a baroclinic time-step much larger for global configurations with grid spacing in the range $1 / 2^{\circ}-1 / 12^{\circ}$ (Fig. 8). In this case, propagation of internal gravity waves could be the limiting process for the time-step. This conclusion is mainly valid for global configurations. At midlatitudes, the propagation of internal gravity waves can locally limit the time-step. As a result, the time-step of a regional domain at high-latitudes would be limited by the internal gravity waves even at eddying resolution. Those remarks motivate the ongoing work on trying to extend the stability range of time-stepping algorithms for the propagation of internal gravity waves (e.g. Shchepetkin and McWilliams, 2008, Sec. 2.1).

Even if implicit time integration have no stability conditions to meet, large time-steps are not acceptable and can lead to very inaccurate representation of the underlying physical process. Large time-steps (large relative to the explicit CFL) would lead to too weak of a damping for vertical diffusion. This means that, because of numerical errors, the level of mixing seen by the model can be very different from the mixing value given by the physical parameterization (sometimes one or two order of magnitude less). This problem is expected to be more acute when increasing the vertical resolution. It would be instructive to integrate those terms using a time-splitting approach (i.e. few small time-steps to advance diffusion within a larger baroclinic time step) to get a better sense of the impact on the physical solution. This point should be examined further in future work.

\subsection{Implications for the formulation of time and space discretizations}

From our study, we can identify a few guidelines in the design of space-time discretizations in the context of oceanic numerical models :

(1) It is essential to study numerical schemes close to their functioning conditions (i.e. in a space-time approach). Time-stepping algorithms can not be designed independently from space discretizations. It can be done relatively simply using the stability diagrams presented in Fig. 1 and Sec. 2.4.2. A spacetime study would also allow a better control of the total truncation error which is important nowadays to assess more accurately the relative role of discretization errors vs physical parameterizations.

(2) Because the time-step is imposed by a few isolated hot spots (Fig. 8), the numerical schemes must be 
extremely robust to changes in Courant number. The behavior of numerical schemes should, thus, be studied not only close to their CFL but predominately for small Courant numbers.

(3) The viability of (semi)-implicit schemes for vertical advection while maintaining 3D properties (e.g. positive-definiteness) is an open question. A very promising way to handle vertical advection and significantly extend the range of stability of numerical models discretized using an eulerian vertical coordinate, without impairing accuracy, has been developed in Shchepetkin (2015). The idea is to split vertical velocity into two parts : one is treated explicitly in time using a fourth-order compact discretization in space, the other is treated using an implicit (backward) Euler scheme with firstorder upwind scheme in space. The splitting between the explicit and the implicit pieces is done based on the value of the local Courant number : if the Courant number is below the CFL criterion, vertical advection is treated explicitly with 4th-order accuracy in space. Once the Courant number exceeds the CFL criteria the excess of vertical velocity is treated implicitly with 1st-order accuracy in space-time. An interesting alternative could be the use of a vertical coordinate which separate high frequency motions (associated with large vertical velocities) handled in a lagrangian way and low frequency motions handled in an eulerian way (Leclair and Madec, 2011).

(4) Space-time discretizations must be robust and stable for advection and diffusion. Indeed, centered advection schemes are generally used in the vertical to avoid excessive diapycnal mixing and the use of dissipative advection schemes is necessary in the horizontal to control dispersive errors. We showed that the study of a simple oscillation equation (2.15) with complex frequency $\omega$ allows a detailed investigation of the properties of a given scheme (see Sec. 2.4.2).

(5) A proper representation of the energy spectrum would require the schemes to satisfy a monotonic damping property (i.e. smaller scales are always more efficiently damped than larger scales). This property is satisfied by the exact diffusion operator and we expect time-stepping algorithms combined with odd-ordered advection schemes to retain this property (see App. D).

(6) Time-stepping algorithms should naturally combine with positive-definite (resp. monotonic) transport scheme while maintaining good accuracy and stability. Note that Flux Corrected Transport (FCT) and Total Variation Diminishing (TVD) methods tend to erroneously damp smooth extrema (i.e. very well resolved scales). The motonicity-preserving method of Suresh and Huynh (1997) (adapted to the coupled space-time context by Daru and Tenaud (2004)) seems promising to avoid the loss of accuracy near extrema.

(7) An accurate propagation of internal gravity waves should be considered a priority as resolution keeps increasing. This involves not only the time-stepping algorithm but also better accuracy in the discretization of horizontal pressure gradient and continuity equation.

In Sec. 2 and App. D, a thorough study of the schemes currently used in oceanic models suggests that no time-integration scheme meets all the aforementioned requirements, for the reasons explained in next subsection.

\subsection{Critical comments on current time and space integration schemes}

The merits and flaws of existing space-time integrations are discussed in Sec. 2 and App. D. The Leapfrog (LF) scheme with Robert-Asselin (RA) filtering has been used for years in the context of global climate models especially because it has good discrete conservation properties. It is well-known that the RA filter increases phase errors, dissipates the physical mode and affects stability. Moreover, the LF-RA time-stepping algorithm can be combined only with centered advection schemes because it is unconditionally unstable for diffusive process. As a consequence, monotonicity or positive-definiteness (e.g. via a 
Flux Corrected Transport procedure) comes at a substantial cost because it must be done on $2 \Delta t$ thus dividing the CFL criteria by 2 . Moreover, the lack of damping of the computational mode for well-resolved scales has been a long-standing problem. An other choice popular for global models is the second-order time-biased Adams-Bashforth $(\mathrm{AB} 2 \varepsilon)$ scheme. However, this scheme has a very limited range of stability for centered advection schemes and turns out to be first-order accurate because of a time-shift in the extrapolation rule. In general, the AB2 computational mode does not interfere with well-resolved scales but can affect small scales.

An LF predictor step combined with an third-order Adams-Moulton (AM3) interpolation for the corrector step provides a scheme with several good properties : third-order accuracy, robustness to changes in Courant number, monotonic damping (for scales larger than $4 \Delta x$ ), good stability for centered advection schemes. However the LFAM3 scheme has two major drawbacks : there is a significant loss of stability when using odd-ordered advection schemes and the AM3 interpolation has negative weights, therefore this scheme does not maintain positive-definiteness even if the space discretization does. Moreover this scheme has a computational mode which is not efficiently damped for smaller scales close to the CFL limit.

A popular choice in numerical weather forecasting models is the third-order Runge-Kutta (RK3) scheme. This scheme is free of computational mode and has good efficiency factor because it offers good stability properties for advection and diffusion, namely for centered and upwind advection schemes. However, the RK3 has an unpredictable behavior in a large part of its stability range (approximately for Courant numbers larger than 0.9, App. D) : it does not monotonically damp scales, the leading order term of the truncation error is a biharmonic operator with positive coefficient and phase errors can be relatively large, as a consequence the scheme is not robust to changes in Courant number. However, this scheme behaves well for Courant numbers smaller than 0.9. In this range of Courant numbers, it satisfies the requirements given in previous subsection.

One-step space-time schemes are seldom used in the context of oceanic models. In a three-dimensional case, those schemes offer an efficiency factor that is unrivaled (5 times larger than that of RK3 with a more compact stencil) for dissipative and centered advection schemes. This class of scheme is free of computational mode, satisfy the monotonic damping property, and combine nicely with monotonicity constraints (e.g. Daru and Tenaud, 2004). The main drawbacks are that those schemes behave differently with respect to the Courant number (Fig. D.3, left panel) and require a directional splitting which makes their use delicate in the momentum equation. The space-time approach provides exact solutions at its CFL stability condition $\alpha_{\mathrm{adv}}=1$. Decreasing the time-step leads to rapid changes in the error mainly for $4 \Delta x$ and $2 \Delta x$ waves (Fig. D.1, right panel). However, larger scales are still well preserved.

Among the various space-time discretizations discussed in this paper, the one-step space-time approach offers an interesting compromise among the requirements we defined above. Moreover, this approach is flexible enough to easily adapt the space-time order of accuracy depending on the target application. It remains to be seen how those schemes would combine with mode-splitting and if the space-time approach can be successfully extended to the internal gravity waves propagation discretized on a C-grid (e.g. Demange et al., 2014a). 


\section{Acknowledgements}

F. Lemarié acknowledges the support of the French LEFE (Les Enveloppes Fluides et l'Environnement) MANU (méthodes MAthématiques et NUmériques) and GMMC (Groupe Mission Mercator Coriolis) programs through project CHRONOS (numerical study of time-integration schemes for oceanic models). L. Debreu, M. Honnorat and G. Madec were funded by the ANR through contract ANR-11-MONU-005 (COMODO). Authors are thankful to M. Balmaseda, A. Vidard, F. Dumas, S. Masson and J. Chanut for kindly providing their model outputs, and V. Daru and J. Le Sommer for fruitful discussions. Authors would like to express their sincere thankfulness to S. Griffies and three anonymous reviewers whose valuable thoughts and suggestions during the review process helped to clarify the manuscript.

\section{References}

Adcroft, A., Campin, J.M., Dutkiewicz, S., Evangelinos, C., Ferreira, D., Forget, G., Fox-Kemper, B., Heimbach, P., Hill, C., Hill, E., Hill, H., Jahn, O., Losch, M., Marshall, J., Maze, G., Menemenlis, D., Molod, A., 2014. MITGCM USER MANUAL, in: MIT Department of EAPS 77 Massachusetts Ave. Cambridge, MA 02139-4307. March 10, 2014. 470 pp. http://mitgcm.org/public/r2_ manual/latest/online_documents/manual.pdf.

Arbic, B.K., Richman, J.G., Shriver, J.F., Timko, P.G., Metzger, E.J., Wallcraft, A.J., 2012. Global modeling of internal tides within an eddying ocean general circulation model. Oceanogr. 25(2), 20-29.

Baldauf, M., 2008. Stability analysis for linear discretisations of the advection equation with runge-kutta time integration. J. Comp. Phys. 227(13), 6638 - 6659.

Beckers, J.M., Deleersnijder, E., 1993. Stability of a FBTCS scheme applied to the propagation of shallowwater inertia-gravity waves on various space grids. J. Comp. Phys. 108(1), 95 - 104.

Blayo, E., Debreu, L., 2005. Revisiting open boundary conditions from the point of view of characteristic variables. Ocean Modell. 9(3), $231-252$.

Bleck, R., Halliwell, G., Wallcraft, A., Carroll, S., Kelly, K., Rushing, K., 2002. HYCOM USER'S MANUAL, March 4, 2002. 211 pp. http: / / hycom.org/attachments/063_hycom_users_ manual.pdf.

Bott, A., 2010. Improving the time-splitting errors of one-dimensional advection schemes in multidimensional applications. Atmospheric Research 97, 619-631.

Brown, J.A., Campana, K.A., 1978. An economical time-differencing system for numerical weather prediction. Mon. Weather Rev. 106, 1125-1136.

Cailleau, S., Chanut, J., Lellouche, J.M., Levier, B., Maraldi, C., Reffray, G., Sotillo, M.G., 2012. Towards a regional ocean forecasting system for the IBI (Iberia-Biscay-Ireland area): developments and improvements within the ECOOP project framework. Ocean Science 8(2), 143-159.

Charria, G., 2011. Modelling the Channel and the Bay of Biscay using the MARS model. IFREMER/PREVIMER, EPIGRAM annual meeting Available online at http: //wwz.ifremer.fr/epigram/content/download/44280/626549/file/charria_ modele-obs-ifremer.pdf.

Chelton, D., deSzoeke, R., Schlax, M., Naggar, K.E., Siwertz, N., 1998. Geographical variability of the first baroclinic rossby radius of deformation. J. Phys. Oceanogr. 28, 433-460.

Colella, P., 1990. Multidimensional upwind methods for hyperbolic conservation laws. J. Comp. Phys. 87(1), $171-200$. 
Cushman-Roisin, B., Beckers, J.M., 2011. Introduction to Geophysical Fluid Dynamics: Physical and Numerical Aspects. Academic Press.

Daru, V., Tenaud, C., 2004. High order one-step monotonicity-preserving schemes for unsteady compressible flow calculations. J. Comp. Phys. 193, 563-594.

Debreu, L., Blayo, E., 2008. Two-way embedding algorithms: a review. Ocean Dyn. 58(5-6), 415-428.

Debreu, L., Marchesiello, P., Penven, P., Cambon, G., 2012. Two-way nesting in split-explicit ocean models: algorithms, implementation and validation. Ocean Modell. 49-50, 1-21.

Demange, J., Debreu, L., Marchesiello, P., Lemarié, F., Blayo, E., 2014a. Numerical representation of internal waves propagation. Research report RR-8590. INRIA. Available online at http://hal . inria.fr/hal-01063417/PDF/RR-8590.pdf.

Demange, J., Debreu, L., Marchesiello, P., Lemarié, F., Blayo, E., 2014b. On the use of a depthdependent barotropic mode in ocean models: impact on the stability of the coupled barotropic/baroclinic system. Research report RR-8589. INRIA. Available online at http://hal.inria.fr/ hal-01063414/PDF/RR-8589.pdf.

Duhaut, T., Honnorat, M., Debreu, L., 2008. Développements numériques pour le modèle MARS. Technical Report. PREVIMER report - Ref : 06/2 210 290. Available online at http://wwz.ifremer.fr/mars3d/content/download/49831/708186/file/ 2008_05_30_INRIA_debreu_final.pdf.

Durran, D.R., 2010. Numerical Methods for Fluid Dynamics With Applications to Geophysics. Springer. 2nd edition.

Dussin, R., Molines, J.M., Barnier, B., 2012. Definition of the interannual experiment ORCA025.L75GRD100, 1958-2010. Technical Report. LEGIDRA-12-04-2012. Available online at http://www . drakkar-ocean.eu/publications/reports/orca025-grd100-report-dussin.

Eden, C., Czeschel, L., Olbers, D., 2014. Toward Energetically Consistent Ocean Models. J. Phys. Oceanogr. 44, 3160-3184.

Griffies, S., Adcroft, A., 2008. Formulating the Equations of ocean models, in: Hecht, M., Hasumi, H. (Eds.), Ocean Modeling in an Eddying Regime. American Geophysical Union, USA. volume 177 of Geophysical Monograph Series, p. 350.

Griffies, S.M., 2010. Elements of MOM4p1, in: GFDL Ocean Group Technical Report No. 6. NOAA/GFDL, February 19, 2010. 444 pp.

Griffies, S.M., 2013. Ocean model numerics and physics: challenges for mesoscale eddying global climate simulations. Drakkar meeting, Grenoble, France, January 28-30 .

Griffies, S.M., Boning, C., Bryan, F.O., Chassignet, E.P., Gerdes, R., Hasumi, H., Hirst, A., Treguier, A.M., Webb, D., 2000. Developments in ocean climate modelling. Ocean Modell. 2, 123-192.

Griffies, S.M., Gnanadesikan, A., Pacanowski, R.C., Larichev, V., Dukowicz, J.K., Smith, R.D., 1998. Isoneutral diffusion in a z-coordinate ocean model. J. Phys. Oceanogr. 28(5), 805-830.

Griffies, S.M., Treguier, A.M., 2013. Chapter 20 - ocean circulation models and modeling, in: Siedler, G., Griffies, S.M., Gould, J., Church, J.A. (Eds.), Ocean Circulation and Climate A 21st Century Perspective. Academic Press. volume 103 of International Geophysics, pp. 521 - 551.

Hallberg, R., 1997. Stable split time stepping schemes for large-scale ocean modeling. J. Comp. Phys. 135(1), 54-65.

Hecht, M.W., 2010. Cautionary tales of persistent accumulation of numerical error: Dispersive centered advection. Ocean Modell. 35(3), 270-276.

Hirt, C., 1968. Heuristic stability theory for finite-difference equations. J. Comp. Phys. 2(4), 339 - 355. Hundsdorfer, W., Trompert, R.A., 1994. Method of lines and direct discretization: A comparison for linear 
advection. Appl. Numer. Math. 13(6), 469-490.

Ilicak, M., Adcroft, A.J., Griffies, S.M., Hallberg, R.W., 2012. Spurious dianeutral mixing and the role of momentum closure. Ocean Modell. 45-46, 37-58.

Jackett, D.R., McDougall, T.J., 1995. Minimal adjustment of hydrographic profiles to achieve static stability. J. Atmos. Ocean. Technol. 12(2), 381-389.

Karni, S., 1994. On the group velocity of symmetric and upwind numerical schemes. International Journal for Numerical Methods in Fluids 18(11), 1073-1081.

Lauritzen, P.H., Ullrich, P.A., Jablonowski, C., Bosler, P.A., Calhoun, D., Conley, A.J., Enomoto, T., Dong, L., Dubey, S., Guba, O., Hansen, A.B., Kaas, E., Kent, J., Lamarque, J.F., Prather, M.J., Reinert, D., Shashkin, V.V., Skamarock, W.C., Sørensen, B., Taylor, M.A., Tolstykh, M.A., 2014. A standard test case suite for two-dimensional linear transport on the sphere: results from a collection of state-of-the-art schemes. Geoscientific Model Development 7(1), 105-145.

Lax, P., Wendroff, B., 1960. Systems of conservation laws. Communications on Pure and Applied Mathematics 13(2), 217-237.

Lazure, P., Dumas, F., 2008. An external-internal mode coupling for a 3d hydrodynamical model for applications at regional scale (MARS). Advances in Water Resources 31(2), 233 - 250.

Leclair, M., Madec, G., 2011. ż-coordinate, an arbitrary lagrangian-eulerian coordinate separating high and low frequency motions. Ocean Modell. 37(3-4), 139 - 152.

Lecointre, A., Molines, J.M., Barnier, B., 2011. Definition of the interannual experiment ORCA12.L46-MAL95, 1989-2007. Technical Report. LEGI-DRA-21-10-2011. Available online at http: //www.drakkar-ocean.eu/publications/reports/rapport-run-orcal2. $146-\operatorname{mal} 95 . p d f$.

Lele, S.K., 1992. Compact finite difference schemes with spectral-like resolution. J. Comp. Phys. 103(1), $16-42$.

Lemarié, F., Debreu, L., Shchepetkin, A., McWilliams, J., 2012a. On the stability and accuracy of the harmonic and biharmonic isoneutral mixing operators in ocean models. Ocean Modell. 52-53, 9-35.

Lemarié, F., Kurian, J., Shchepetkin, A.F., Molemaker, M.J., Colas, F., McWilliams, J.C., 2012b. Are There Inescapable Issues Prohibiting the use of Terrain-Following Coordinates in Climate Models ? Ocean Modell. 42, 57-79.

Leonard, B.P., 1979. A stable and accurate convective modeling procedure based on quadratic upstream interpolation. Computer Methods in Applied Mechanics and Engineering 19, 59-98.

Leonard, B.P., Lock, A.P., McVean, M., 1996. Conservative explicit unrestricted-time-step constancy preserving advection schemes. Mon. Weather Rev. 124, 2588-2606.

Lévy, M., Estublier, A., Madec, G., 2001. Choice of an advection scheme for biogeochemical models. Geophys. Res. Lett. 28(19), 3725-3728.

Madec, G., 2008. NEMO ocean engine, in: Note du Pole de modélisation No. 27. Institut Pierre-Simon Laplace (IPSL), France.

Madec, G., Chartier, M., Delécluse, P., Crépon, M., 1991. A three-dimensional numerical study of deep water formation in the Northwestern Mediterranean Sea. J. Phys. Oceanogr. 21(9), 1349-1371.

Marchesiello, P., Capet, X., Menkes, C., Kennan, S., 2011. Submesoscale dynamics in Tropical Instability Waves. Ocean Modell. 39, 31-46.

Molines, J.M., Barnier, B., Penduff, T., Brodeau, L., Treguier, A., Theetten, S., Madec, G., 2007. Definition of the global $1 / 2^{\circ}$ experiment with CORE forcing, ORCA05-G50. Technical Report. LEGI-DRA-111-2006. Available online at http://www.drakkar-ocean.eu/publications/reports / orca05-g50.pdf. 
Orszag, S.A., Israeli, M., 1974. Numerical simulations of viscous incompressible flows. Annu. Rev. Fluid Mech. 6, 281-318.

Sadourny, R., 1975. The dynamics of finite-difference models of the shallow-water equations. J. Atmos. Sci. 32(4), 680-689.

Sescu, A., Afjeh, A., Hixon, R., Sescu, C., 2010. Conditionally stable multidimensional schemes for advective equations. J. Sci. Comput. 42(1), 96-117.

Sescu, A., Hixon, R., Afjeh, A.A., 2008. Multidimensional optimization of finite difference schemes for computational aeroacoustics. J. Comp. Phys. 227(9), 4563 - 4588.

Shchepetkin, A.F., 2015. An adaptive, courant-number-dependent implicit scheme for vertical advection in oceanic modeling. Ocean Modell. Doi:10.1016/j.ocemod.2015.03.006.

Shchepetkin, A.F., McWilliams, J.C., 2005. The Regional Oceanic Modeling System: A split-explicit, free-surface, topography-following-coordinate ocean model. Ocean Modell. 9(4), 347-404.

Shchepetkin, A.F., McWilliams, J.C., 2008. Computational kernel algorithms for fine-scale, multiprocess, longtime oceanic simulations, in: Ciarlet, P.G. (Ed.), Computational methods for the atmosphere and the oceans Special Volume. Elsevier. volume XIV of Handbook of numerical analysis.

Shchepetkin, A.F., McWilliams, J.C., 2011. Accurate Boussinesq oceanic modeling with a practical, "Stiffened" Equation of State. Ocean Modell. 38(1-2), 41-70.

Shchepetkin, A.F., Molemaker, M.J., Colas, F., Mason, E., Uchiyama, Y., Kurian, J., McWilliams, J.C., 2009. Recent Developments of ROMS at UCLA. Oceanography Review Symposium Chicago, IL, June 7-13 Available online at http://www.atmos.ucla.edu/ alex/ROMS/ Chicago2009Talk.pdf.

Shukla, R.K., Giri, P., 2014. Isotropic finite volume discretization. J. Comp. Phys. In press.

Skamarock, W.C., 2011. Kinetic energy spectra and model filters, in: Lauritzen, P., Jablonowski, C., Taylor, M., Nair, R. (Eds.), Numerical Techniques for Global Atmospheric Models. Springer Berlin Heidelberg. volume 80 of Lecture Notes in Computational Science and Engineering, pp. 495-512.

Skamarock, W.C., Klemp, J.B., 2008. A time-split nonhydrostatic atmospheric model for weather research and forecasting applications. J. Comp. Phys. 227, 3465-3485.

Smith, R.D., Jones, P., Briegleb, B., Bryan, F., Danabasoglu, G., Dennis, J., Dukowicz, J., Eden, C., FoxKemper, B., Gent, P., Hecht, M., Jayne, S., Jochum, M., Large, W., Lindsay, K., Maltrud, M., Norton, N., Peacock, S., Vertenstein, M., Yeager, S., 2010. The Parallel Ocean Program (POP) Reference Manual, 23 March 2010, LAUR-10-01853.

Soufflet, Y., Marchesiello, P., Jouanno, J., Capet, X., Debreu, L., Lemarié, F., 2015. On effective resolution in ocean models. Ocean Modell. Submitted.

Strang, G., 1968. On the construction and comparison of difference schemes. SIAM Journal on Numerical Analysis 5(3), 506-517. http://epubs.siam.org/doi/pdf/10.1137/0705041.

Sun, W.Y., Sun, O.M., 2011. A modified leapfrog scheme for shallow water equations. Computers \& Fluids 52(0), $69-72$.

Suresh, A., Huynh, H., 1997. Accurate monotonicity-preserving schemes with rungekutta time stepping. J. Comp. Phys. 136(1), 83 - 99.

Thuburn, J., Kent, J., Wood, N., 2014. Cascades, backscatter and conservation in numerical models of two-dimensional turbulence. Quart. J. Roy. Meteorol. Soc. 140(679), 626-638.

Wallcraft, A.J., 2011. New Features of HYCOM. 15th Layered Ocean Model Workshop Available online at http://coaps.fsu.edu/LOM/2011/pdf/026_talk_Alan_hycom_11a.pdf.

Walters, R.A., Lane, E.M., Hanert, E., 2009. Useful time-stepping methods for the coriolis term in a shallow water model. Ocean Modell. 28(13), 66 - 74. The Sixth International Workshop on Unstructured 
Mesh Numerical Modelling of Coastal, Shelf and Ocean Flows.

Winther, N.G., Morel, Y.G., Evensen, G., 2007. Efficiency of high order numerical schemes for momentum advection. J. Mar. Res. 67(12), 31 - 46.

Yamaguchi, T., Randall, D.A., Khairoutdinov, M.F., 2011. Cloud modeling tests of the ULTIMATEMACHO scalar advection scheme. Mon. Weather Rev. 139, 3248 - 3264.

Zalesak, S.T., 1979. Fully multidimensional flux-corrected transport algorithms for fluids. J. Comp. Phys. 31(3), $335-362$. 


\section{A Numerical solution of the eigenvalue problem (1.4)}

The squared buoyancy frequency profile $N^{2}(z)$ is estimated at cell interfaces $z=z_{k+\frac{1}{2}}$ using cellcentered temperature and salinity data. We use the formulation of the equation of state (EOS) described in Shchepetkin and McWilliams (2011). This EOS form is based on a Taylor expansion of the EOS proposed in Jackett and McDougall (1995), leading to

$$
\rho=\rho_{1}^{(0)}+\rho_{1}^{\prime}(\vartheta, S)+q_{1}^{\prime}(\vartheta, S) \cdot z(1-\eta z),
$$

where $\rho_{1}^{(0)}+\rho_{1}^{\prime}(\vartheta, S)$ is the sea-water density at the standard pressure of 1 Atm (sea surface), $\vartheta$ is the potential temperature, $\mathrm{S}$ the salinity, and the $q_{1}^{\prime}$ term represents a compressibility coefficient. $q_{1}^{\prime}(\theta, S)$ is given by equation (4.6) in Shchepetkin and McWilliams (2011) and $\eta=1.72 \times 10^{-5} \mathrm{~m}^{-1}$. The resulting formulation for $N^{2}\left(z_{k+\frac{1}{2}}\right)$ based on neutral density gradients is thus

$$
N^{2}\left(z_{k+\frac{1}{2}}\right)=-\frac{g}{\rho_{0}}\left\{\rho_{1 i, j, k+1}^{\prime}-\rho_{1 i, j, k}^{\prime}+\left(q_{1 i, j, k+1}^{\prime}-q_{1 i, j, k}^{\prime}\right) \frac{z_{i, j, k+1}+z_{i, j, k}}{2}\left[1-\eta \frac{z_{i, j, k+1}+z_{i, j, k}}{2}\right]\right\}
$$

with boundary conditions $N^{2}\left(z_{N+\frac{1}{2}}\right)=N^{2}\left(z_{N-\frac{1}{2}}\right)$ at the surface and $N^{2}\left(z_{\frac{1}{2}}\right)=N^{2}\left(z_{\frac{3}{2}}\right)$ at the bottom. In (A.1), negative values of $N^{2}(z)$ correspond to stable stratifications. Two different ways of handling unstratified and unstable regions have been investigated :

(1) replacing the value of $N^{2}(z)$ by $-\epsilon$ when $N^{2}(z) \geq-\epsilon$. Values of $\epsilon$ between $10^{-8}$ and $10^{-14}$ have been tested.

(2) leaving unstable profiles and poorly stratified regions. In this case, negative eigenvalues are obtained but are discarded from the sorting procedure to recover the phase speed of the first baroclinic mode.

Our results showed very modest sensitivity to the way the unstratified regions are handled (below $5 \%$ of relative change), and method (1) with $\epsilon=10^{-10}$ has been used.

\section{B Derivation of a 4th-order Space-time Compact Scheme (Co4st)}

Let us consider the one-dimensional vertical advection equation

$$
\partial_{t} q+w_{0} \partial_{z} q=0
$$

with $w_{0}$ a constant vertical velocity. The approximation of the vertical derivative at $z=z_{k}$ is naturally given by

$$
\left.\frac{\partial q}{\partial z}\right|_{z=z_{k}}=\frac{\widetilde{q}_{k+\frac{1}{2}}-\widetilde{q}_{k-\frac{1}{2}}}{\Delta z}
$$

where the $\widetilde{q}$ interfacial values are unknown.

Derivation of the classical fourth-order compact scheme (Co4)

Assuming a standard second order centered scheme, the following equality is obtained 


$$
\left.\frac{\partial q}{\partial z}\right|_{z=z_{k}}=\frac{q_{k+1}-q_{k-1}}{2 \Delta z}-\frac{\Delta z^{2}}{6} \partial_{z}^{3} q+\mathscr{O}\left(\Delta z^{4}\right) \approx D_{\mathrm{C} 2}\left\{\left(1-\frac{\Delta z^{2}}{6} \partial_{z}^{2}\right) q_{k}\right\}+\mathscr{O}\left(\Delta z^{4}\right)
$$

where $D_{\mathrm{C} 2}\left\{p_{k}\right\}=\frac{p_{k+1}-p_{k-1}}{2 \Delta z}$. Indeed, fourth-order accuracy requires the discretization of the righthand-side term $\frac{\Delta z^{2}}{6} \partial_{z}^{3} q$. Classical discretization is given by

$$
D_{\mathrm{C} 2}\left\{\left.\frac{\Delta z^{2}}{6} \partial_{z}^{2} q\right|_{z=z_{k}}\right\}=\frac{1}{12 \Delta z}\left(\delta^{2} q_{k+1}-\delta^{2} q_{k-1}\right)+\mathscr{O}\left(\Delta z^{4}\right), \quad \delta^{2} q_{k+1}=q_{k+2}-2 q_{k+1}+q_{k},
$$

which extends the stencil to points $q_{k+2}$ and $q_{k-2}$. Following Orszag and Israeli (1974) (see also Lele, 1992), it is possible to achieve fourth-order accuracy with more compact stencil (i.e. on the 3-point stencil typically used for the standard second-order algorithms) using a Padé approximation of the term $1-\frac{\Delta z^{2}}{6} \partial_{z}^{2}$ in (B.1)

$$
1-\frac{\Delta z^{2}}{6} \partial_{z}^{2}=\frac{1}{1+\frac{\Delta z^{2}}{6} \partial_{z}^{2}}+\mathscr{O}\left(\Delta z^{4}\right)
$$

which leads to an implicit discretization. Indeed, after substitution in (B.1), we get

$$
\left.\left(1+\frac{\delta^{2}}{6}\right) \frac{\partial q}{\partial z}\right|_{z=z_{k}}=\left.\frac{1}{6} \frac{\partial q}{\partial z}\right|_{z=z_{k+1}}+\left.\frac{2}{3} \frac{\partial q}{\partial z}\right|_{z=z_{k}}+\left.\frac{1}{6} \frac{\partial q}{\partial z}\right|_{z=z_{k-1}}=D_{\mathrm{C} 2}\left\{q_{k}\right\}+\mathscr{O}\left(\Delta z^{4}\right)
$$

which leads to

$$
\frac{1}{6} \widetilde{q}_{k+\frac{3}{2}}+\frac{2}{3} \widetilde{q}_{k+\frac{1}{2}}+\frac{1}{6} \widetilde{q}_{k-\frac{1}{2}}=\frac{q_{k}+q_{k+1}}{2} .
$$

The unknowns $\widetilde{q}$ are thus computed as the solution of a tridiagonal system.

\section{Coupled space-time fourth-order compact scheme (Co4st)}

To derive a coupled space-time version of previous scheme, we proceed in a slightly different way. From (B.2), we can remark that the interfacial values $\widetilde{q}$ are solution of a tridiagonal system

$$
a_{+} \widetilde{q}_{k+\frac{3}{2}}+\widetilde{q}_{k+\frac{1}{2}}+a_{-} \widetilde{q}_{k-\frac{1}{2}}=\tau\left(q_{k}+q_{k+1}\right) .
$$

The coefficients $a_{+}, a_{-}$and $\tau$ are chosen to reach the target accuracy. Considering a Fourier component such that $q_{k}=e^{i \theta k}$ (for simplicity we consider that the amplitude/phase of the corresponding component is 1 ), with $\theta=k_{z} \Delta z$, we get

$$
\widetilde{q}_{k+\frac{1}{2}}=\frac{\tau\left(e^{i \frac{\theta}{2}}+e^{-i \frac{\theta}{2}}\right)}{1+a_{+} e^{i \theta}+a_{-} e^{-i \theta}} \cdot e^{i \theta\left(k+\frac{1}{2}\right)}=\frac{2 \tau \cos \theta / 2}{1+\left(a_{+}+a_{-}\right) \cos \theta+i\left(a_{+}-a_{-}\right) \sin \theta} \cdot e^{i \theta\left(k+\frac{1}{2}\right)}
$$

therefore 


$$
\left.\Delta z \frac{\partial q}{\partial z}\right|_{z=z_{k}}=\widetilde{q}_{k+\frac{1}{2}}-\widetilde{q}_{k-\frac{1}{2}}=\frac{2 i \tau \sin \theta}{1+\left(a_{+}+a_{-}\right) \cos \theta+i\left(a_{+}-a_{-}\right) \sin \theta} \cdot e^{i k \theta}=\mathscr{S}_{\mathrm{Co} 4}(\theta) \cdot e^{i k \theta}
$$

Taylor expansion leads to

$$
\mathscr{S}_{\mathrm{Co} 4}(\theta) \approx i \theta\left(\frac{2 \tau}{\left(1+a_{+}+a_{-}\right)}-\frac{2\left(a_{+}-a_{-}\right) i \theta}{\left(1+a_{+}+a_{-}\right)^{2}}-\frac{\left(1-a_{+}-a_{-}+4\left(a_{-}^{2}+a_{+}^{2}\right)-16 a_{-} a_{+}\right) \theta^{2}}{3\left(1+a_{+}+a_{-}\right)^{2}}\right)+\mathscr{O}\left(\theta^{4}\right)
$$

Fourth order accuracy in space is thus obtained for $2 \tau=1+a_{+}+a_{-}, a_{+}=a_{-}$, and $1-a_{+}-a_{-}+4\left(a_{-}^{2}+\right.$ $\left.a_{+}^{2}\right)=16 a_{-} a_{+}$, which leads to $a_{+}=a_{-}=1 / 4$ and $\tau=3 / 4$ (those coefficients are consistent with (B.2)).

Let us now introduce the time dimension in the problem using a simple one-step explicit Euler scheme

$$
q_{k}^{n+1}=\left(1-\alpha_{\mathrm{adv}} \mathscr{S}_{\mathrm{Co} 4}(\theta)\right) q_{k}^{n}, \quad \alpha_{\mathrm{adv}}=\frac{w_{0} \Delta t}{\Delta z} .
$$

The physical mode associated to this scheme is simply $\lambda\left(\theta, \alpha_{\text {adv }}\right)=1-\alpha_{\text {adv }} \mathscr{S}_{\text {Co4 }}(\theta)$. Fourth-order accuracy in space-time is obtained for

$$
\lambda\left(\theta, \alpha_{\mathrm{adv}}\right)=1-i \alpha_{\mathrm{adv}} \theta-\frac{\left(\alpha_{\mathrm{adv}} \theta\right)^{2}}{2}+i \frac{\left(\alpha_{\mathrm{adv}} \theta\right)^{3}}{6}+\frac{\left(\alpha_{\mathrm{adv}} \theta\right)^{4}}{24}+\mathscr{O}\left(\theta^{5}\right)
$$

This equality is satisfied under the following constraints on the coefficients

$$
\left\{\begin{aligned}
2 \tau & =1+a_{+}+a_{-} \\
4 \tau\left(a_{+}-a_{-}\right) & =\alpha_{\mathrm{adv}}\left(1+a_{+}+a_{-}\right)^{2} \\
6 \tau\left(1-a_{+}-a_{-}+4\left(a_{-}^{2}+a_{+}^{2}\right)-16 a_{-} a_{+}\right) & =3 \alpha_{\mathrm{adv}}^{2}\left(1+a_{+}+a_{-}\right)^{3} \\
48 \tau\left(a_{+}-a_{-}\right)\left(1+a_{+}^{2}+a_{-}^{2}-a_{+}-a_{-}-10 a_{+} a_{-}\right) & =3 \alpha_{\mathrm{adv}}^{3}\left(1+a_{+}+a_{-}\right)^{4}
\end{aligned}\right.
$$

We obtain the following coefficients

$$
a_{+}=-\frac{\alpha_{\mathrm{adv}}+1}{2\left(\alpha_{\mathrm{adv}}-2\right)}, \quad a_{-}=-\frac{\alpha_{\mathrm{adv}}-1}{2\left(\alpha_{\mathrm{adv}}+2\right)}, \quad \tau=-\frac{3}{\left(\alpha_{\mathrm{adv}}+2\right)\left(\alpha_{\mathrm{adv}}-2\right)},
$$

and the scheme reads

$$
\left(\alpha_{\mathrm{adv}}+1\right)\left(\alpha_{\mathrm{adv}}+2\right) \widetilde{q}_{k+\frac{3}{2}}+2\left(\alpha_{\mathrm{adv}}+2\right)\left(2-\alpha_{\mathrm{adv}}\right) \widetilde{q}_{k+\frac{1}{2}}+\left(\alpha_{\mathrm{adv}}-1\right)\left(\alpha_{\mathrm{adv}}-2\right) \widetilde{q}_{k-\frac{1}{2}}=6\left(q_{k}+q_{k+1}\right) .
$$

The resulting scheme is unconditionally stable (with neutral stability, i.e. $\left|\lambda\left(\theta, \alpha_{\text {adv }}\right)\right|=1, \forall \theta \in[0, \pi]$ and $\forall \alpha_{\text {adv }}$ ) and it is exact for $\alpha_{\text {adv }}=1$ and $\alpha_{\text {adv }}=2$. Phase errors are plotted in Fig. B.1. The coupled space-time scheme shows very good accuracy in terms of phase speed for scales larger or equal to $4 \Delta x$. Unlike most implicit schemes (e.g. Shchepetkin, 2015, Sec. 2.2) the Co4st scheme behaves well for small and large Courant numbers. It is worth noting that the tridiagonal matrix associated to (B.3) is not always diagonally-dominant. Therefore, standard Gaussian elimination procedure can not be used and cyclic reduction algorithm should be preferred. 


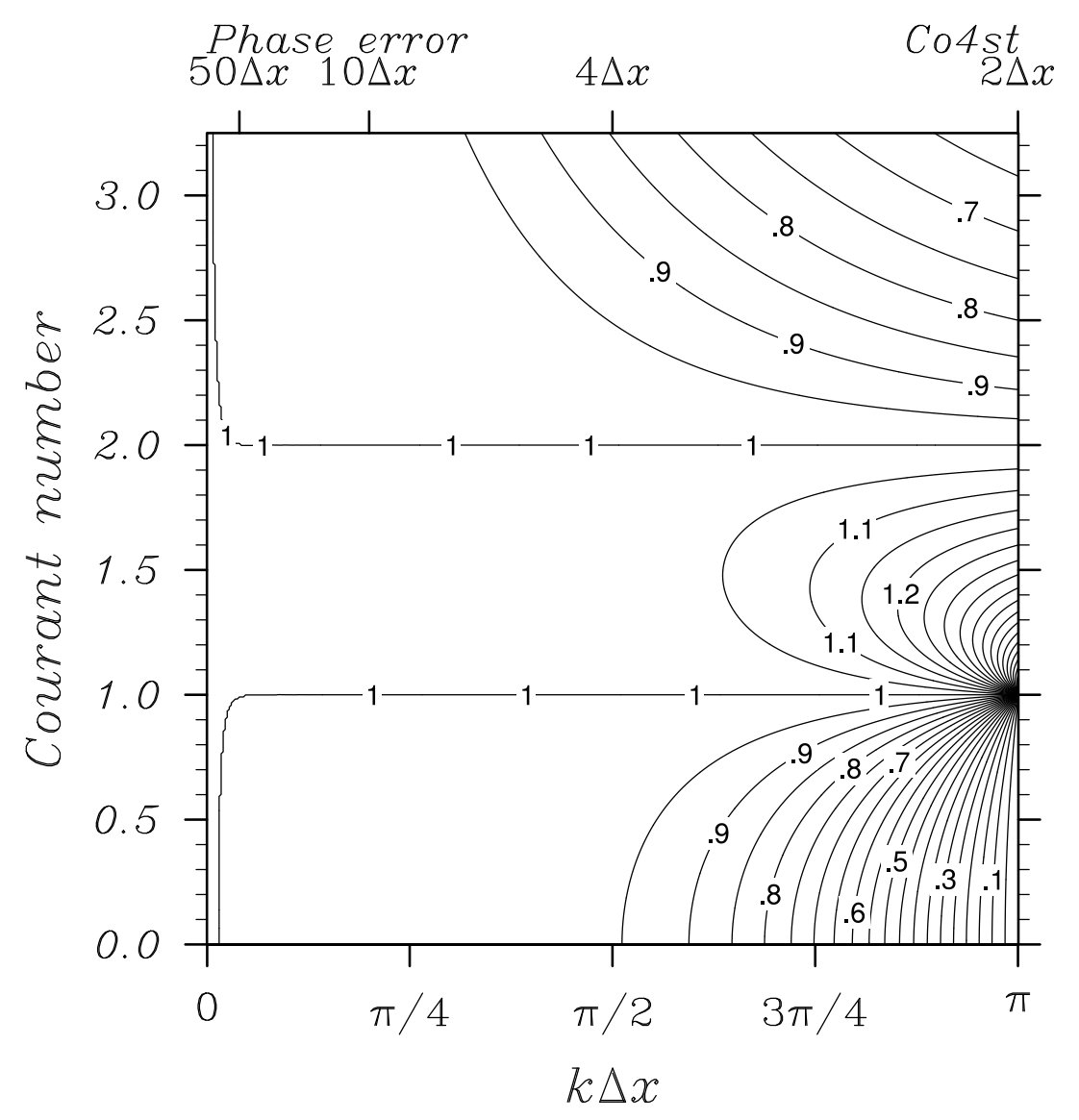

Fig. B.1. Phase error $-\frac{\arg \lambda\left(\theta, \alpha_{\text {adv }}\right)}{\theta \alpha_{\text {adv }}}$ (with $\alpha_{\text {adv }}$ the Courant number, $\theta$ the normalized wavenumber, and $\lambda$ the amplification factor) with respect to the Courant number and the normalized wavenumber $k \Delta x$ for the fourth-order coupled space-time compact scheme (Co4st).

\section{Time and Space Discretizations of the Linear Advection Equation}

\section{C.1 Uncoupled Space-Time Approach}

We describe here the space-time discretizations discussed in the paper and representative of the current practice in state-of-the-art oceanic models. A simple one-dimensional linear advection equation with velocity $u_{0}$ is considered. The schemes under consideration are straightforward to extend to multi-dimensions because they do not require transverse terms in the advective flux computations to circumvent the computational instability associated with directional flux-splitting in multi-dimensions (Leonard et al., 1996). Those additional terms are only required in the case of one-step space-time methods, as discussed in Sec. C.2.

We consider the third-order upwind scheme (UP3) in space, the corresponding scheme reads

$$
\widehat{q}_{i-\frac{1}{2}}^{\mathrm{up} 3}=\widehat{q}_{i-\frac{1}{2}}^{\mathrm{c} 4}-\operatorname{sign}\left(1, u_{0}\right) \frac{q_{i-2}-3 q_{i-1}+3 q_{i}-q_{i+1}}{12}, \quad \widehat{q}_{i-\frac{1}{2}}^{\mathrm{c} 4}=\frac{-q_{i-2}+7 q_{i-1}+7 q_{i}-q_{i+1}}{12} .
$$

(C.1) clearly shows that a third-order upwind scheme is equivalent to a fourth-order centered scheme 
plus an explicit biharmonic operator with flow dependent hyperdiffusivity. A first temporal scheme under consideration is the Leapfrog Adams-Moulton predictor-corrector scheme (LFAM3) (Shchepetkin and McWilliams, 2005). This scheme reads

$$
\left\{\begin{array}{c}
q_{i}^{n+1, \star}=q_{i}^{n-1}-2 \alpha_{\text {adv }}\left\{\left(\widehat{q}_{i+\frac{1}{2}}^{\text {up } 3}\right)^{n}-\left(\widehat{q}_{i-\frac{1}{2}}^{\text {up } 3}\right)^{n}\right\} \\
q_{i}^{n+\frac{1}{2}}=5 / 12 q_{i}^{n+1, \star}+2 / 3 q_{i}^{n}-1 / 12 q_{i}^{n-1} \\
q_{i}^{n+1}=q_{i}^{n}-\alpha_{\text {adv }}\left\{\left(\widehat{q}_{i+\frac{1}{2}}\right)^{n+\frac{1}{2}}-\left(\widehat{q}_{i-\frac{1}{2}}^{\text {up } 3}\right)^{n+\frac{1}{2}}\right\}
\end{array}\right.
$$

with $\alpha_{\text {adv }}=u_{0} \Delta t / \Delta x$ the Courant number. An other option is the second-order Adams-Bashforth method $(\mathrm{AB} 2 \varepsilon)$. This scheme is based on an extrapolation in time at time $n+\frac{1}{2}+\varepsilon$ followed by a correction step

$$
\left\{\begin{array}{l}
q_{i}^{n+\frac{1}{2}}=(3 / 2+\varepsilon) q_{i}^{n}-(1 / 2+\varepsilon) q_{i}^{n-1} \\
q_{i}^{n+1}=q_{i}^{n}-\alpha_{\text {adv }}\left\{\left(\widehat{q}_{i+\frac{1}{2}}^{\text {up3 }}\right)^{n+\frac{1}{2}}-\left(\widehat{q}_{i-\frac{1}{2}}^{\text {up3 }}\right)^{n+\frac{1}{2}}\right\}
\end{array}\right.
$$

(corrector).

The parameter $\varepsilon$ is necessary to control an instability of the scheme when the extrapolation step is done at time $n+1 / 2$ (e.g. Adcroft et al., 2014). A typical value for this parameter, as used for practical applications, is $\varepsilon=0.1$. The extension to third-order accuracy of the Adams-Bashforth approach (AB3) is

$$
\begin{cases}q_{i}^{n+\frac{1}{2}}=(23 / 12) q_{i}^{n}-(16 / 12) q_{i}^{n-1}+(5 / 12) q_{i}^{n-2} & \text { (AB3) } \\ q_{i}^{n+1}=q_{i}^{n}-\alpha_{\text {adv }}\left\{\left(\widehat{q}_{i+\frac{1}{2}}^{\operatorname{up} 3}\right)^{n+\frac{1}{2}}-\left(\widehat{q}_{i-\frac{1}{2}}^{\text {up3 }}\right)^{n+\frac{1}{2}}\right\} & \text { (corrector) }\end{cases}
$$

which requires an extra storage of $q$ at time $n-2$.

In Skamarock and Klemp (2008), a third-order Runge-Kutta (RK3) is presented

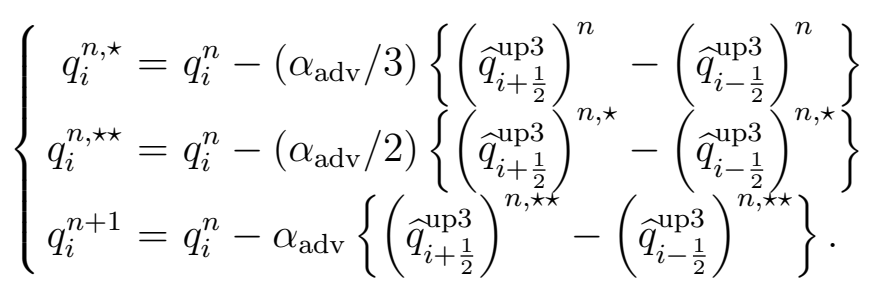

This RK3 variant is third-order accurate in time for linear equations, but becomes second-order for nonlinear terms (Baldauf, 2008).

The schemes introduced so far are all conditionally stable for advective and diffusive processes (Tab. 2). For the Leapfrog (LF) scheme, it is well known that this scheme is unconditionally unstable for diffusive terms. In order to achieve a stable integration of the third-order upwind scheme, the flux $\widehat{q}_{i-\frac{1}{2}}^{\text {up } 3}$ in (C.1) is split between its centered part $\widehat{q}_{i-\frac{1}{2}}^{\mathrm{c}}$ advanced using the LF scheme and the diffusive part advanced using an Euler scheme over $2 \Delta t$. The resulting scheme, combined with a Robert-Asselin (RA) filter, reads

$$
\left\{\begin{aligned}
q_{i}^{n+1, \star} & =q_{i}^{n-1}-2 \alpha_{\mathrm{adv}}\left\{\left(\widehat{q}_{i+\frac{1}{2}}^{\mathrm{c} 4}\right)^{n, \star}-\left(\widehat{q}_{i-\frac{1}{2}}^{\mathrm{c} 4}\right)^{n, \star}\right\}-\frac{\left|\alpha_{\mathrm{adv}}\right|}{6}\left(-q_{i-2}^{n-1}+4 q_{i-1}^{n-1}-6 q_{i}^{n-1}+4 q_{i+1}^{n-1}-q_{i+2}^{n-1}\right) \\
q_{i}^{n} & =\nu q_{i}^{n+1, \star}+(1-2 \nu) q^{n, \star}+\nu q_{i}^{n-1} .
\end{aligned}\right.
$$


We do not consider alternatives to the usual Robert-Asselin filter because none of them had significant impact on the phase and amplification errors when dissipation is added to the problem. Indeed, those alternatives were derived in the non-dissipative case only, which is not representative of the general functioning of realistic models. Moreover, most of them had negative feedback on the stability of the scheme.

\section{C.2 Coupled Space-Time approach and Directional Splitting}

An attractive approach for oceanic models is to use high-order one-step space-time schemes built on the extension of the Lax-Wendroff methodology to higher-order (e.g.; Hundsdorfer and Trompert, 1994; Daru and Tenaud, 2004). If space and time discretizations are separated, as usually done in oceanic models, the resulting scheme requires large spatial stencils and multi-step time integration to reach high-order accuracy. On the other hand, coupled space-time are simple high-order one-step methods with compact stencil.

They are based on the assumption that time and space derivatives can be substituted for each other to cancel terms in the truncation error in time by additional terms in space. An example of such a scheme which is third-order in space and time is the Euler-QUICKEST scheme (QK3; Leonard, 1979)

$$
q_{i}^{n+1}=q_{i}^{n}-\alpha_{\text {adv }}\left\{\left(\widehat{q}_{i+\frac{1}{2}}^{\mathrm{qk} 3}\right)^{n}-\left(\widehat{q}_{i-\frac{1}{2}}^{\mathrm{qk} 3}\right)^{n}\right\}
$$

where the $\alpha_{\mathrm{adv}}$-dependent fluxes at cell-interfaces are given by

$\widehat{q}_{i-\frac{1}{2}}^{\mathrm{gk} 3}=\widehat{q}_{i-\frac{1}{2}}^{\mathrm{c} 2}-\frac{\alpha_{\mathrm{adv}}}{2}\left(q_{i}-q_{i-1}\right)+\frac{\alpha_{\mathrm{adv}}^{2}-1}{12}\left[\left(q_{i+1}-q_{i}-q_{i-1}+q_{i-2}\right)-\operatorname{sign}\left(1, u_{0}\right)\left(-q_{i-2}+3 q_{i-1}-3 q_{i}+q_{i+1}\right)\right]$

with $\widehat{q}_{i-\frac{1}{2}}^{\mathrm{c} 2}=\left(q_{i}+q_{i-1}\right) / 2$. Unlike the schemes previously introduced, the extension of (C.7) to multidimensions is not straightforward. A simple application of the 1D fluxes in each spatial direction would result in a scheme that can be at most 1st-order accurate, whatever the order of accuracy of the 1D fluxes, and the scheme would be unconditionally unstable. Indeed, the truncation error would be dominated by transverse terms corresponding to the contribution of cross-derivatives. To overcome this issue a directional splitting à la Strang (1968) can be used to introduce the necessary transverse terms in the discretization. However, for a constant tracer $q_{i, j, k} \equiv 1$ this approach would produce a spurious term of the form $\frac{\Delta t^{2}}{\Delta x \Delta y} \partial_{y}\left(v \partial_{x} u\right)$, therefore it would fail to satisfy the constancy preservation property of the continuous problem. The simplest method to introduce transverse terms while preserving constancy is the splitting of Bott (2010)

$$
\left\{\begin{aligned}
q_{i, j}^{\star} & =q_{i, j}^{n}-\Delta t\left(\partial_{x}\left(u q^{n}\right)-q_{i, j}^{n} \partial_{x} u\right) \\
q_{i, j}^{\star \star} & =q_{i, j}^{\star}-\Delta t\left(\partial_{y}\left(v q^{\star}\right)-q_{i, j}^{n} \partial_{y} v\right) \\
q_{i, j}^{\star \star \star} & =q_{i, j}^{\star \star}-\Delta t\left(\partial_{z}\left(w q^{\star \star}\right)-q_{i, j}^{n} \partial_{y} w\right) \\
q_{i, j}^{n+1} & =q_{i, j}^{\star \star \star}
\end{aligned}\right.
$$

with $\mathbf{u}=(u, v, w)$ a three-dimensional non-divergent field. We can see here why the stability depends simply on the stability in each direction since the three stages must be independently stable for the multidimensional scheme to be stable. Splitting errors are minimized by alternating order of directions for each 
consecutive sub-step. An alternative is to use the Multidimensional Advective-Conservative Hybrid Operator (MACHO, Leonard et al., 1996). The description of the latter approach in three spatial dimensions with spatially variable velocities and scale factors can be found in Yamaguchi et al. (2011).

To our knowledge, the impact of the directional splitting in the context of oceanic flows has never been studied so far. Among other things, we expect difficulties to represent the evolution of terms involving both the zonal and meridional components (like vorticity and kinetic energy) because the two components are not integrated synchronously in time.

\section{Stability and Accuracy for Discretizations of the Linear Advection Equation}

For a very long time, the numerical design of advection in oceanic and atmospheric models was primarily chosen to conserve quadratic quantities like potential enstrophy or energy (Sadourny, 1975) in order to ensure nonlinear stability in the absence of viscous terms. This requirement has led to the use of the vector-invariant form of momentum equations and to discrete algorithms based on second-order centered schemes combined with Leapfrog (LF) time-stepping. In this context a popular way to control the LF computational mode is the Robert-Asselin time filter. Discretization schemes have thus been historically chosen for their ability to conform to conservation properties with less emphasis on accuracy. The latter is, however, key in enhancing the effective resolution of the solution for a given grid size (Marchesiello et al., 2011; Lauritzen et al., 2014). A difficulty is that the order of accuracy of a given scheme does not directly provide the order of accuracy of the amplitude and phase taken separately. Indeed, amplitude and phase errors represent the error in the modulus and the argument of the total error and those quantities may be computed more accurately than the general order of accuracy given by the truncation error. In this section, amplitude and group velocity errors are discussed, as well as the relationship between the truncation error and the stability of space-time discretization schemes. One of the aim of this appendix is to show that stability of a given space-time discretization does not necessarily involve that the corresponding scheme behaves properly. In the remainder we note $\alpha_{\mathrm{adv}}$ the Courant number and $\alpha_{\mathrm{adv}}^{\star}$ the CFL criterion.

\section{D.1 Error Analysis}

We can show that the truncation errors associated to the six schemes of interest read

$$
\begin{array}{lll}
\mathcal{E}_{\mathrm{LFRA}-\mathrm{UP} 3}=-\frac{\nu \Delta t}{4} \partial_{t}^{2} q+\left(\frac{1}{3}+\frac{\nu}{2}\right) \frac{\Delta t^{2}}{2} \partial_{t}^{3} q-\left(\frac{7 \nu \alpha_{\mathrm{adv}}^{3}}{48}+\frac{1}{12}\right)\left(u_{0} \Delta x^{3}\right) \partial_{x}^{4} q & +\mathcal{O}\left(\Delta t^{4}, \Delta x^{4}\right), \\
\mathcal{E}_{\mathrm{AB} 2-\mathrm{UP} 3}=-(\varepsilon \Delta t) \partial_{t}^{2} q+\left(\frac{5}{6}+\varepsilon\right) \frac{\Delta t^{2}}{2} \partial_{t}^{3} q-\left(\alpha_{\mathrm{adv}}^{3}\left(\frac{1}{4}+\varepsilon\right)+\frac{1}{12}\right)\left(u_{0} \Delta x^{3}\right) \partial_{x}^{4} q+\mathcal{O}\left(\Delta x^{4}, \Delta t^{4}\right), \\
\mathcal{E}_{\mathrm{LFAM} 3-\mathrm{UP} 3}=\frac{7 \alpha_{\mathrm{adv}}-6}{72}\left(u_{0} \Delta x^{3}\right) \partial_{x}^{4} q & +\mathcal{O}\left(\Delta x^{4}, \Delta t^{4}, \Delta x^{3} \Delta t\right), \\
\mathcal{E}_{\mathrm{RK} 3-\mathrm{UP} 3}=\frac{\alpha_{\mathrm{adv}}^{3}-2}{24}\left(u_{0} \Delta x^{3}\right) \partial_{x}^{4} q & +\mathcal{O}\left(\Delta x^{4}, \Delta t^{4}, \Delta x^{2} \Delta t^{2}\right), \\
\mathcal{E}_{\mathrm{AB} 3-\mathrm{UP} 3}=\frac{9 \alpha_{\mathrm{adv}}^{3}-2}{24}\left(u_{0} \Delta x^{3}\right) \partial_{x}^{4} q & +\mathcal{O}\left(\Delta x^{4}, \Delta t^{4}, \Delta x^{3} \Delta t\right), \\
\mathcal{E}_{\mathrm{QK} 3} & =\frac{\alpha_{\mathrm{adv}}+2 \alpha_{\mathrm{adv}}^{2}-2-\alpha_{\mathrm{adv}}^{3}}{24}\left(u_{0} \Delta x^{3}\right) \partial_{x}^{4} q & +\mathcal{O}\left(\Delta x^{4}, \Delta t^{4}, \Delta x^{2} \Delta t^{2}\right) .
\end{array}
$$



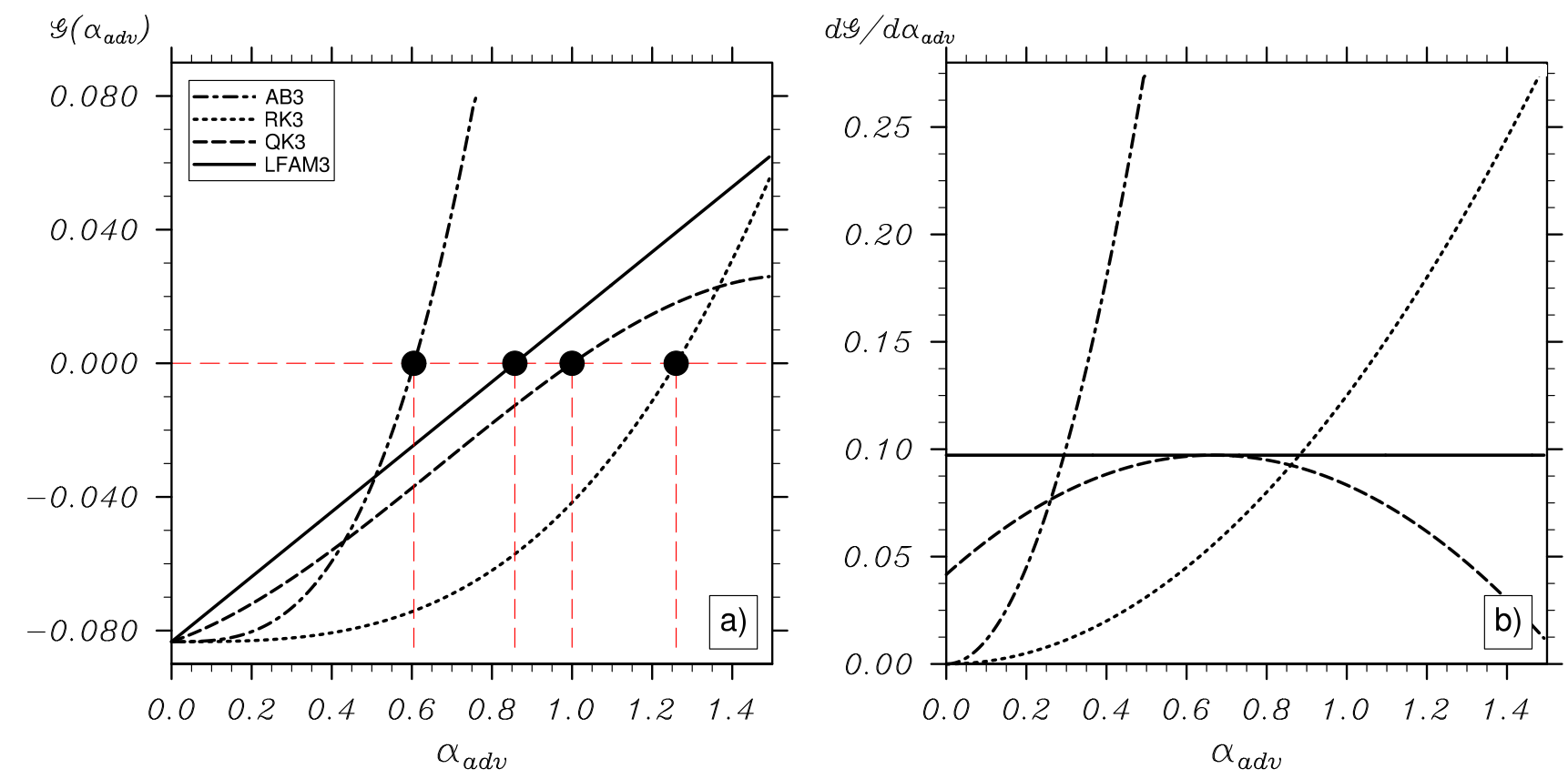

Fig. D.1. a) Evolution of the leading order term $\mathcal{G}$ of the space-time truncation error of the LFAM3-UP3, AB3-UP3, RK3-UP3 and QK3 schemes with respect to the Courant number $\alpha_{\text {adv }}$ in the case of a linear advection equation. b) Derivative of $\mathcal{G}$ with respect to $\alpha_{\text {adv }}$. On the left panel, red lines are drawn at places where $\mathcal{G}\left(\alpha_{\text {adv }}\right)=0$ for each space-time discretization.

To obtain those expressions we took advantage of the underlying PDE to replace time derivatives by space derivatives when relevant (indeed, $\partial_{t} q=-u_{0} \partial_{x} q$ ). The leading-order space-time truncation term of the RK3-UP3, AB3-UP3, LFAM3-UP3 and QK3 schemes can be written under the form $\mathcal{G}\left(\alpha_{\text {adv }}\right)\left(u_{0} \Delta x^{3}\right) \partial_{x}^{4} q$, with $\mathcal{G}$ a linear function of $\alpha_{\text {adv }}$, because those schemes are third-order accurate in space and time. In (D.1), the $7 \alpha_{\text {adv }} u_{0} \Delta x^{3} / 72$ term for the LFAM3-UP3 is associated to the time discretization error while the $u_{0} \Delta x^{3} / 12$ term is the contribution of the space discretization. In a similar way, the $\alpha_{\text {adv }}^{3} u_{0} \Delta x^{3} / 24$ term for RK3-UP3, $9 \alpha_{\mathrm{adv}}^{3} u_{0} \Delta x^{3} / 24$ for AB3-UP3, and $\left(\alpha_{\mathrm{adv}}+2 \alpha_{\mathrm{adv}}^{2}-\alpha_{\mathrm{adv}}^{3}\right) u_{0} \Delta x^{3} / 24$ for QK3 are linked to the discretization error in time. The leading order term for AB3 is exactly nine times larger than the one for RK3 however when considering those schemes combined with UP3 this conclusion does not hold anymore (Fig. D.1) and AB3-UP3 can have less error that RK3-UP3. Next we show how those space-time discretization errors can be useful to guess some features of discretization schemes.

\section{D.1.1 Stability}

The evolution of $\mathcal{G}$ with respect to the Courant number $\alpha_{\text {adv }}$ is shown in Fig. D.1, left panel. As long as LFAM3-UP3 is stable, the magnitude of its leading order error term is always smaller that of the QK3 and RK3 schemes. The LFAM3-UP3 scheme becomes 4 th order accurate for $\alpha_{\text {adv }}=0.857143$, very close to its stability limit $\alpha_{\mathrm{adv}}^{\star}=0.871$ (Tab. 2) which means that smaller Courant number would lead to larger discretization errors. Fig. D.1 also shows that QK3 is fourth order accurate for $\alpha_{\mathrm{adv}}^{\star}=1$ (in practice it turns out that QK3 becomes exact for $\alpha_{\mathrm{adv}}=1$ ) which is exactly its stability limit. In general, we see that when $\mathcal{G}$ is positive the scheme becomes unstable. This is not surprising as it means that there is a change of sign in front of the $\partial_{x}^{4} q$ term in the truncation error. This alternative to the Von-Neumann stability analysis based on truncation errors was previously suggested by Hirt (1968) for second-order space-time discretizations. Interestingly enough, this truncation error method for determining computational stability 

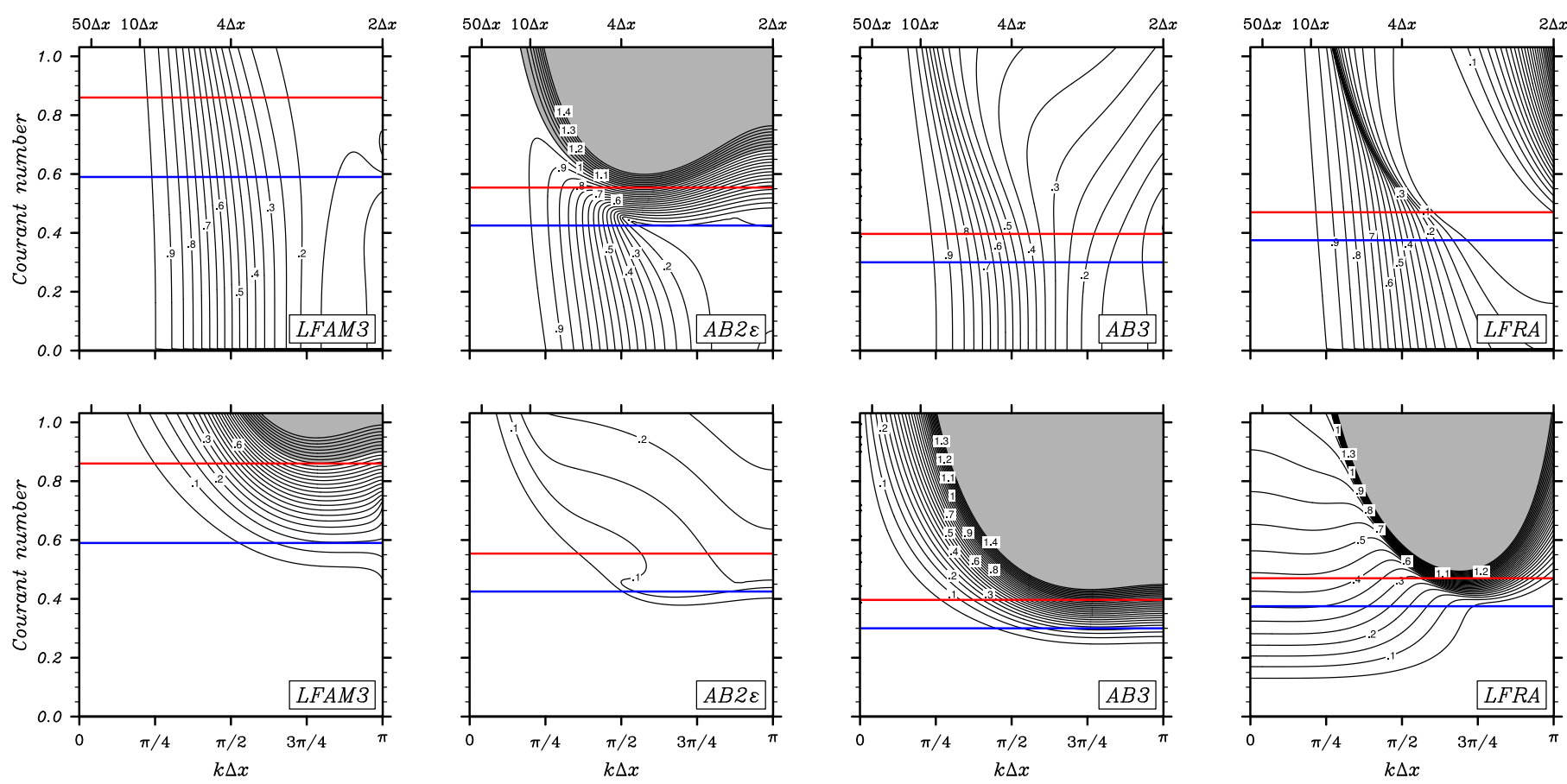

Fig. D.2. Maps of the amplification factor $|\lambda|^{\widetilde{\alpha} / \alpha}$ (with $\alpha$ the advective Courant number and $\widetilde{\alpha}=1.75$ ) with respect to the Courant number and the normalized wavenumber $k \Delta x$ for 4 different time-stepping algorithms (which are prone to a computational mode) combined with a third-order accurate space discretization. Results are shown for the LFAM3-UP3 (left), the AB2-UP3 (middle,left) scheme (with $\varepsilon=0.1$ ), the AB3-UP3 (middle,right) scheme, and the LFRA-UP3 (right) scheme (with $\nu=0.1$ ). The physical mode is represented in the top row and the computational mode in the bottom row. The solid red line represents the stability limit for each scheme and the solid blue line the limit for monotonic damping (for the physical mode). Gray shaded areas denote amplification factors larger than 1, i.e. unstable regions. The top $\mathrm{x}$-axis represents wavelengths. The acronyms are LFAM = Leapfrog Adams-Moulton, LFRA = Leapfrog Robert-Asselin, AB=Adams-Bashforth.
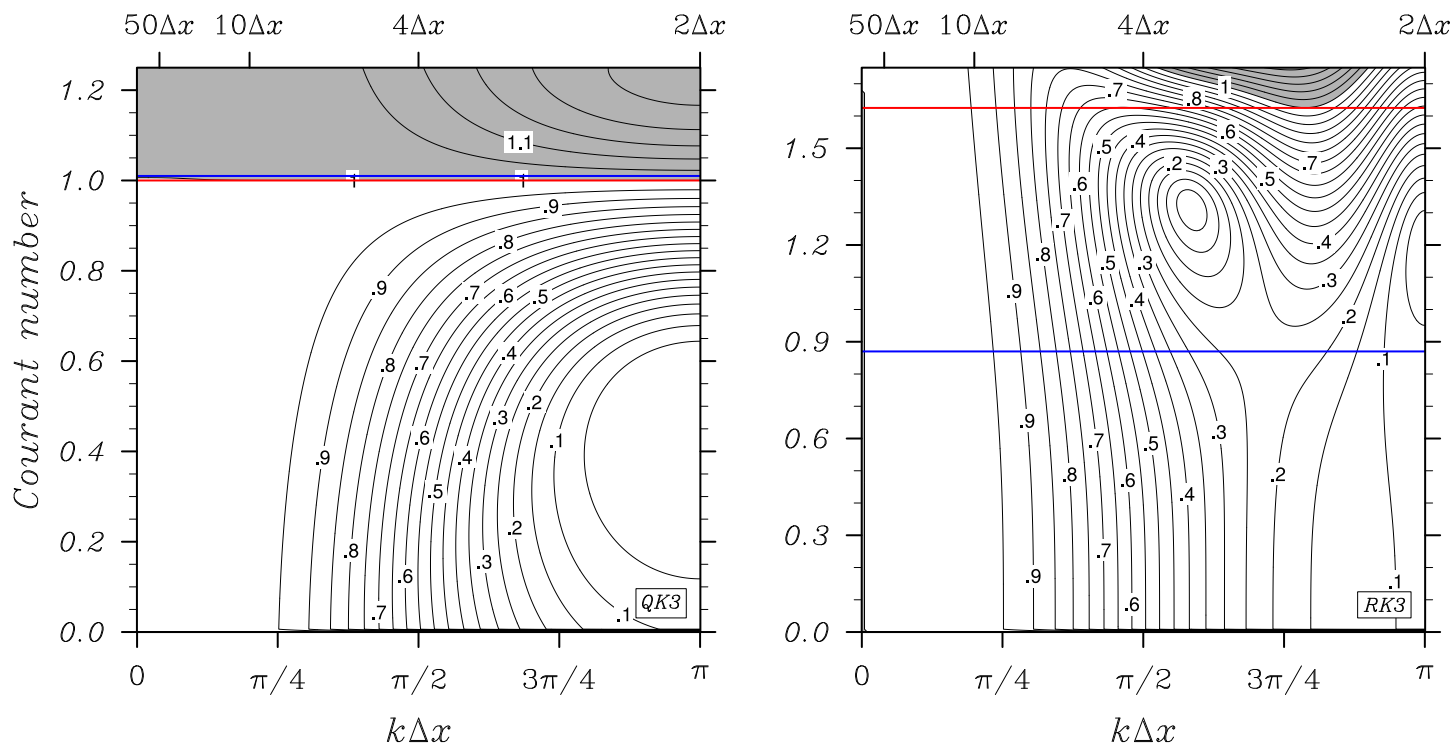

Fig. D.3. Same as Fig. D.2 for the physical mode of the third-order QK (Quickest) scheme (left) and RK3-UP3 (Runge-Kutta) scheme (right). Note the different vertical axis. 
does not always provide results consistent with the Fourier stability analysis. An example is the RK3-UP3 scheme which remains stable in the Von-Neumann sense between $\alpha_{\text {adv }}=1.25992$ and $\alpha_{\text {adv }}=1.626$ even for positive values of $\mathcal{G}$. In this range of Courant numbers, the leading order term of the truncation error exhibits an unstable solution exponentially growing with time, only stabilized by higher-order terms in the error. It can be shown that the behavior of this scheme over this range of Courant number is peculiar (cf Fig. D.3 and comments below). The AB3-UP3 scheme does not reach fourth-order accuracy on its stability range and becomes unstable even with a negative sign in front of the biharmonic leading order term (probably due to its computational mode).

\section{D.1.2 Robustness to changes in Courant number}

The results discussed in Sec. 3 suggest that an important property of a numerical scheme is its robustness to changes in Courant number. The derivative of $\mathcal{G}$ with respect to $\alpha_{\text {adv }}$ is representative of the evolution of the leading order term of the truncation error with respect to $\Delta t$, for a fixed $\Delta x$. It is straightforward to show that we get

$$
\frac{\mathrm{d} \mathcal{G}_{\mathrm{LFAM} 3}}{\mathrm{~d} \alpha_{\mathrm{adv}}}=\frac{7}{72}, \quad \frac{\mathrm{d} \mathcal{G}_{\mathrm{RK} 3}}{\mathrm{~d} \alpha_{\mathrm{adv}}}=\frac{\alpha_{\mathrm{adv}}^{2}}{8}, \quad \frac{\mathrm{d} \mathcal{G}_{\mathrm{QK} 3}}{\mathrm{~d} \alpha_{\mathrm{adv}}}=\frac{1+4 \alpha_{\mathrm{adv}}-3 \alpha_{\mathrm{adv}}^{2}}{24}, \quad \frac{\mathrm{d} \mathcal{G}_{\mathrm{AB} 3}}{\mathrm{~d} \alpha_{\mathrm{adv}}}=\frac{9 \alpha_{\mathrm{adv}}^{2}}{8}
$$

The evolution of $\mathrm{d} \mathcal{G} / \mathrm{d} \alpha_{\text {adv }}$ with respect to $\alpha_{\text {adv }}$ is plotted in Fig. D.1, right panel. From (D.2), we expect that the error associated with the LFAM3-UP3 scheme is not very sensitive to $\Delta t$ because the leading order term in the truncation error does not depend on $\alpha_{\text {adv }}$. For RK3-UP3 the error becomes independent of $\Delta t$ only for Courant numbers smaller than 0.1 (Fig. D.1). The behavior of the QK3 scheme is very different as the truncation error constantly changes with respect to $\Delta t$ which would make this scheme quite unpredictable in the context of complex flows with large spatial variations of the Courant number.

The previous remarks are consistent with the results shown in Fig. D.2 and D.3 where the amplitude error is plotted. In those figures, the absolute value of the physical mode $\left|\lambda^{\text {phys }}\right|^{\widetilde{\alpha} / \alpha_{\text {adv }}}$ (as well as the computational mode $\left|\lambda^{\text {num }}\right|^{\alpha / \alpha_{\text {adv }}}$ when relevant) with respect to the normalized wavenumber $k \Delta x$ and the Courant number $\alpha_{\text {adv }}$ is shown for the six space-time discretizations of interest with third-order upwind scheme in space. We plot $\left|\lambda^{\text {phys }}\right|^{\widetilde{\alpha} / \alpha_{\text {adv }}}$ to ensure that the amplification is compared on the same characteristic time interval whatever $\alpha_{\text {adv }}$. For all schemes the well resolved scales (i.e. for wavelength larger than $10 \Delta x$ ) are very well preserved but they sometimes coexist with a poorly damped computational mode (especially for the LFRA scheme, see left part of the region below the red line in Fig. D.2, f). For the LFAM3 scheme (Fig. D.2 a,d), the computational mode blows up first and is not always efficiently damped, especially for smaller scales ( $2 \Delta x$ and $4 \Delta x$ waves) with Courant number larger than 0.5. Unlike the AB3-UP3 scheme, stability of AB2 $\varepsilon$-UP3 is set by the physical mode and the computational mode is generally efficiently damped over the stability region (i.e. below the red line in Fig. D.2, e).

Let us now look at the behavior of the physical mode of the six discretization schemes (plotted in Fig. D.2 a,b,c and Fig. D.3) in their respective stability regions (i.e. below the red lines). Vertical contour lines in the $\left(\alpha_{\mathrm{adv}}, k \Delta x\right)$ plots would mean the absence of sensitivity of a given scheme to the Courant number. As expected from our analysis of the truncation error, the LFAM3-UP3 scheme (at a lesser degree the AB3-UP3 scheme) shows very modest sensitivity, except for the $2 \Delta x$ waves (Fig. D.2, a). The AB2 $\varepsilon$-UP3 scheme shows nearly horizontal contour lines for Courant numbers in the range $0.4-0.57$ indicating large amplification error changes with respect to $\alpha_{\text {adv }}$ (Fig. D.2, b). For Courant numbers smaller than 0.4 the 
scheme is significantly more robust. For the LFRA-UP3 scheme the contour lines are increasingly bent with increasing wavenumbers and become nearly horizontal for $2 \Delta x$ waves. For example, the amplification factor of the $4 \Delta x$ waves varies between 0.45 and 0.7 which is quite substantial (Fig. D.2, c). As expected from earlier discussion, the amplification error of the QK3 and RK3-UP3 schemes show large sensitivity to the Courant number for scales smaller than $10 \Delta x$ (Fig. D.3). Because the QK3 scheme is exact for $\alpha_{\mathrm{adv}}=1$, it is not surprising to see that the amplification error varies quickly when decreasing the Courant number. However, those changes occur predominantly between $k \Delta x=\pi / 2$ (i.e. $4 \Delta x$ waves) and $k \Delta x=\pi$ (i.e. $2 \Delta x$ waves) whereas the amplitude of waves larger than $4 \Delta x$ is remarkably well preserved. The amplification factor for $4 \Delta x$ waves varies between 0.7 and 1 (Fig. D.3, a). Finally, the RK3-UP3 scheme exhibits two subregions (delimited by the line $\alpha_{\mathrm{adv}}=0.8$ ) with very different behavior. For $\alpha_{\mathrm{adv}} \leq 0.8$ the scheme behaves well. For Courant numbers above 0.8 (which includes the region where the leading order term of the truncation error is a biharmonic with positive coefficient) the amplification factor shows very unpredictable behavior for $k \Delta x \geq 3 \pi / 8$. A drawback of the QK3 and RK3-UP3 schemes is that for certain Courant numbers they can leave the $2 \Delta x$ waves poorly damped.

\section{D.1.3 Monotonic damping}

An additional constraint besides the Von-Neumann stability condition $(|\mathcal{A}| \leq 1$, with $\mathcal{A}$ the amplification factor of a given space-time discretization) would be to consider that an odd-ordered numerical scheme should also satisfy a monotonic damping property, $\partial|\mathcal{A}| / \partial k<0$, for $|k| \in[0, \pi / \Delta x]$ (i.e. smaller scales are always more efficiently damped than larger scales). This property is satisfied by the exact diffusion operator. Failing to satisfy this property means that energy spectrum may no longer monotonically decrease with respect to wavenumbers and that energy could accumulate at intermediate scales. For the six schemes under consideration, monotonic damping is satisfied in the region below the blue lines in the $\left(\alpha_{\text {adv }}, k \Delta x\right)$ frame plotted in Fig. D.2, D.3. When $|\mathcal{A}| \leq 1$ (i.e. when the scheme is stable in the VonNeumann sense), the QK3 scheme satisfies the desired property. However, this is not the case for other schemes : the RK3-UP3 scheme damps scales monotonically only for $\alpha_{\text {adv }} \leq 0.87$ (compared to the CFL number $\left.\alpha_{\mathrm{adv}}^{\star}=1.626\right)$, for $\alpha_{\mathrm{adv}} \leq 0.375$ for the LFRA-UP3 scheme ( $\left.\alpha_{\mathrm{adv}}^{\star}=0.472\right), \alpha_{\mathrm{adv}} \leq 0.425$ for the AB2-UP3 scheme $\left(\alpha_{\mathrm{adv}}^{\star}=0.576\right), \alpha_{\mathrm{adv}} \leq 0.3$ for the AB3-UP3 scheme $\left(\alpha_{\mathrm{adv}}^{\star}=0.394\right)$ and $\alpha_{\mathrm{adv}} \leq 0.59$ for LFAM3-UP3 ( $\alpha_{\text {adv }}^{\star}=0.871$ ). While the lack of monotonic damping affects only the $2 \Delta x$ waves for LFAM3, AB2 and LFRA, it also affects larger scales in the case of the RK3.

\section{D.2 Group Velocity Analysis}

In this section, we analyze the group velocity error associated with various space-time discretizations of the linear advection equations (in this case the analytical group speed is the advection speed $u_{0}$ ). The group velocity corresponds to the speed at which the energy of a wave will travel. We emphasize the fact that several features that are not apparent from the phase speed relations can be seen by examining the group velocities. We study the six different time-schemes previously introduced along with a third-order accurate scheme in space. It is generally considered that a fourth-order centered scheme should provide the same group velocity errors of a third-order scheme. This statement is, however, only partially true because when using a dissipative advection scheme (and/or a dissipative time-integration scheme), some scales (high-frequency waves, in general) are efficiently damped and are no longer present in the solution. Those scales should not be considered in the group velocity analysis (e.g. Karni, 1994).

Without further details (see Durran, 2010, for a thorough description), we define the amplification 

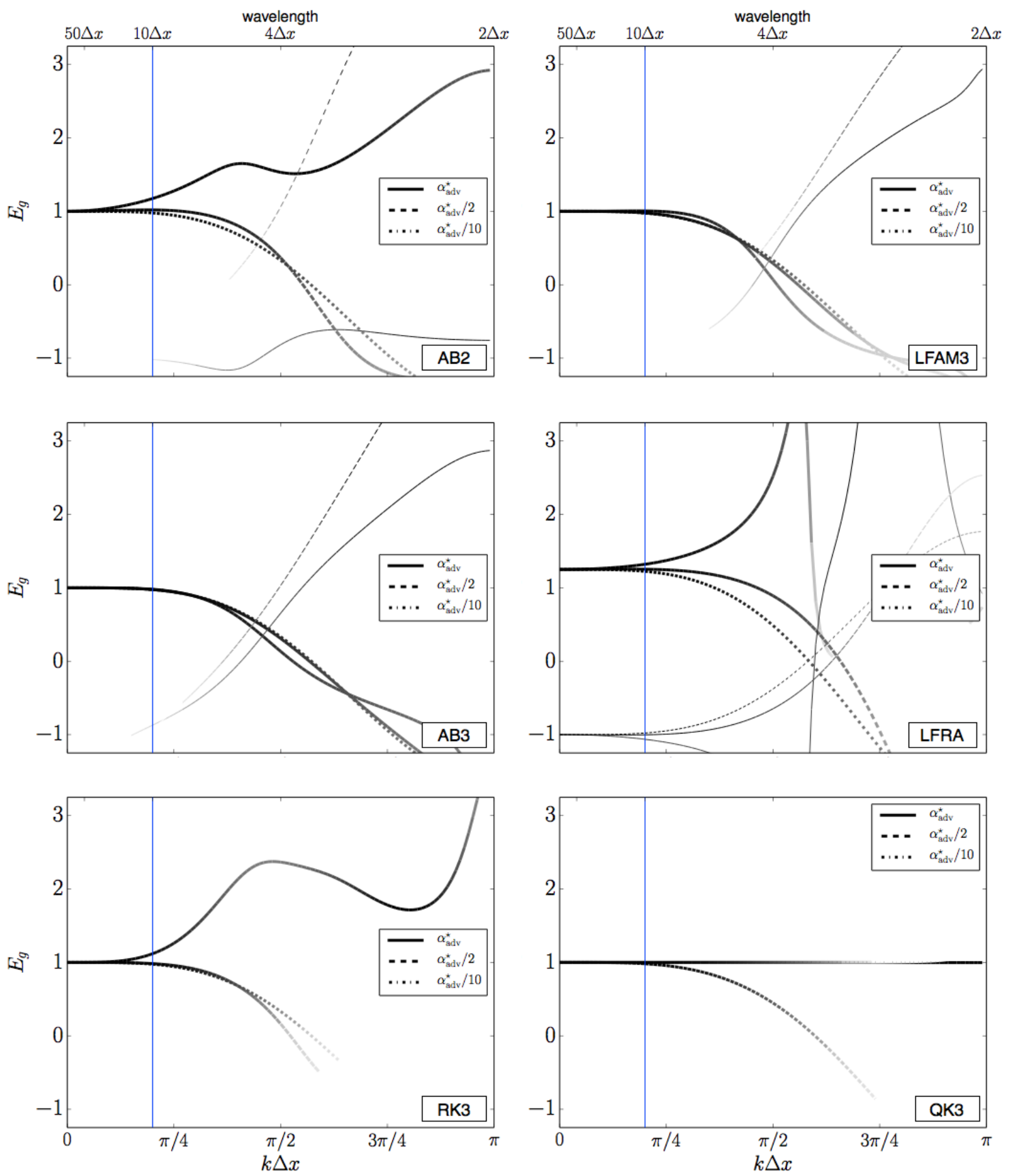

Fig. D.4. Evolution of the Group velocity error $E_{g}$ with respect to the normalized wavenumber $k \Delta x$ for six different space-time discretizations. Results are shown for the physical mode (thick lines) and for the computational mode (thin lines), when present. Solid lines are for $\alpha_{\mathrm{adv}}=\alpha_{\mathrm{adv}}^{\star}$ (i.e. at the stability limit), dashed lines for $\alpha_{\mathrm{adv}}=\alpha_{\mathrm{adv}}^{\star} / 2$, and dot-dashed lines for $\alpha_{\mathrm{adv}}=\alpha_{\mathrm{adv}}^{\star} / 10$. Each curve of $E_{g}$ is gray scaled from black when the scale is not damped (i.e. the associated amplification factor is close to 1 ) to white when the scale is efficiently damped by the numerical scheme (i.e. the associated amplification factor is close to 0 ). The top $\mathrm{x}$-axis represents wavelengths. The vertical blue line denotes a separation between scales larger than $10 \Delta x$ (on the left) and smaller scales (on the right). The acronyms are LFAM = Leapfrog Adams-Moulton, LFRA = Leapfrog Robert-Asselin, AB=Adams-Bashforth, RK = Runge-Kutta, QK= Quickest. 
factor $\mathcal{A}=\left|\lambda^{\text {phys }}\right|$, with $\lambda^{\text {phys }}$ a root of the characteristic equation, and the group velocity error as

$$
E_{g}=\frac{v_{g}}{u_{0}}, \quad v_{g}=\frac{\Delta x}{\Delta t} \frac{\partial}{\partial \theta}\left(\arctan \left(\frac{\Im\left(\lambda^{\text {phys }}\right)}{\Re\left(\lambda^{\text {phys }}\right)}\right)\right),
$$

with $\theta=k \Delta x$ the normalized Fourier frequency in the $x$-direction $(|\theta| \leq \pi)$. When $E_{g}$ is close to 1 the group velocity is accurately estimated and when it is negative the group speed is in the wrong direction. Formula (D.3) also applies to the computational mode $\lambda^{\text {num }}$. Fig. D.4 shows $E_{g}\left(\lambda^{\text {phys }}\right)$ (as well as $E_{g}\left(\lambda^{\text {num }}\right)$, when relevant) with respect to $k \Delta x$. Moreover, each curve of $E_{g}$ is gray scaled from black when the scale is not damped to white when the scale is efficiently damped by the numerical scheme. Results are shown for different Courant numbers : $\alpha_{\mathrm{adv}}^{\star}, \alpha_{\mathrm{adv}}^{\star} / 2$ and $\alpha_{\mathrm{adv}}^{\star} / 10$.

The well-resolved scales, i.e. for wavenumbers larger than $10 \Delta x$ (left of the blue lines in Fig. D.4), are very well represented by the QK3, AB3-UP3 and LFAM3-UP3 schemes, whatever the Courant number. The AB2-UP3 and RK3-UP3 tend to predict too fast of a group velocity for those scales close to the stability limit. The LFRA-UP3 scheme exhibits large errors because this is the only scheme for which the computational mode is not efficiently damped for larger scales. As far as smaller scales are concerned (i.e. for wavenumbers smaller than $10 \Delta x$ ), almost all the schemes predict a stationary state (i.e. $E_{g} \approx 0$ ) for $4 \Delta x$ wave packets. More generally, the group velocity of high-frequency modes is shown to assume the incorrect sign $\left(E_{g} \leq 0\right)$, thus admitting backward propagation of waves which persist without being dissipated. The RK3-UP3 and QK3 schemes damp efficiently the waves for which the group velocity is in the wrong direction (i.e. the curves turn white when there is a significant derparture from $E_{g}=1$ ), except close to its stability limit for RK3-UP3. The LFAM3-UP3 and AB3-UP3 schemes have less damping, and the computational mode can become dominant for $2 \Delta x$ waves (i.e. thin lines are darker than the thick lines for $k \Delta x \geq 3 \pi / 4$ ). Again, the LFRA-UP3 scheme is difficult to interpret because of the coexistence of two competing modes.

In general, the group velocity analysis is clean and easy to interpret for schemes free of computational modes. In the presence of such modes it is difficult to interpret the results for small scales because the physical and computational mode generally have comparable damping rates. 